\title{
ALTERNATIVE METHODOLOGIES TO THE SEMI-ANALITIC SOLUTION TO THE PROBLEM OF MOVING LOADS ON HIGH SPEED RAILWAY BRIDGES BASED ON THE SENSITIVITY OF THE RESPONSE TO TRAIN SPEED AND ON HILBERT TRANSFORMATION.
}

MASTER'S DEGREE FINAL PROJECT

Por

Enrique García Macías.

I.C.C.P.

Tutor

Alejandro Martínez Castro

Department of Structural Mechanics and Hydraulic Engineering ,

University of Granada

Edificio Politécnico Fuentenueva, C/ Severo Ochoa s/n, CP 18002

Granada (España)

September 2013 

(C) Copyright 2013

por
Enrique García Macías. 



\begin{abstract}
Nowadays, modernity and high speed rail are terms inevitably coupled. The bad experience in the predominance of the transportation by the road over the rest of ways of transportation makes societies ask for new sustainable and efficient way of transportation, role for which high speed rail is positioned to be one of the most important pieces. Because of this, Engineering must be prepared to plan these new structures and face the design of them according to the sensitive social conscience about something as important as the transportation. From the point of view of the design, high speed bridges are structures whose dynamic response conditions the while design process. Nevertheless, despite the more than foreseeable increase demand on these structures, the dynamic analysis techniques keep on using approximate techniques based on step by step methodologies, methods that discretize as much time as space. Semi-analytic solution supposes a great improvement on dynamic analysis of high speed bridges subjected to moving load because it only discretizes space but it is exact on the time domain. However, despite being a more accurate and fast methodology in the analysis, today it still requires great amount of time of calculus and computational costs in the analysis of great tridimensional structures. This fact is more critical in design process in which the engineer must deliberate between several alternatives for the final design, having to employ great amounts of time in each one of them. The present research proposes two new methodologies based on Semi-Analytic solution but optimized in the two main variables of the analysis, time and velocity stepping, thanks to its analytical properties in the time domain what allows a deep mathematical treatment of the formulation. On one hand, the first proposed method is called the $\mathrm{dq} / \mathrm{dv}$ method, and it defines the response of the structure through the determination of its sensibility to velocity what allows higher velocity sampling. On the other hand, it is defined a new methodology through the application of the Hilbert transformation of the signals what allows a higher time samplig, the other main variable. The relevance of this two new methodologies lays on their applicability in pre-design phases, in which the reduction of computational cost and time of calculus can be very important.
\end{abstract}





\section{Acknowledgment}

First of all, I would like to express my enormous gratitude to my father, Jorge, for the permanent and unconditional support and his good advices that have always gone with me from the very beginning of my degree and until the end of this last step, my post-graduate degree in Structures. I neither could lose this chance to show my deep gratitude for my lovely mother, Concepción, for building, aside my father, a beautiful home in which i have grown up and $i$ have had the chance of choosing my way inside Civil Engineering.

I would also express my eternal gratitude to Prof. Alejandro Martinez Castro, for his patience and commitment not only to professional but also personal. I met him at the University of Granada in 2007 from the role of student, then with the realization of my final degree project and today with this post graduate degree project. As for this project, their support has been constant, from providing a platform for intensive computation, notions about signal conditioning, ideas about computational optimization...etc, but above all, i am actually pleased about have been able to meet the way he understands Engineering, not only a powerful tool to make practical projects come true but also a rightful field to make Science.

Secondly i would like to give special thanks to Prof. Rafael Gallego as the coordinator of the Master of Structures of the University of Granada.

Finally, I would like to express my eternal gratitude to my colleagues and friends with whom i have shared this beautiful and last step until the beginning of a hopeful professional career. 
Dedicated to my lovely parents, Jorge and Concepción. 


\section{Contents}

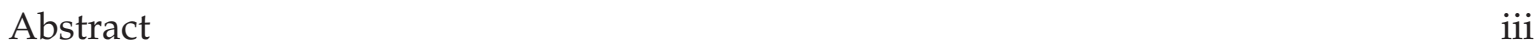

Acknowledgment $\quad$ v

Chapter 1 Introduction, objectives and methodology 1

1.1 Introduction . . . . . . . . . . . . . . . . . . . . 1

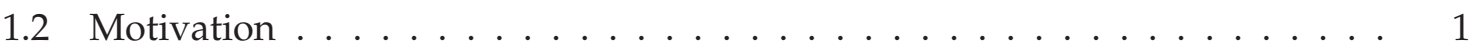

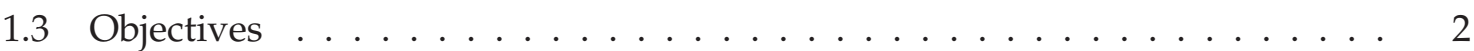

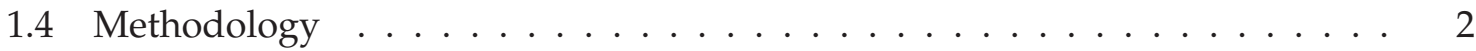

1.5 Literature Review . . . . . . . . . . . . . . . . . . . . . . 2

1.5.1 Dynamic solutions to the response of bridge under moving loads . . . 2

1.5.2 Hilbert's transformation and its applications to engineering . . . . . 3

Chapter 2 Method 1: dq/dv. Derivate of the maximum acceleration respect to the train velocity 5

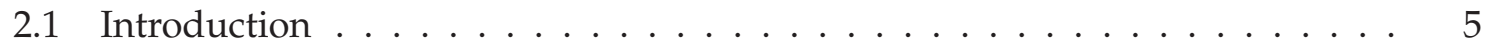

2.2 Theoretical development . . . . . . . . . . . . . . 6

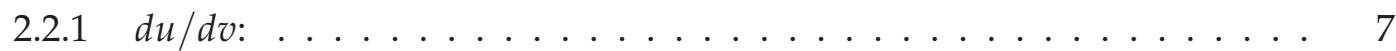

$2.2 .2 d \ddot{u} / d v: \ldots \ldots \ldots \ldots \ldots \ldots \ldots \ldots \ldots \ldots$

2.2.3 Applicability to maximum envelope determination . . . . . . . . . 9

2.3 Test . . . . . . . . . . . . . . . . . . . . . . . 14

2.3.1 Simply supported beam under a single moving load (IAPF) . . . . . . 14

2.3.2 A three-span continuous stepped beam under a single moving load. (Henchi). . . . . . . . . . . . . . . . . . . . . 19

2.3.3 Three-span bridge according to Eurocode $1 . \ldots \ldots 23$

2.3.4 Bowstring steel-concrete composite bridge . . . . . . . . . . . . 28

2.3.5 Concrete box girder bridge. Rodenillo bridge. . . . . . . . . . . . . . 33

2.3.6 Steel-concrete composite bridge. Sesia viaduct. . . . . . . . . . . . . . . 46

Chapter 3 Method 2: Hilbert's transformation. 59

3.0 .7 Introduction . . . . . . . . . . . . . . . . . . . . 59

3.0.8 Application to Semi-Analytic methodology . . . . . . . . . . . . . 61

3.0 .9 Validation . . . . . . . . . . . . . . . . . . . . 68

3.0.10 Window filter . . . . . . . . . . . . . . . . 68 
3.0.11 Validation with numeric approximations . . . . . . . . . . 68

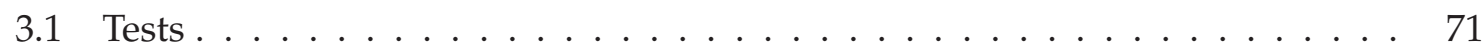

3.1.1 Simply supported beam (IAPF) . . . . . . . . . . . . . . . . 71

3.1.2 Effect of increasing time sampling . . . . . . . . . . . 76

3.1.3 Concrete box girder bridge. Rodenillo Bridge. . . . . . . . . . . . . . . 80

3.1.4 Steel-concrete composite bridge. Sesia viaduct. . . . . . . . . . . . . . . 81

Chapter 4 Conclusions and future improvement 85

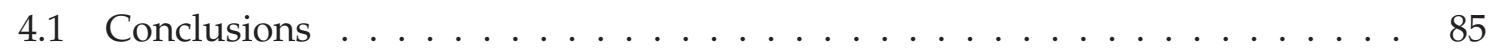

4.2 Future improvement $\ldots \ldots \ldots \ldots \ldots \ldots$ 


\section{List of Tables}

2.1 One single load central differences analysis $\ldots \ldots \ldots \ldots$. . . . . . . . . . 21

2.2 Two loads central differences analysis. . . . . . . . . . . . . . . . . 22

2.3 Modes and frequencies used in three-span brdige according to Eurocode 1. . $\quad 23$

2.4 Masses and properties of elements employed in U-Girder modelç . . . . . . . 34

2.5 Fixed damping ratios in Rayleigh's damping method in Rodenillo . . . . . . . 38

2.6 Main materiales in the Sesia's FEM model. . . . . . . . . . . . . . . . . . . 50

2.7 Natural frequencies of Sesia viaduct. . . . . . . . . . . . . . . . . . . . . . 50

2.8 Fixed damping ratios in Rayleigh's damping method in Sesia viaduct. . . . . 52 



\section{List of Figures}

2.1 Obtaining of one point of maximum envelope curve in dynamic analysis. . . 5

2.2 Obtaining of maximum envelope curve in dynamic analysis. . . . . . . . . . . 6

2.3 Scheme of local envelope curve of response in dynamic analysis. . . . . . . . 13

2.4 Local response surface of structure under moving loads. . . . . . . . . . . . . 14

2.5 Simply supported beam . . . . . . . . . . . . . . . . . . . 15

2.6 Semianadin vs Analytical . . . . . . . . . . . . . . . . . 17

2.7 Semianadin vs Central difference derivatives . . . . . . . . . . . . . . . . . 18

2.8 Semianadin vs Analytical . . . . . . . . . . . . . . . . . . 18

2.9 Semianadin vs Central difference derivatives . . . . . . . . . . . . . . . . . . . 19

2.10 A three-span continuous stepped beam . . . . . . . . . . . . . . . . . 19

2.11 Max-Min Envelopes of test 2 . . . . . . . . . . . . . . . . . 20

2.12 Detail of maximum acceleration test $2 \ldots \ldots \ldots$. . . . . . . . . . 20

2.13 Acceleration envelope and analyzed velocities . . . . . . . . . . . . 22

2.14 Load case 1: Two point loads. . . . . . . . . . . . . . . . . . . . 22

2.15 Acceleration envelope and analyzed velocities . . . . . . . . . . . . . . . . 23

2.16 Three-span bridge according to Eurocode 1 . . . . . . . . . . . . . . . . 23

2.17 Acceleration Envelope. Train $A_{1} \ldots \ldots \ldots \ldots$. . . . . . . . 24

2.18 Acceleration Envelope. All trains. . . . . . . . . . . . . . . . . . 25

2.19 Maximum Absolute Acceleration with velocity steps 10 and $20 \mathrm{~km} / \mathrm{h}$ per $\mathrm{km} / \mathrm{h} 26$

2.20 Relative Errors in Maximum acceleration. . . . . . . . . . . . . . . . . . . 27

2.21 Relative errors associated to two different velocity steps, 10 and $20 \mathrm{~km} / \mathrm{h}$ per

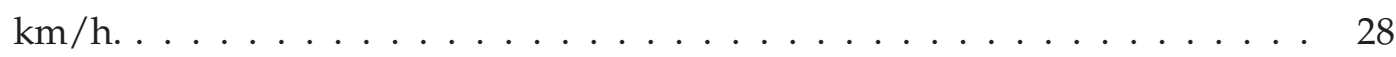

2.22 Elevation view. . . . . . . . . . . . . . . . . . . . . . . . . . . . . 29

2.23 Cross section of lateral beams. . . . . . . . . . . . . . . . . . . . . . 29

2.24 Tridimensional FEM. . . . . . . . . . . . . . . . . . . . . . 30

2.25 Detail of lateral beams and the joint with the concrete deck. . . . . . . . . . . 31

2.26 Transversal variable height beams detail. . . . . . . . . . . . . . . . . 31

2.27 Acceleration envelope in mid central span under train A1 of HSML series . 32

2.28 Displacement envelope in mid central span under train A1 of HSML series . 32

2.29 Lateral view. . . . . . . . . . . . . . . . . . . . . . . . . . . . 33

2.30 Cross section . . . . . . . . . . . . . . . . . . 34

2.31 FEM model . . . . . . . . . . . . . . . . . . . . . . . . 35

2.32 FEM 3D model . . . . . . . . . . . . . . . . . . . . . . . 35 
2.33 Natural modes. (a) first bending mode $2.58 \mathrm{~Hz}$, (b) second bending mode

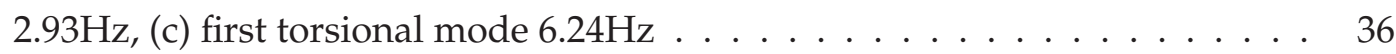

2.34 Post-process points and load line scheme. . . . . . . . . . . . . . . 36

2.35 Train A1, post-process point 1. Effects of velocity stepping. . . . . . . . . . . 37

2.36 Train A1, post-process point 1. Effect of time stepping. . . . . . . . . . . . . 38

2.37 Train A1, post-process point 1. Acceleration under Rayleigh damping. . . . . 39

2.38 Time serie for train A1, $150 \mathrm{~km} / \mathrm{h}$ and constant modal damping ratio of $2 \% \quad$. $\quad 40$

2.39 Time serie for train A1, 150km/h and Rayleigh's damping . . . . . . . . . 40

2.40 Time serie for train $A 1,325 \mathrm{~km} / \mathrm{h}$ and constant modal damping ratio of $2 \%$. . 40

2.41 Time serie for train A1, 325km/h and Rayleigh's damping . . . . . . . . . . 41

2.42 Acceleration Envelope, point 1. . . . . . . . . . . . . . . . . 41

2.43 Relative error on acceleration envelopes of point 1 . . . . . . . . . . . . . 42

2.44 Train A1, post-process point 1. Effect of velocity stepping. . . . . . . . . . . 43

2.45 Train A1, post-process 1. Effect of time stepping. . . . . . . . . . . . . . . 44

2.46 Train A1, post-process 1. Displacement evaluation under Rayleigh damping. 44

2.47 Displacement envelope on post-process point $1 . \ldots \ldots$. . . . . . . . 45

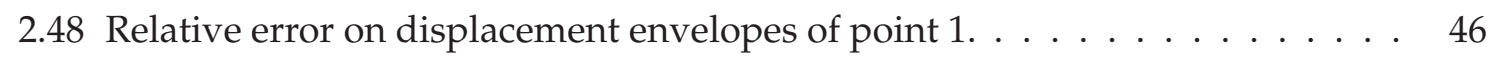

2.49 Elevation and front view of Sesia. . . . . . . . . . . . . . . . . . . . . . . . . . . . . . . . . .

2.50 Cross sections of viaduct of Sesia. . . . . . . . . . . . . . . . 47

2.51 Boundary conditions on Sesia viaduct. . . . . . . . . . . . . . . . . 49

2.52 3D FEM model of Viaduct sesia. . . . . . . . . . . . . . . . . . . . . . . 49

2.53 Natural modes of the Sesia viaduct. . . . . . . . . . . . . . . . . 50

2.54 Train A1, post-process point 5 . Effects of velocity stepping. . . . . . . . . . . 51

2.55 Train A1, post-process point 5. Effects of time stepping. . . . . . . . . . . . . 52

2.56 Train A1, post-process point 5. Acceleration evaluation under Rayleigh damp-

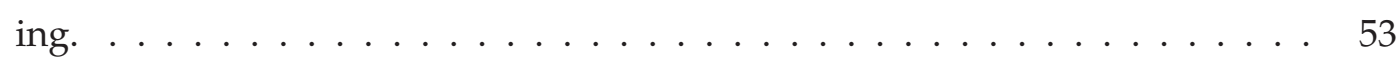

2.57 Acceleration envelope on post-process point $5 . \ldots \ldots \ldots$. . . . . . . 54

2.58 Relative error on acceleration envelopes of point $5 . \ldots$. . . . . . . . . . . 54

2.59 Train A1, post-process point 2. Effects of velocity stepping. . . . . . . . . . . 55

2.60 Train A1, post-process point 2. Effects of time stepping. . . . . . . . . . . . . 56

2.61 Train A1, post-process point 2. Displacement evaluation under Rayleigh damp-

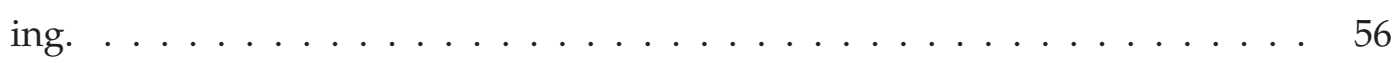

2.62 Displacement envelope on post-process point $2 \ldots \ldots$. . . . . . . . 57

2.63 Relative error on displacement envelopes of point 2 . . . . . . . . . . . . 57

3.1 (a)Impulse response function of the ideal HT; (b)Module of the HT transfer function; (c) Phase of the HT transfer function. . . . . . . . . . . . . . . . . . 62

3.2 ExpIntegralEi function . . . . . . . . . . . . . . . . . 63

3.3 Complete Hilbert transformation of the homogeneous part of Semi-Analitic solution. . . . . . . . . . . . . . . . . . . 64

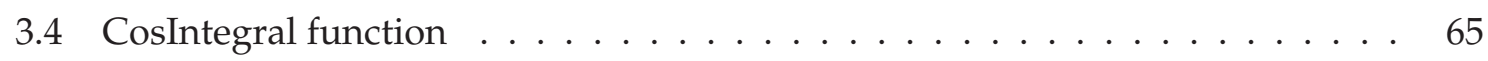


3.5 SinIntegral function . . . . . . . . . . . . . . . .

3.6 Hilbert transformation of the homogeneous part of Semi-Analitic solution based on the product theorem. . . . . . . . . . . . . . . . 66

3.7 Local Hilbert transformation of the homogeneous Semi-Analitic solution. . . 67

3.8 Acceleration time serie by complete Hilbert, window filter and numerical transformation of the eurocode 1 train under train A1 of HSML series and

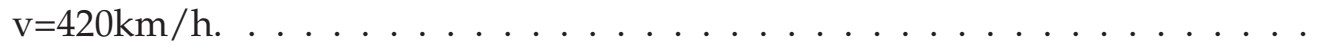

3.9 Detail of acceleration time serie by complete Hilbert, window filter and numerical transformation of the eurocode 1 train under train A1 of HSML series

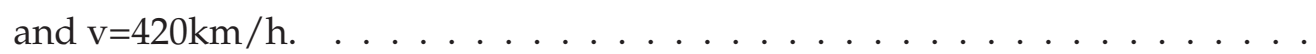

3.11 Detail of displacement time serie by complete Hilbert, window filter and numerical transformation of the eurocode 1 train under train A1 of HSML series

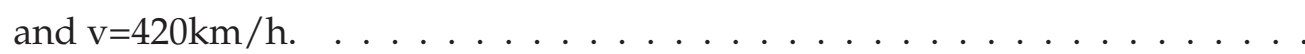

3.10 Displacement time serie by complete Hilbert, window filter and numerical transformation of the eurocode 1 train under train A1 of HSML series and

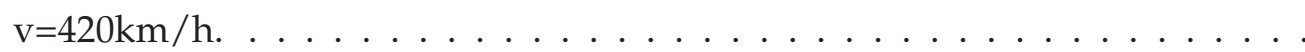

3.12 Semi-Analytic time series of acceleration and its complete Hilbert's transformation of mid span of simply supported beam (IAPF) under a single load

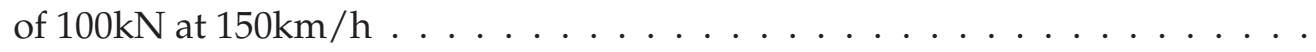

3.13 Semi-Analytic ime series of displacement and its complete Hilbert's transformation of mid span of simply supported beam (IAPF) under a single load of $100 \mathrm{kN}$ at $150 \mathrm{~km} / \mathrm{h} \ldots \ldots \ldots \ldots$. . . . . . . . . . . . . . .

3.14 Acceleration envelope of mid span of simply supported beam (IAPF) under a single load of $100 \mathrm{kN} \ldots \ldots \ldots \ldots \ldots$

3.15 Displacement envelope of mid span of simply supported beam (IAPF) under a single load of $100 \mathrm{kN} \ldots \ldots \ldots \ldots \ldots \ldots \ldots \ldots \ldots \ldots \ldots \ldots$

3.16 Time series of acceleration of mid span of simply supported beam (IAPF) under the train A1 of the HSML series at $500 \mathrm{~km} / \mathrm{h} \ldots \ldots$. . . . . . . . . 74

3.17 Time series of displacement of mid span of simply supported beam (IAPF) under the train $\mathrm{A} 1 \mathrm{of}$ the HSML series at $500 \mathrm{~km} / \mathrm{h} \ldots \ldots \ldots$. . . . . . .

3.18 Time series of acceleration of mid span of simply supported beam (IAPF)under the train ICE2 at $150 \mathrm{~km} / \mathrm{h} \ldots \ldots \ldots \ldots \ldots$

3.19 Acceleration time series of mid span of simply supported beam (IAPF) under a single load of $100 \mathrm{kN}$ at $150 \mathrm{~km} / \mathrm{h}$ obtained by Semi-Analytic solution, complete Hilbert transformation and Hilbert transformation with window filter of $10 \mathrm{~m} \ldots \ldots \ldots \ldots \ldots \ldots \ldots \ldots \ldots \ldots \ldots \ldots \ldots \ldots \ldots \ldots$

3.20 Acceleration envelope of mid central span of Henchi beam under a single load of $9.8 \mathrm{kN}$ by Semi-Analytic solution and Hilbert transformation for increasing

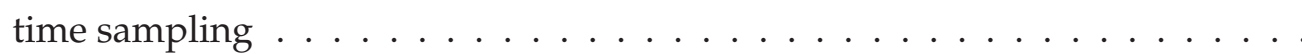


3.21 Displacement envelope of mid central span of Henchi beam under a single load of $9.8 \mathrm{kN}$ by Semi-Analytic solution and Hilbert transformation for increasing time sampling . . . . . . . . . . . . . . . . . 77

3.22 Acceleration envelope of mid span of Simply supported beam (IAPF) under a two loads of $9.8 \mathrm{kN}$ by Semi-Analytic solution and Hilbert transformation for increasing time sampling . . . . . . . . . . . . . .

3.23 Displacement envelope of mid span of Simply supported beam (IAPF) under a two loads of $9.8 \mathrm{kN}$ by Semi-Analytic solution and Hilbert transformation for increasing time sampling . . . . . . . . . . . . . . . . 78

3.24 Acceleration envelope of mid span of Three-span bridge according to Eurocode 1 under the trains of HSML series by Semi-Analytic solution and Hilbert transformation for increasing time sampling . . . . . . . . . . . . . . 79

3.25 Displacement envelope of mid span of Three-span bridge according to Eurocode 1 under the trains of HSML series by Semi-Analytic solution and Hilbert transformation for increasing time sampling . . . . . . . . . .

3.26 Acceleration envelope of concrete box girder bridge subjected to the passage of train A1 of HSML series by Semi-Analytic method and by $20 \mathrm{~m}$ windowfiltered Hilbert envelope with increasing time sampling. . . . . . . . . . . .

3.27 Displacement envelope of concrete box girder bridge subjected to the passage of train A1 of HSML series by Semi-Analytic method and by $20 \mathrm{~m}$ windowfiltered Hilbert envelope with increasing time sampling. . . . . . . . . . . . 81

3.28 Acceleration envelope of steel-concrete composite bridge subjected to the passage of train A1 of HSML series by Semi-Analytic method and by $20 \mathrm{~m}$ windowfiltered Hilbert envelope with increasing time sampling. . . . . . . . . . . .

3.29 Displacement envelope of steel-concrete composite bridge subjected to the passage of train A1 of HSML series by Semi-Analytic method and by $20 \mathrm{~m}$ window-filtered Hilbert envelope with increasing time sampling. . . . . . . . 


\section{CHAPTER 1}

\section{Introduction, objectives and methodology}

\subsection{Introduction}

The final aim of the dynamic analysis of mobile loads over a bridge is, mainly, the obtaining of an acceleration or displacement envelope curve which matches its maximum values with the train velocity. In order to obtain these curves it is necessary to determine all the time series for all the trains and train velocities, as well as a posterior search of the maximum values. Because of this, as an example, the dynamic analysis of 12 trains (10 from the HSML series, AVE and TALGO), from $20 \mathrm{~km} / \mathrm{h}$ to $270 \mathrm{~km} / \mathrm{h}\left(1.2 \cdot V_{\max }\right)$ with velocity steps of $10 \mathrm{~km} / \mathrm{h}$, the maximum allowable velocity steps established by the standard IAPF-07, would require the determination of 300 time series and a posterior maximum detection. Nevertheless, sampling of envelopes with velocity steps of $10 \mathrm{~km} / \mathrm{h}$ is usually not enough to register correctly the resonant effects, that is why it is convenient to set a sampling with steps of $1 \mathrm{~km} / \mathrm{h}$, this, the number of direct calculations is increased to 2000 time series. Because of this, it is not difficult to realize the most important problem that these analysis must face is the huge computational cost and the times of calculus required to obtain the results. Traditionally, direct integration of the differential equation of movement with moving loads was carried out by the called step-by-step method, above all, by the Newmark-Beta method. These methods discretize both dimensions of the problem, time and space variables. However, in [1], it is published an alternative by A. M. Castro et al, the Semi-Analitic solution. This methodology overtakes the classical methods by obtaining the exact solution of the time part of the differential motion, after a variable separation through modal analysis. Because of this, the solution is obtained much faster because the problem is reduced to the evaluation of an analytical solution and, because the time dimension is not discretized, the precision of the results are finer than the ones obtained by step-by-step methods.

\subsection{Motivation}

Dynamic analysis to moving loads on bridges has two main variables that control all the process: time and velocity. All this process can be divided into two parts: time series determination and maximum envelope construction. On one hand, time stepping determine the precision of the time series of a train going through a bridge with a determined velocity. on the other hand, the final target of all this process is to obtain the maximum acceleration/displacement envelope curve. This curve involves all the registered maximum values in the response of the structure under each train velocity. On one hand, time controls the 
precision of the time series, as a result, it also controls the precision of the later determination of the maximum value. On the other hand, velocity steps of the trains, in which the response of the structure are determined, control the accuracy of the envelope curves. SemiAnalitic solution offers a perfect field to employ analytic optimization strategies because the solution is analytic in the time dimension.

Over the basis of Semi-Analytical solution, in the present document, two different methodologies are proposed to improve the dynamic analysis in terms of computational costs and time of calculus. The first one, $\mathrm{dq} / \mathrm{dv}$, attacks velocity variable. This methodology employs higher velocity steps and finally, to define a correct maximum envelope and not neglecting the possible resonant effects, this methodology fits a cubic polynomial expression between each two consecutives points interpolating the maximum value of the analyzed variable as well as its sensitivity to velocity. On the other hand, the second methodology attacks time variable by the application of a Hilbert's transformation of the signals. This transformation determines the envelope of the time series, much smoother than original ones so it is possible to sample this new signal with higher time steps.

\subsection{Objectives}

- Analysis of the state of the art in order to point out the deficiencies of the current practical methodologies.

- Development of alternative methodologies in order to solve some of the problems found in this field.

- Validation of the methodologies via finite difference derivation and by the application to real cases.

- Proposal and development of two new faster direct integration methodologies to the problem of moving loads on bridges.

\subsection{Methodology}

- Analysis of the state of the art

- Theoretical development of the proposed methodologies: dq/dv and Hilbert's transformation.

- Validation of the methodologies.

- Conclusions

\subsection{Literature Review}

\subsubsection{Dynamic solutions to the response of bridge under moving loads}

In the engineering field, the dynamic problem of the passage of moving loads has a high interest in the field of design of railway bridges and highway bridges. This problem consists on the idealization of a vehicle as a set of concentrated loads moving over a load line with constant velocity. In the methods recommended by any standard treat the dynamic analysis through approximate solutions and being able to obtain the exact solutions in just few certain cases, such as in the work of Bleich [2] and Kryloff [3] for isostatic structures 
(also see the works shown in the books of Fryba [4], Biggs [5] or Yang et al [6]), for very specific exponential loads as the work of Chen and Li [7] or for staggered edge beams as the work of Hayashikawa and Watanabe [8]. For a general load on a generic structure, the technique uses integration methods step by step, "Step -by -Step " (see the book of Clough and Penzien [9]), and particularly, as the most common method, Newmark-Beta [10]. In reference to the modelization in the space domain with finite element method, it can be pointed out the works of Calçada et al [11], Kim et al. [12], Kwark et al. [13] and Karoumi [14] in its application to cable-stayed bridges. Finally, among the numerous studies that consider dynamic interation vehicle-structure, the work of Majka and Hartnett [15] presents a study in which they address vehicle-structure interaction in high speed bridge with a NewmarkBeta direct integration combined with an iterative Newton-Raphson method and a solution in the space domain through finite element (see also the references [16] and [17]). The problem of this direct integration methodology with step by step schemes is that, eventually, it is an approximation in the time domain by the time step parameter, thus, to obtain enough accurate solutions, with a sufficiently low damping rate imposed by the standard [18], it implies to define very small time sampling with numerical cost that it implies. To solve this problem several references appear like article Dugush and Eisenberger [19] which proposes a description of the modes of vibration by infinite polynomial series. Thus, the space is exactly solved by dynamic stiffness method and the analytical solution in the time domain thus corresponding to an infinite series. Another method is the so-called semi-analytical proposed by A. M. Castro et al [1], [20], [21] and [22], from the University of Granada. This methodology is based on the spatial resolution by finite element discretization and analytical resolution in the domain of time, thus all the approximation errors inherent in the time methods step by step will disappear. This semi-analytical method, although still in the first steps on its development, has been recognized by the scientific community and has been successfully applied in the design with Santa Ana bridge, arch bridge Córdoba-Málaga line [23].

In this research, the new Semi-Analytic methodology is taken as the basis of a new proposed one, dq/dv method. This proposed methodology determines the response of structures subjected to moving loads through the sensitivity of the maximum response to the train velocity. This sensitivity can be determined analytically thanks to the great advantage of the Semi-Analytic method, it is analytic in the time domain.

\subsubsection{Hilbert's transformation and its applications to engineering}

The Hilbert transform is published in 1905 by David Hilbert as an operator to solve a special case of the Riemann-Hilbert problem for holomorphic functions. Since then, is has been enormously developed and nowadays it has a wide range of applicability in signal conditioning. It was in 1946, [24], the first time it was proposed to consider a random signal $\mathrm{f}(\mathrm{t})$ as a product of two other independent function: $f(t)=A \cdot \cos (\varphi)$. Since then, researches and engineers have developed the concepts of envelope and instantaneous phase as well as the analytic signal and its treatment in the complex domain. Here we must mention an 
important result called the Bedrosian identity or product theorem, [25], which formulates the Hilbert transformation for the product of an oscillating and non-oscillating signals. An exhaustive review of all the theoretical concepts of Hilbert's transformation and its application to vibration analysis can was developed by M. Feldman [26] (2011). In civil engineering, there are plenty of applications of Hilbert transformation. In dynamic analysis, Hilbert transformation is a suitable tool to work with non-linear systems. We can mention the study presented by M. Simon and G. R. Tomlinson (1984)[27] in which it was set the basics of extended modal analysis to the domain of non-linear systems through Hilbert transformation. In the same line, there are other works as the one published by G. R. Tomlinson (1987) [28],in which it is described some developments in the use and application of Hilbert transform to identify non-linearity associated with simulated and experimental frequency response functions, or the works published by M. Feldman (1994) [29], once again, relative to techniques for non-linear system to identify instantaneous modal parameters as natural frequencies, damping characteristics and their dependencies on a vibration amplitude and frequency. In 1998 an outstanding work by Huand gave a new push to the modern research in the field of the HT, [30]. His original technique, known as the Empirical Mode Decomposition (EMD), adaptively decomposes a signal into the simplest intrinsic oscillatory modes (components) at the first stage. Then, a second stage, each decomposed component forms a corresponding instantaneous amplitude and frequency. Signal decomposition is a powerful approach; it has become extremely popular in various areas including nonlinear and non-stationary mechanics and acoustics. Finally, we can remark the work of Z. K, Penga en al. (2005) [31] that expose a new methodology based on a improved Hilbert-Huang transform to analyze non-stationary signals. This new tendency in signal analysis based on improved HilbertHuang has favored the appearance of many investigations, for instance the work of Olson (2007) [32] in which it is studied the dynamic bridge substructure condition assessment with Hilbert-Huang transform, applications to fail detection as works of Z. K. Penga et al. [33] in which HHT is applied to fault diagnosis for rolling bearing, earthquake ground motions [34], time-varying system identification [35], etc...

However, despite existing a huge number of publications relative to Hilbert transformation of signals, most of them are concerned on random signals for which it is not possible to obtain its exact analytic expression, thus, it is necessary to employ numerical approximations. In this present research, it is presented a methodology of dynamic analysis of structures subjected to moving load through the analytic expression of Hilbert transform of the signals of dynamic responses of the structure. The basis of this new methodology is the Semi-Analytic method which is exact in the time domain, this characteristic let the analytic integration of the Hilbert transform. 


\section{CHAPTER 2}

\section{Method 1: dq/dv. Derivate of the maximum acceleration respect to the train velocity}

\subsection{Introduction}

The finality of dynamic analysis of high speed rail bridges is to obtain the envelope of the maximum responses of a structure under the passage of moving loads. Moreover, the response can be defined by a two dependent variable function: velocity of the train and time. With this, the geometric sense of this analysis lays on the determination of the maximum envelope curve of the velocity uni-parametric family of curves time-response. This scheme can be understood more clearly on figure 2.1:

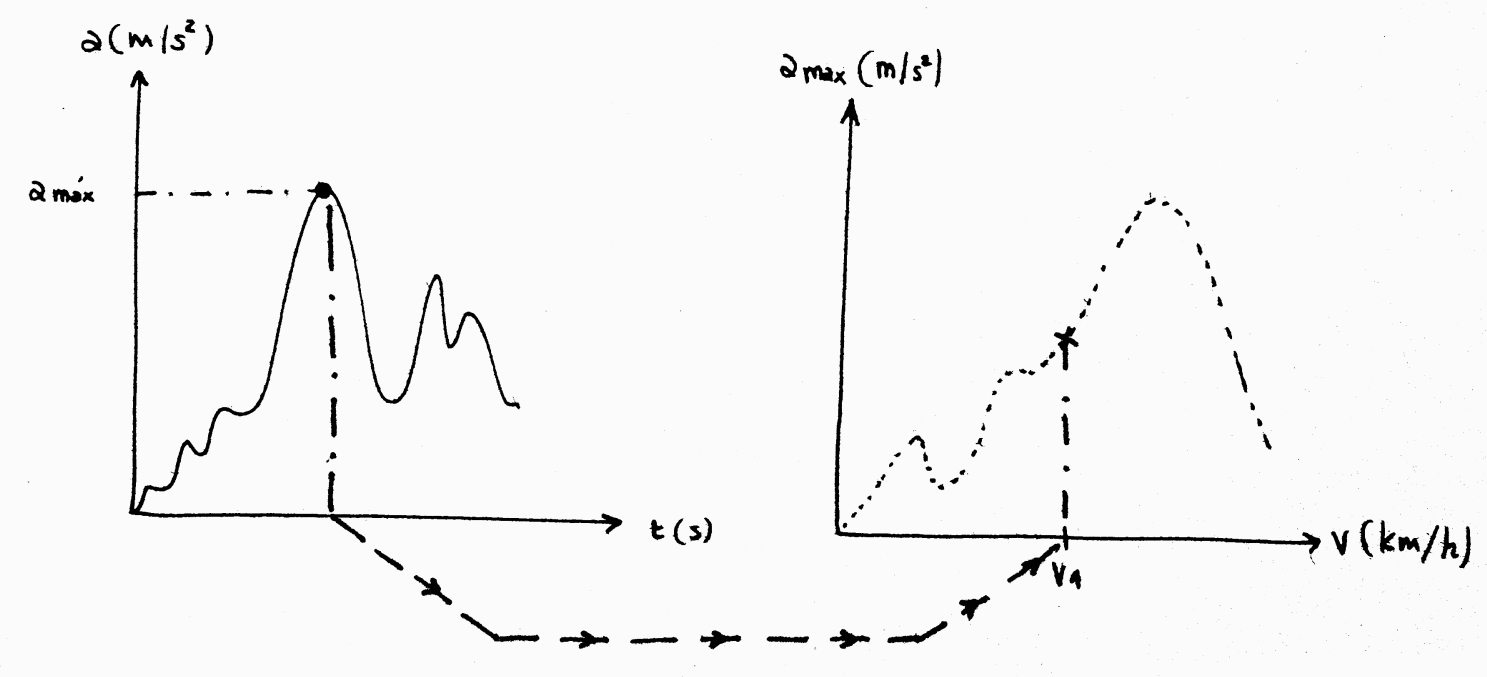

Figure 2.1: Obtaining of one point of maximum envelope curve in dynamic analysis.

In this figure, for several velocities, through step by step methods or Semi-Analytic solution, it can be determined the response of the structure (displacemnt and derivates) for each one of these velocity values. All this curves define a surface of response through a uniparametric family or curves with velocity as a single parameter and the dependent variables time and response. Because of this, the mathematical scheme is just the definition of the maximum envelope curve to this tridimensional surface, as can be seen in figure 2.2: 

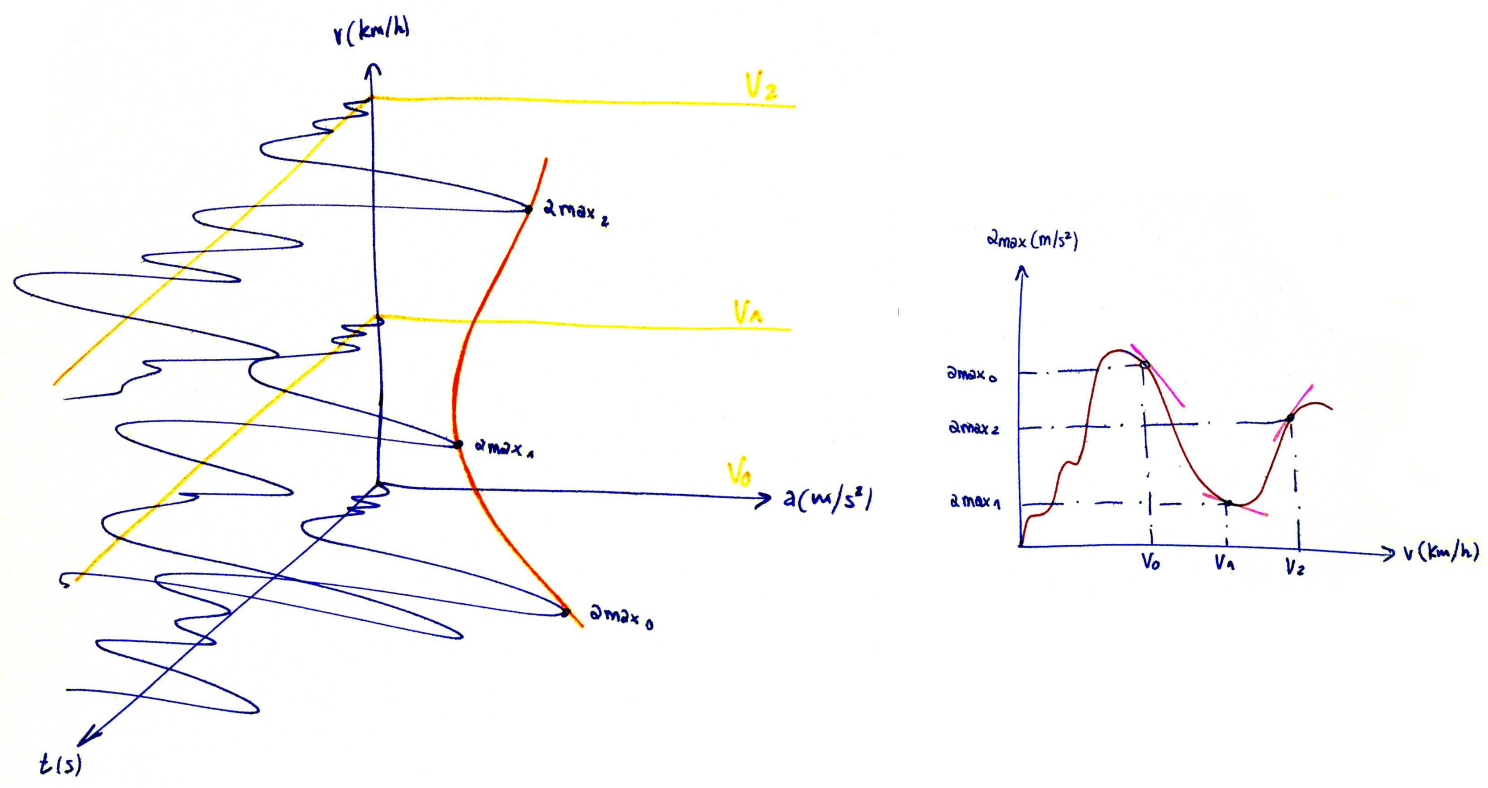

Figure 2.2: Obtaining of maximum envelope curve in dynamic analysis.

Due to the nature of the problem, the process can be divided in two steps: time series determination and maximum envelope definition. The first step obtains the response of the structure to a fixed velocity of the loads, and the second one collect the maximum values of these series and construct the curve maximum response vs velocity.

In this scheme, the aim of this project is to propose to new metamodels in order to carry out the direct integration of the time series which could improve the process in terms of time of calculus. The first one, is called Dq/dv method. This a method, based on SemiAnalytic solution, takes advantage of the condition of C3 class of the analytical signals obtained with Semi-Analytic solution, condition that permits the definition of the derivative of the response to the train velocity. Because of this, it is possible to set higher velocity steps and, posteriorly, fit a cubic spline between each two consecutive points in the envelope curve. With this, the number of direct calculations get decreased drastically and the obtained solution is accurate enough in pre-design phases.

\subsection{Theoretical development}

Semi-Analytic solution to the problem of moving loads on a structure can be written in a compact form:

$$
u(t, v)=C \cdot(q h(t, v)+q p(t, v))
$$

Being $\mathrm{u}$ the vertical displacement of the post-process point, $\mathrm{C}$ a modal matrix that arises from modal analysis, qh and qp, homogeneous and particular solutions to the differential solution after modal decomposition, and $t$ the time of the load over the element. To the proposal of a widest sampling steps, it is proposed a cubic interpolation spline between points 
of the envelope curve by the maximum value of the variable and it's first derivative. Because of this, the problem revolves around the determination of this mentioned derivative:

2.2.1 $d u / d v:$

$$
\frac{\partial u}{\partial v}=C \cdot\left(\frac{\partial q h}{\partial v}+\frac{\partial q p}{\partial v}\right)+\frac{L}{v^{2}} \cdot \frac{\partial u}{\partial t}
$$

This last term, taking into account that the time would be actually the time that goes after the time that the load requires to reach the initial point of the element:

$\left(t-\frac{L}{v}\right)$.

Analyzing separately both parts of the solution:

- Particular solution:

It can be written in a compact way:

$$
\begin{gathered}
q p=\left(\varphi_{v}\right)^{T} \cdot A \cdot \varphi_{t} \\
\varphi_{v}=\left(\begin{array}{c}
v^{3} \\
v^{2} \\
v \\
1
\end{array}\right) ; \varphi_{t}=\left(\begin{array}{c}
t^{3} \\
t^{2} \\
t \\
1
\end{array}\right) ; A=\left(\begin{array}{cccc}
\alpha_{31} & \alpha_{21} & \alpha_{11} & \alpha_{01} \\
0 & \alpha_{22} & \alpha_{12} & \alpha_{02} \\
0 & 0 & \alpha_{13} & \alpha_{03} \\
0 & 0 & 0 & \alpha_{04}
\end{array}\right)
\end{gathered}
$$

In this way, the searched derivative can be trivially obtained as:

$$
\begin{aligned}
& \frac{\partial q p}{\partial v}=\frac{d\left(\varphi_{v}\right)^{T}}{v} \cdot A \cdot \varphi_{t}=\left(\begin{array}{llll}
3 v^{2} & 2 v & 1 & 0
\end{array}\right) \cdot\left(\begin{array}{cccc}
\alpha_{31} & \alpha_{21} & \alpha_{11} & \alpha_{01} \\
0 & \alpha_{22} & \alpha_{12} & \alpha_{02} \\
0 & 0 & \alpha_{13} & \alpha_{03} \\
0 & 0 & 0 & \alpha_{04}
\end{array}\right) \cdot\left(\begin{array}{c}
t^{3} \\
t^{2} \\
t \\
1
\end{array}\right)= \\
& =\left(\begin{array}{llll}
A_{3} & A_{2} & A_{1} & A_{0}
\end{array}\right) \cdot\left(\begin{array}{c}
t^{3} \\
t^{2} \\
t \\
1
\end{array}\right) \\
& A 3=3 v^{2} \alpha_{31} \\
& A 2=3 v^{2} \alpha_{21}+2 v \alpha_{22} \\
& A 1=3 v^{2} \alpha_{11}+2 v \alpha_{12}+\alpha_{13} \\
& A 0=3 v^{2} \alpha_{01}+2 v \alpha_{02}+\alpha_{03}
\end{aligned}
$$


Now it is interesting to define this derivative in a similar scheme as the expression of q:

$$
\begin{aligned}
\frac{\partial q p}{\partial v} & =\beta_{0}+\beta_{1} \cdot(v t)+\beta_{2} \cdot(v t)^{2}+\beta_{3} \cdot(v t)^{3} \\
\beta_{0} & =A 0=3 v^{2} \alpha_{01}+2 v \alpha_{02}+\alpha_{03} \\
\beta_{1} & =A 1 / v=3 v \alpha_{11}+2 \alpha_{12}+\frac{\alpha_{13}}{v} \\
\beta_{2} & =A 2 / v^{2}=3 \alpha_{21}+\frac{2 \alpha_{22}}{v} \\
\beta_{3} & =A 3 / v^{3}=\frac{3 \alpha_{31}}{v}
\end{aligned}
$$

- Homogeneous solution:

$$
\begin{aligned}
& q h=e^{-\xi w t} \cdot\left(C 1(v, w, \xi) \cdot \cos \left(w_{D} t\right)+C 2(v, w, \xi) \cdot \operatorname{sen}\left(w_{D} t\right)\right) \\
& C 1=q(0)-\left(\begin{array}{llll}
v^{3} & v^{2} & v & 1
\end{array}\right) \cdot\left(\begin{array}{c}
\alpha_{01} \\
\alpha_{02} \\
\alpha_{03} \\
\alpha_{04}
\end{array}\right) \\
& C 2=\frac{\dot{q}(0)+\xi w C 1}{w_{D}}-\frac{1}{w_{D}} \cdot\left(\begin{array}{llll}
v^{3} & v^{2} & v & 0
\end{array}\right) \cdot\left(\begin{array}{c}
\alpha_{11} \\
\alpha_{12} \\
\alpha_{13} \\
0
\end{array}\right)
\end{aligned}
$$

As a result, its derivative:

$$
\begin{aligned}
\frac{\partial q h}{\partial v} & =e^{-\xi w t} \cdot\left(C^{\prime} 1 \cdot \cos \left(w_{D} t\right)+C^{\prime} \cdot \operatorname{sen}\left(w_{D} t\right)\right) \\
C^{\prime} 1 & =\frac{\partial q(0)}{\partial v}-\left(\begin{array}{llll}
3 v^{2} & 2 v & 1 & 0
\end{array}\right) \cdot\left(\begin{array}{c}
\alpha_{01} \\
\alpha_{02} \\
\alpha_{03} \\
\alpha_{04}
\end{array}\right)= \\
& =\frac{\partial q(0)}{\partial v}-\beta_{0} \\
C^{\prime} 2 & =\frac{\partial \dot{q}(0)}{\partial v}+\frac{\xi w}{w_{D}} \cdot C^{\prime} 1-\frac{1}{w_{D}} \cdot\left(\begin{array}{llll}
3 v^{2} & 2 v & 1 & 0
\end{array}\right) \cdot\left(\begin{array}{c}
\alpha_{11} \\
\alpha_{12} \\
\alpha_{13} \\
0
\end{array}\right)= \\
& =\frac{\frac{\partial \dot{q}(0)}{\partial v}+\xi w C 1-v \cdot \beta_{1}}{w_{D}}
\end{aligned}
$$

By this way, it can be set a structure to the sensitivity to velocity similar to the original form of displacement, so it can be understood as the resolution of the differential equation of the motion with a new load term, with a particular and homogeneous solutions as well as boundary conditions and inter-elemental continuity conditions.

2.2.2 dï/dv:

From the latter section it is obtained: 


$$
\begin{aligned}
\frac{\partial u}{\partial v} & =C \cdot\left(\frac{\partial q p}{\partial v}+\frac{\partial q h}{\partial v}\right)+\frac{L}{v^{2}} \cdot \frac{\partial u}{\partial t} \\
\frac{\partial q p}{\partial v} & =\beta_{0}+\beta_{1} \cdot(v t)+\beta_{2} \cdot(v t)^{2}+\beta_{3} \cdot(v t)^{3} \\
\frac{\partial q h}{\partial v} & =e^{-\xi w t} \cdot\left(C 11 \cdot \cos \left(w_{D} t\right)+C^{2} 2 \cdot \operatorname{sen}\left(w_{D} t\right)\right)
\end{aligned}
$$

Expressions whose second derivative respect to time is trivial:

$$
\begin{gathered}
\frac{\partial^{2}\left(\frac{\partial q p}{\partial v}\right)}{\partial t^{2}}=2 \cdot \beta_{2}+6 \cdot v t \cdot \beta_{3} \\
\frac{\partial^{2}\left(\frac{\partial q h}{\partial v}\right)}{\partial t^{2}}=e^{-\xi w t} \cdot\left(D 1 \cdot \cos \left(w_{D} t\right)+D 2 \cdot \operatorname{sen}\left(w_{D} t\right)\right) \\
D 1=C^{\prime} 1 \cdot\left((\xi w)^{2}-\left(w_{D}\right)^{2}\right)-C^{2} \cdot\left(2 \xi w w_{D}\right) \\
D 2=C^{\prime} 2 \cdot\left((\xi w)^{2}-\left(w_{D}\right)^{2}\right)+C^{\prime} 1 \cdot\left(2 \xi w w_{D}\right) \\
\frac{\partial^{2}\left(\frac{\partial q}{\partial v}\right)}{\partial t^{2}}=2 \cdot \beta_{2}+6 \cdot v t \cdot \beta_{3}+e^{-\xi w t} \cdot\left(D 1 \cdot \cos \left(w_{D} t\right)+D 2 \cdot \operatorname{sen}\left(w_{D} t\right)\right)+ \\
+\frac{L}{v^{2}} \cdot \frac{\partial^{3} q}{\partial t^{3}}
\end{gathered}
$$

\subsubsection{Applicability to maximum envelope determination}

In order to obtain the derivative of the envelope function from the derivative of the time series some mathematical results are needed.

To obtain more general results, foreseeing possible extensions of this methodology in the future, the basis of the theory in which all the results are developed is the field of vectorial functions: Let:

$$
\begin{aligned}
& \bar{f}: R^{n} \longmapsto R^{m} \\
& \bar{f}=\left(f_{1}, \ldots ., f_{i}, \ldots ., f_{m}\right) ; f i=f i\left(x_{1}, \ldots ., x_{i}, \ldots ., x_{n}\right)
\end{aligned}
$$

Thus, it is defined the diferential of $\bar{f}$ as follows:

$$
d \bar{f}(x)=\left\{\begin{array}{c}
d f_{1} \\
\ldots \\
d f_{i} \\
\ldots \\
d f_{n}
\end{array}\right\}=\left\{\begin{array}{ccc}
\frac{\partial f_{1}}{\partial x_{1}} & \ldots & \frac{\partial f_{1}}{\partial x_{n}} \\
\ldots . & & \ldots . \\
\frac{\partial f_{m}}{\partial x_{1}} & \ldots & \frac{\partial f_{m}}{\partial x_{n}}
\end{array}\right\}\left\{\begin{array}{c}
d x_{1} \\
d x_{n}
\end{array}\right\}=\left[D \bar{f}\left(x_{1}, \ldots, x_{n}\right)\right]\{d x\}
$$

Invertible vector function:

A biyective function $\bar{f}: \mathrm{I} \subset R^{n} \longmapsto R^{m}$ admits one single inverse function $\bar{g}=\bar{f}(\mathrm{I}) \subset R^{m} \longmapsto \mathrm{I}$ which:

$$
\bar{g}(\bar{f}(a))=a, \forall a \in I
$$

Local invertibility theorem

Definition:

It is said that $\bar{f}: \mathrm{I} \subset R^{n} \longmapsto R^{m}$ is locally invertible in $\mathrm{a} \in \mathrm{I}$ if there is a set $\mathrm{V}(\mathrm{a})$ in which $\mathrm{f} \|_{V(a)}: \mathrm{V}(\mathrm{a}) \subset R^{n} \longmapsto R^{m}$ is invertible. 
Invertibility theorem:

Let I an open set of $R^{n}$, one function $\bar{f}: \mathrm{I} \subset R^{n} \longmapsto R^{m}$ of components $\left(f_{1}, f_{2} \ldots, f_{n}\right)$ that are continuous and with continuous partial derivatives in $a$ is locally invertible if:

$$
J(a)=\operatorname{det}[D \bar{f}(a)] \neq 0
$$

If $\mathrm{a} \in \mathrm{I}$, the local inverse function of $\bar{f}$ which corresponds to the point $\mathrm{a}$ is defined in a set $\mathrm{V}(\bar{f}(\mathrm{a})) \subset R^{n}$ around the point $\bar{f}(\mathrm{a})$ and it is continuous in $\bar{f}(\mathrm{a})$, with continuous partial derivatives in $\bar{f}(\mathrm{a})$.

\section{Implicit Function Theorem}

Definition:

It is defined a vectorial function $\mathrm{g}: \Omega \longmapsto R^{m}$, with $\Omega \subset R^{k} x R^{m}$ and $(\mathrm{a}, \mathrm{b}) \in \Omega$ as a point in which $g(a, b)=0$, it can be said that the equation $g(x, y)=0$ defines, in a set of $(a, b)$, the variable $y \in R^{m}$ a implicit function of the variable $\mathrm{x} \in R^{k}$ if there is an open set $\mathrm{A}$ around $\mathrm{a}$, and an open set $\mathrm{B}$ around $b$, as $A x B \subset \Omega$ and for each $x \in A$ there is an only $y=f(x) \in B$ which verifies $g(x, f(x))=0$. It is then said that $\mathrm{f}: \mathrm{A} \longmapsto \mathrm{B}$ is the implicit function of the equation $\mathrm{g}(\mathrm{x}, \mathrm{y})=0$.

Theorem

Let $\mathrm{g}: \Omega \longmapsto R^{m}$ a function of derivability $C^{p}(\Omega)\left(C^{p} \geq 1\right)$ defined in an open set $\Omega \subset R^{k} \times R^{m}$ and $(a, b) \in \Omega$ is a point that satisfies $g(a, b)=0$. If the determinant of the jacobian form of the components of $g$ respect to variables $\left(y_{1}, y_{2}, \ldots, y_{m}\right)$ is not null in $(a, b)$ :

$$
\frac{D\left(g_{1}, g_{2}, \ldots, g_{m}\right)}{D\left(y_{1}, y_{2}, \ldots, y_{m}\right)}(a, b) \neq 0
$$

Then $\mathrm{g}(\mathrm{x}, \mathrm{y})=0$ defines, in a set of $(\mathrm{a}, \mathrm{b})$, the variable $\mathrm{y}=\left(y_{1}, y_{2}, \ldots, y_{m}\right)$ as implicit function of $\mathrm{x}=\left(x_{1}, x_{2}, \ldots, x_{k}\right)$, i. e., there is $\mathrm{AxB} \subset \Omega$, open set of $(\mathrm{a}, \mathrm{b})$ so that for each $\mathrm{x} \in \mathrm{A}$ there is a single $\mathrm{y}=\mathrm{f}(\mathrm{x}) \in \mathrm{B}$ that verifies $\mathrm{g}(\mathrm{x}, \mathrm{y})=0$. Besides, the implicit function $\mathrm{f}: \mathrm{A} \longmapsto \mathrm{B}$ has a derivability of $C^{p}(\mathrm{~A})$.

\section{Demostration}

Let $\mathrm{G}: \Omega \longmapsto R^{k} \mathrm{x} R^{m}$, defined by $\mathrm{G}(\mathrm{x}, \mathrm{y})=(\mathrm{x}, \mathrm{g}(\mathrm{x}, \mathrm{y}))$, is $C^{p}(\Omega)$ and verifies:

$$
\operatorname{det} \hat{G}(a, b)=\frac{D\left(g_{1}, g_{2}, \ldots, g_{m}\right)}{D\left(y_{1}, y_{2}, \ldots, y_{m}\right)}(a, b) \neq 0
$$

If the inverse theorem is applied to $\mathrm{G}$, two open sets $\mathrm{U}, \mathrm{V} \subset R^{n}$ can be defined with $(\mathrm{a}, \mathrm{b}) \subset$ $\mathrm{U}$ and $\mathrm{G}(\mathrm{a}, \mathrm{b})=(\mathrm{a}, 0) \in \mathrm{V}$, such as $\mathrm{G} \|_{U}: \mathrm{U} \longmapsto \mathrm{V}$ is a biyection with inverse $\mathrm{F}: \mathrm{V} \longmapsto \mathrm{U}$ and its derivability is $C^{p}$. Let a set $\mathrm{U}=A_{0} \times B_{0}$ with $A_{0}$ an open set around a and $B_{0}$ another open set of $\mathrm{b}$. It is defined $\forall(\mathrm{u}, \mathrm{v}) \in \mathrm{V}$, the inverse function in the form $\mathrm{F}(\mathrm{u}, \mathrm{v})=\left(F_{1}(\mathrm{u}, \mathrm{v}), F_{2}(\mathrm{u}, \mathrm{v})\right)$.

In the same way as $\mathrm{G}$ fixes its first invariables $\mathrm{F} 1(\mathrm{u}, \mathrm{v})=\mathrm{u} \forall(\mathrm{u}, \mathrm{v}) \in \mathrm{V}$. It is verified: 


$$
\begin{aligned}
(u, v) & =G(F(u, v))=G\left(u, F_{2}(u, v)\right)=\left(u, g\left(u, F_{2}(u, v)\right)\right. \\
v & =g\left(u, F_{2}(u, v)\right)
\end{aligned}
$$

Then, the function $\mathrm{f}: \mathrm{A} \longmapsto R^{m}$, defined in $\mathrm{A}:\left\{\mathrm{x} \in R^{k}:(\mathrm{x}, 0) \in \mathrm{V}\right\}$, by $\mathrm{f}(\mathrm{x})=F_{2}(\mathrm{x}, 0)$ satisfies $\mathrm{g}(\mathrm{x}, \mathrm{f}(\mathrm{x}))=0 \forall \mathrm{x} \in \mathrm{A}$, being $\mathrm{A}$ an open set around a. Furthermore, $\mathrm{A} \subset A_{0}$ and $\mathrm{f}(\mathrm{A}) \subset B_{0}$, as:

$$
x \in A \Rightarrow(x, 0) \in V \Rightarrow F(x, 0)=(x, f(x)) \in U=A_{0} x B_{0} .
$$

With $\mathrm{B}=B_{0}$ it is accomplished $\mathrm{f}(\mathrm{A}) \subset \mathrm{B}$, and $\mathrm{g}(\mathrm{x}, \mathrm{f}(\mathrm{x}))=0 \forall \mathrm{x} \in \mathrm{A}$. If $\mathrm{y} \in \mathrm{B}$ and $\mathrm{g}(\mathrm{x}, \mathrm{y})=0$ $\Rightarrow \mathrm{G}(\mathrm{x}, \mathrm{y})=(\mathrm{x}, \mathrm{g}(\mathrm{x}, \mathrm{y}))=(\mathrm{x}, 0) ; \mathrm{G}(\mathrm{x}, \mathrm{f}(\mathrm{x}))=\left(\mathrm{x}, \mathrm{g}(\mathrm{x}, \mathrm{f}(\mathrm{x}))=(\mathrm{x}, 0)\right.$. Taking into consideration that $\mathrm{G} \|_{U}$ is inyective, and that the points $(x, y),(x, f(x))$ are into $A x B \subset A_{0} x B_{0}=0$ it can be set $y=f(x)$, as the desired implicity function. Finally its derivability is $C^{p}(\mathrm{~A})$ because $\mathrm{F}^{\prime} \mathrm{s}$ derivability is $C^{p}(\mathrm{~V})$. Calculus of partial derivatives of implicit functions

In the same conditions as the latter theorem, when g's derivability is $C^{p}$, altough the determination of the explicit equation of the implicit function $\mathrm{f}$ could not be determined and then its derivative, it is possible to calculate successive partial derivatives (until p order) locally in the point $(a, b)$. With that, in the case of $k=1 \mathrm{y} m=2$, the set in which it is located this problem, let a function $g$ with successive partial derivatives in a region $U \subset R^{2}$ and let $\left(x_{0}, y_{0}\right)$ an interior point to $\mathrm{U}$ so that $\mathrm{f}\left(x_{0}, y_{0}\right)=0$ and the derivative respect to the parameter is not null $f_{y}\left(x_{0}, y_{0}\right) \neq 0$. Then, by the theorem of the implicit function, there is an interval I, centered on point $x_{0}$, and one single $\mathrm{f}: \mathrm{x} \in \mathrm{T} \subset \mathrm{R} \longmapsto \mathrm{y}(\mathrm{x}) \in \mathrm{R}$, derivable and with continuous derivatives in I so that $\mathrm{y}\left(x_{0}\right)=x_{0}$, being the solution of the implicit equation $\mathrm{f}(\mathrm{x}, \mathrm{y})=0 \forall \mathrm{x} \in \mathrm{I}$, besides, the derivative of function $y$ is defined by :

$$
f_{x}(x, y(x))+\dot{y}(x) \cdot f_{y}(x, y(x))=0 \longmapsto y ́ y(x)=-\frac{f_{x}(x, y(x))}{f_{y}(x, y(x))} \forall x \in I
$$

For the general case of vectorial functions $\mathrm{f}: \mathrm{YxA} \longmapsto \mathrm{R}$, with $\mathrm{y} \in \mathrm{Y} \subset R^{n} \mathrm{y} \mathrm{x} \in \mathrm{A} \subset R^{m}$, under the conditions of the theorem of the implicit function for a set $\mathrm{I} \subset \mathrm{A}$, the latter derivative can be defined locally by:

$$
D_{x} y(x)=-\left[D_{y} f(y(x), x)\right]^{-1} D_{x} f(y(x), x) \forall x \in I
$$

\section{Envelope Theorem}

The Envelope Theorem is one of the main applications of the theorem of the implicit function which refers to how the optimal value of a function varies when the parameter of the function changes. This theorem can be used in problems with and without restrictions, however, relating to the aim of the present research, the definition will only refers to formulation and demonstration of its application in problems without restrictions. In the case of solutions with restrictions, the theorem is based on Lagrange multipliers used in the optimization of functions written in its implicit form. 


\section{Theorem}

Let $\mathrm{f}: \mathrm{f}: \mathrm{YxA} \longmapsto \mathrm{R}$, with $\mathrm{y} \in \mathrm{Y} \subset R^{n}$ and $\mathrm{x} \in \mathrm{A} \subset R^{m}$, with the conditions of the theorem of the implicit function for a set $I \subset A$. The problem can be written as $\max \{f(y, x)\}$ for the value $y^{*}\left(x^{*}\right)$. In these conditions:

$$
D_{x} f\left(y^{*}\left(x^{*}\right), x^{*}\right)=D_{x} f(y(x), x) \|_{y=y *(x), x=x *}
$$

In the particular case of $n=1$ y $m=1, g(x, y): R \longmapsto R$, being $f(x)=\max _{y}\{g(x, y)\}$ :

$$
\frac{d f(x)}{d x}=\frac{\partial g}{\partial x} \|_{(x, y(x))}
$$

\section{Demostration}

The result is demostrated just by applying the chain rule for derivatives:

$$
\begin{aligned}
D_{x} f\left(y^{*}\left(x^{*}\right), x^{*}\right) & =D_{x} f(y(x), x) \|_{y=y *(x), x=x *}+\overbrace{D_{y} f(y(x), x) \|_{y=y *(x), x=x *}}^{=0} \cdot D_{x} y *(x)= \\
& =D_{x} f(y(x), x) \|_{y=y *(x), x=x *}
\end{aligned}
$$

If more information is needed the following references can be consulted [36], [37], [38], [39], [40], [41], [42] and [43], as the reference documentation used in the elaboration of this section.

\section{Aplication to the Semi-Analytic solution}

More specifically, the problem analyzed in this research is focused on the determination of the derivative of the envelope curve of accelerations in order to define a posterior cubic interpolation of the response. Because of this, it is determined the analytic expression of the uni-parametric family of curves of acceleration, with the velocity as the parameter. Therefore, with a known velocity sampling,$v^{*}$, it can be determined the value of the maximum acceleration, $a_{\max }$, the time in which it occurs, $t_{\max }$, and the derivative $\frac{\partial a}{\partial v} \|_{\left(t=t_{\max }\right)\left(v=v^{*}\right)}$. Nevertheless, it is necessary to evaluate the applicability of the mentioned derivative as because of working with a envelope curve, and being evaluated in a time $t=t m a x$ correspondent to the resonant behavior for the certain step $\mathrm{v}=v_{i}$, it is not trivial to ensure that this derivative will not significantly vary with an increment in velocity because of a non negligible change in the time in which the resonance appears.

From a mathematical point of view, the Semi-Analytic methodology accomplishes all the requirements of the theorem. In general terms:

$$
a=f(v, t), f: A x B \longmapsto R, A \subset R, B \subset R
$$

Defining $a_{\max }(\mathrm{v})=\max t\{\mathrm{a}(\mathrm{v}, \mathrm{t})\}$, curve with derivability $\mathrm{C} 1$, the envelope theorem sais: 


$$
\frac{d a_{\max }}{d v}=\frac{\partial a}{\partial v} \|_{t=t_{m} a x}
$$

Hence, despite the maximum values take place at different times, locally, the differences in time between the maximum response with $\mathrm{v}$ and $\mathrm{v}+\Delta v$ are negligible. Thus, the local scheme is summarized in figures 2.3 and 2.4, where the time in which the maximum values occur is approximately the same:
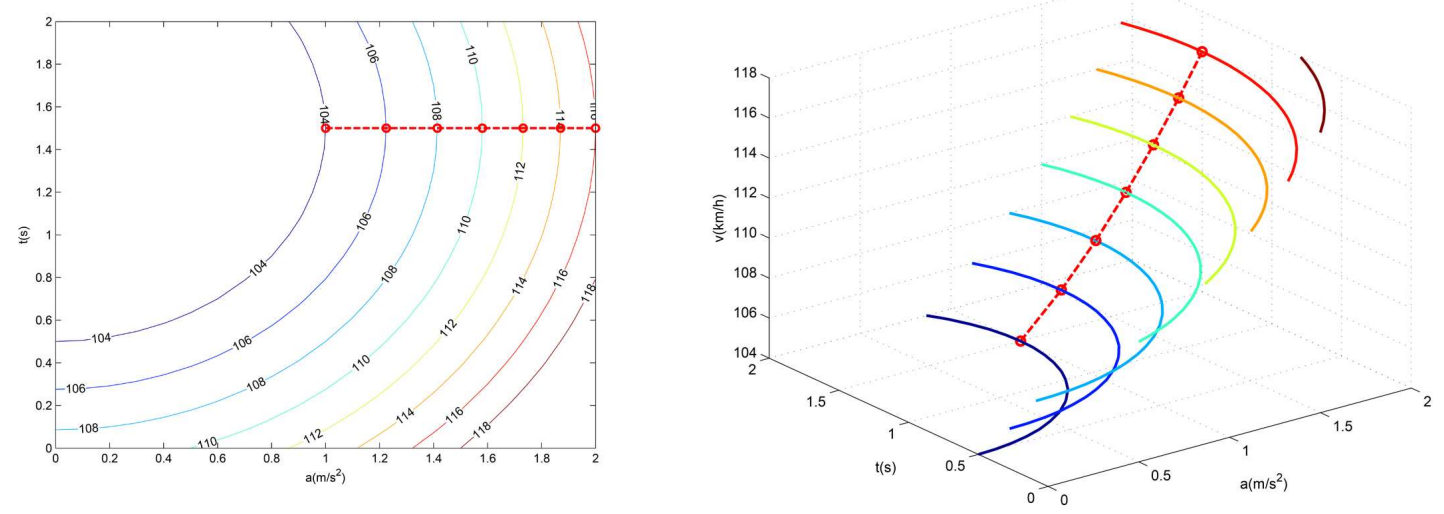

Figure 2.3: Scheme of local envelope curve of response in dynamic analysis. 


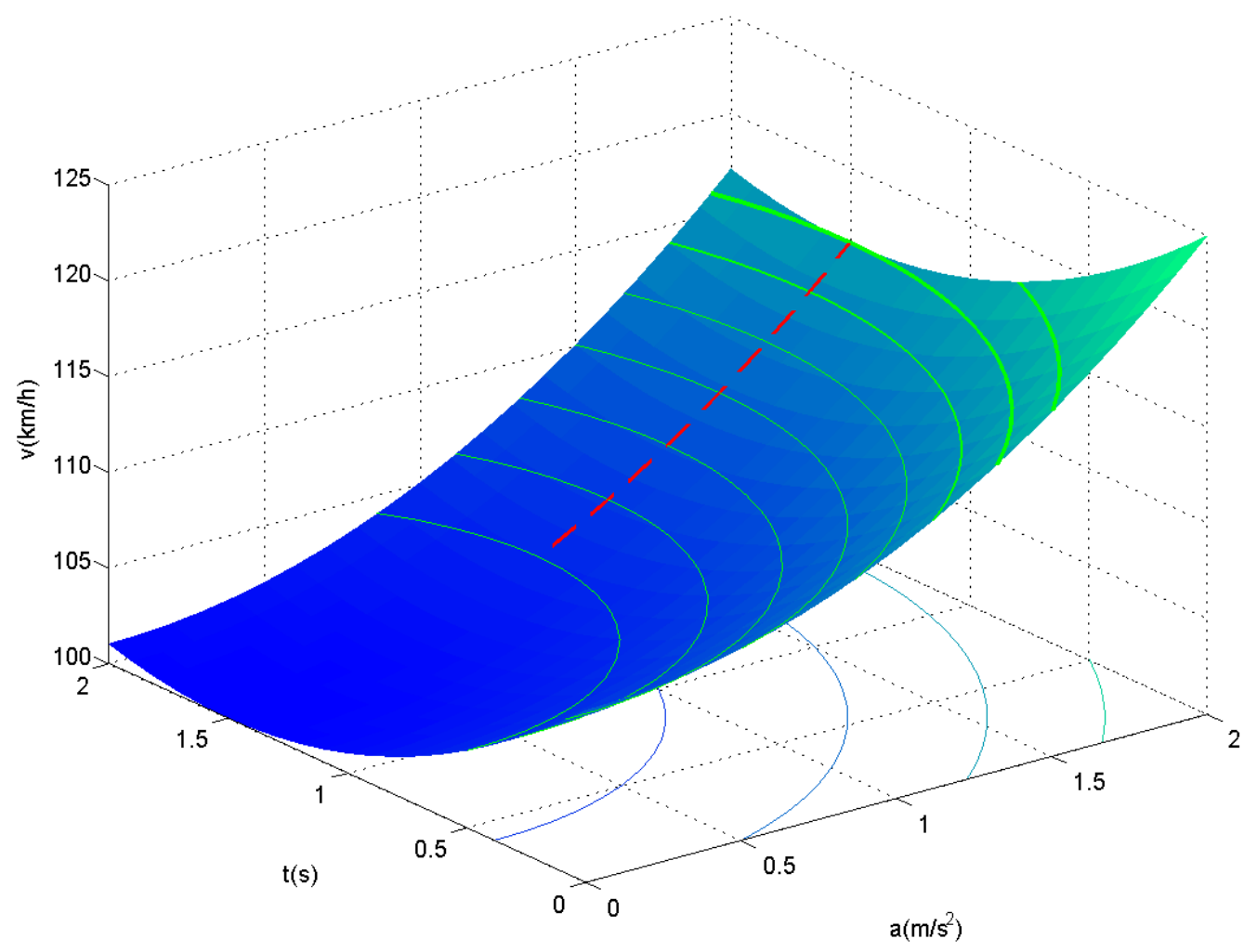

Figure 2.4: Local response surface of structure under moving loads.

Therefore, the procedure to follow, in this new proposed variant of the Semi-Analytic methodology, is to carry out a passage of the loads with higher velocity steps, collection of peaks and time in which they occur so that, after that, the expression $\frac{\partial a}{\partial v}$ can be evaluated in the obtained time. With this, a cubic interpolation of the envelope curve can be defined from the values of maximum accelerations and their sensibility to the variation of velocity.

\subsection{Test}

\subsubsection{Simply supported beam under a single moving load (IAPF)}

This example is analyzed in the Spanish normative IAPF (Standard of loads in railway bridges) and consist on a simply supported beam with a single $15 \mathrm{~m}$ span. The mass per unit length (the product of density and the cross-sectional area) is constant in the whole beam ant it has a value of $\rho A=15000 \mathrm{~kg} / \mathrm{m}$. The flexural stiffness $E I$ is $7694081 \mathrm{kNm}^{2}$. The damping coefficient ratio is $2 \%$. The reason of this choice is that we can compare the proposed method with an exact analytical solution just by solving the differential equation of motion. 


\section{EI, $\rho \mathrm{A}$}

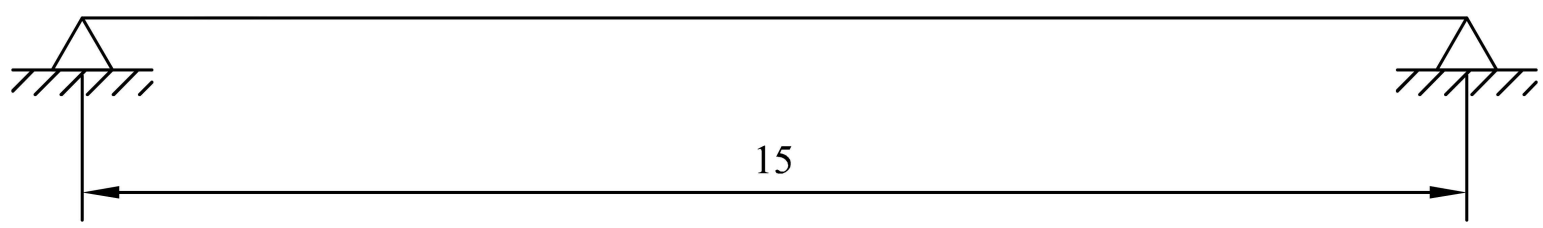

Figure 2.5: Simply supported beam

\section{Analytical Solution}

The differential equation of motion in a mass distributed Bernouilli-Euler beam:

$$
\rho(x) \cdot \frac{\partial^{2} u}{\partial t^{2}}+c(x) \cdot \frac{\partial u}{\partial t}+\frac{\partial^{2}}{\partial x^{2}} \cdot\left[E I(x) \cdot \frac{\partial^{2} u}{\partial x^{2}}\right]+p_{0} \cdot \delta(x-v t)=0
$$

Undamped free vibration

$$
\rho(x) \cdot \frac{\partial^{2} u}{\partial t^{2}}+\frac{\partial^{2}}{\partial x^{2}} \cdot\left[E I(x) \cdot \frac{\partial^{2} u}{\partial x^{2}}\right]=0
$$

Applying modal variable separation:

$$
\begin{gathered}
u=\Phi(x) \cdot Y(t) \\
E I \cdot \Phi(x)^{I V} \cdot Y+\rho \cdot \Phi \cdot \ddot{Y}=0 \\
\frac{E I \cdot \Phi^{I V}}{\Phi}=-\frac{\ddot{Y}}{Y} \cdot \rho=a^{4}
\end{gathered}
$$

Spacial Solution:

It can be supposed an exponential form for the term $\Phi(\mathrm{x})$ as $A \cdot e^{s x}$ :

$$
\begin{gathered}
s^{4}-a^{4}=0 \rightarrow s=\left\{\begin{array}{c} 
\pm a \\
\pm a i
\end{array}\right\} \\
\Phi(x)=C_{1} \cdot \operatorname{sen}(a \cdot x)+C_{2} \cdot \cos (a \cdot x)+C_{3} \cdot \operatorname{senh}(a \cdot x)+C_{4} \cdot \cosh (a \cdot x)
\end{gathered}
$$

Time Solution:

$$
\begin{gathered}
-\frac{\ddot{Y}}{Y} \cdot \frac{m}{E I}=a^{4} \\
Y=A \cdot \cos (w t)+B \cdot \operatorname{sen}(w t) \rightarrow w^{2} \cdot \frac{m}{E I}=a^{4} \\
a=\sqrt[4]{\frac{w^{2} \cdot m}{E I}}
\end{gathered}
$$

Boundary conditions:

$$
\Phi(0)=\Phi^{\prime \prime}(0)=\Phi(L)=\Phi "(L)=0
$$


Thereby:

$$
\begin{gathered}
C_{1}=C_{2}=C_{3}=0 \\
a \cdot L=n \cdot \pi \rightarrow a=\frac{n \pi}{L} \\
w=\sqrt{\frac{E I \cdot a^{4}}{m}}=\frac{n^{2} \cdot \pi^{2}}{L^{2}} \sqrt{\frac{E I}{m}}
\end{gathered}
$$

Free vibration

$$
\rho(x) \cdot \frac{\partial^{2} u}{\partial t^{2}}+c(x) \cdot \frac{\partial u}{\partial t}+\frac{\partial^{2}}{\partial x^{2}} \cdot\left[E I(x) \cdot \frac{\partial^{2} u}{\partial x^{2}}\right]=0
$$

Applying modal variable separation and pre-multiplying by $\Phi$, we can integrate and use the orthogonal properties of modes of vibration:

$$
\begin{gathered}
\int_{0}^{L} \Phi \cdot \frac{\partial^{2}}{\partial x^{2}} \cdot\left[E I(x) \cdot \frac{\partial^{2} u}{\partial x^{2}}\right] d x \cdot Y(t)+\int_{0}^{L} \Phi \cdot c \cdot \Phi d x \dot{Y}+\int_{0}^{L} \Phi \cdot m \cdot \Phi d x \ddot{Y}=0 \\
K n \cdot Y(t)+C n \cdot \dot{Y}+M n \cdot \ddot{Y}=0
\end{gathered}
$$

By admitting an equal damping ratio for all modes, the solution of the latter equation results:

$$
Y(t)=e^{-\xi w t} \cdot(A n \cdot \cos (w d t)+B n \cdot \operatorname{sen}(w d t))
$$

Forced vibration

$$
\begin{aligned}
& K n \cdot Y(t)+C n \cdot \dot{Y}+M n \cdot \ddot{Y}=p o \int_{0}^{L} \Phi(x) \delta(x-v t) d x \\
&=p o \Phi(v t)=p o \cdot \operatorname{sen}\left(\frac{n \pi v t}{L}\right) \\
& \ddot{Y}+2 \cdot \xi \cdot w n \cdot \dot{Y}+w n^{2} \cdot Y=\frac{2 \cdot p o}{m \cdot L} \cdot \operatorname{sen}\left(\frac{n \pi v t}{L}\right) ; \Omega=\frac{n \pi v t}{L}
\end{aligned}
$$

We can suppose a solution as follows:

$$
q=C 1 \cdot \cos (\Omega t)+C 2 \cdot \operatorname{sen}(\Omega t)
$$

And the constants can be determined just by substituting the latter expression into the differential equation of motion:

$$
\begin{gathered}
C 1=-2 \cdot \xi \cdot \frac{\frac{2 p L^{3}}{E I \cdot n^{4} \cdot \pi^{4}}}{\left(1-S n^{2}\right)^{2}+4 \cdot(\xi \cdot S n)^{2}} \\
C 2=\left(1-S n^{2}\right) \cdot \frac{\frac{2 p L^{3}}{E I \cdot n^{4} \cdot \pi^{4}}}{\left(1-S n^{2}\right)^{2}+4 \cdot(\xi \cdot S n)^{2}}
\end{gathered}
$$




$$
S n=\frac{\Omega}{w n}
$$

Therefore the complete solution results:

$$
\begin{aligned}
q n & =\frac{\frac{2 p L^{3}}{E ! \cdot n^{4} \cdot \pi^{4}}}{\left(1-S n^{2}\right)^{2}+4 \cdot(\xi \cdot S n)^{2}} \cdot\left\{\left(1-S n^{2}\right) \cdot \operatorname{sen}(\Omega t)-2 \xi \operatorname{Sn} \cdot \cos (\Omega t)+\right. \\
& \left.+e^{-\xi w n \cdot t} \cdot\left[2 \xi \cos (w d \cdot t)+\frac{S n}{\sqrt{1-\xi^{2}}} \cdot\left(2 \xi^{2}+S n^{2}-1\right) \cdot \operatorname{sen}(w d \cdot t)\right]\right\}
\end{aligned}
$$

\section{Solution by semianalytic method}

The beam is pre-analyzed by a finite element program, in this case SAP2000 v15. The structure is simulated by 24 linear elements and one single mode of vibration. The reason of this discretization is to get the same modal frequency as the analytical expression, $5 \mathrm{~Hz}$, taking into consideration that SAP2000 employs mass concentrated elements.

The load has a value of $9.8 \mathrm{kN}, 5 \mathrm{~m}$ from the origin of the structure and running at $200 \mathrm{~km} / \mathrm{h}$.

Displacements:

Analyzing the response of the mid-span point in terms of displacements, the proposed method can be compared, firstly, with the analytical solution and, finally, with the derivate obtained by Central Differences with the series of displacement obtained by the semianalytic solution at velocities $199.9 \mathrm{~km} / \mathrm{h}$ and $200.1 \mathrm{~km} / \mathrm{h}$.

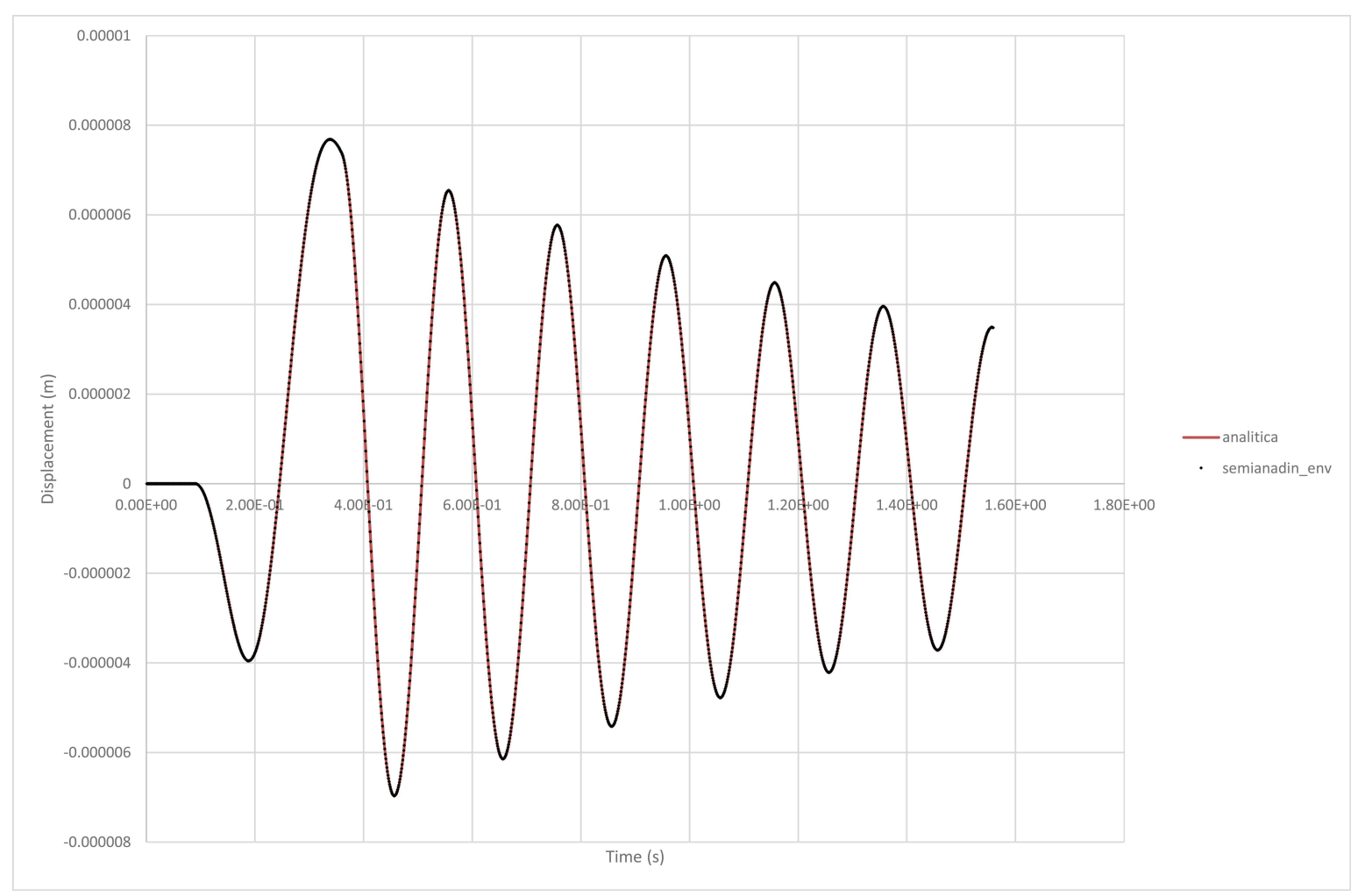

Figure 2.6: Semianadin vs Analytical 


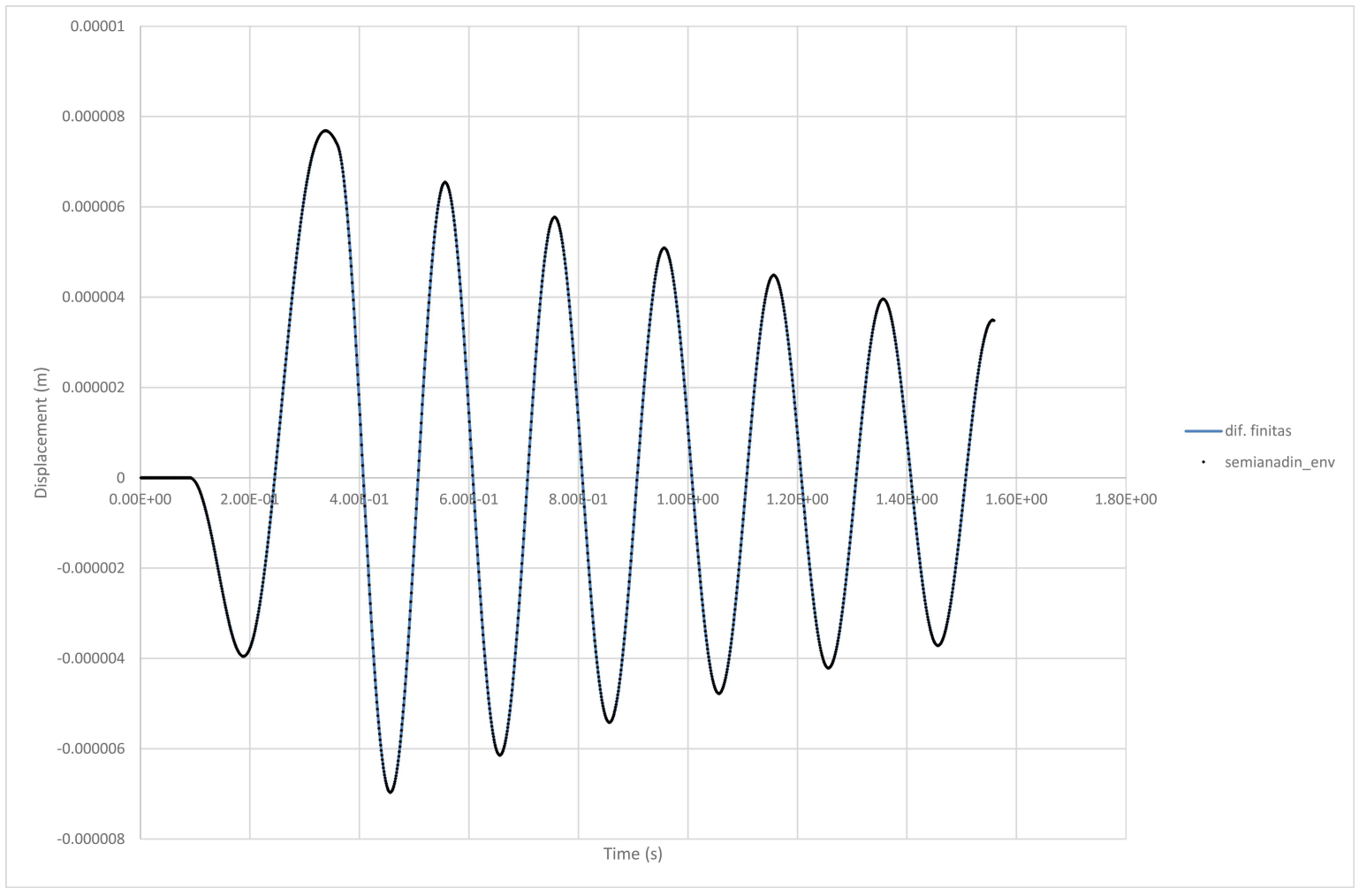

Figure 2.7: Semianadin vs Central difference derivatives

\section{Accelerations:}

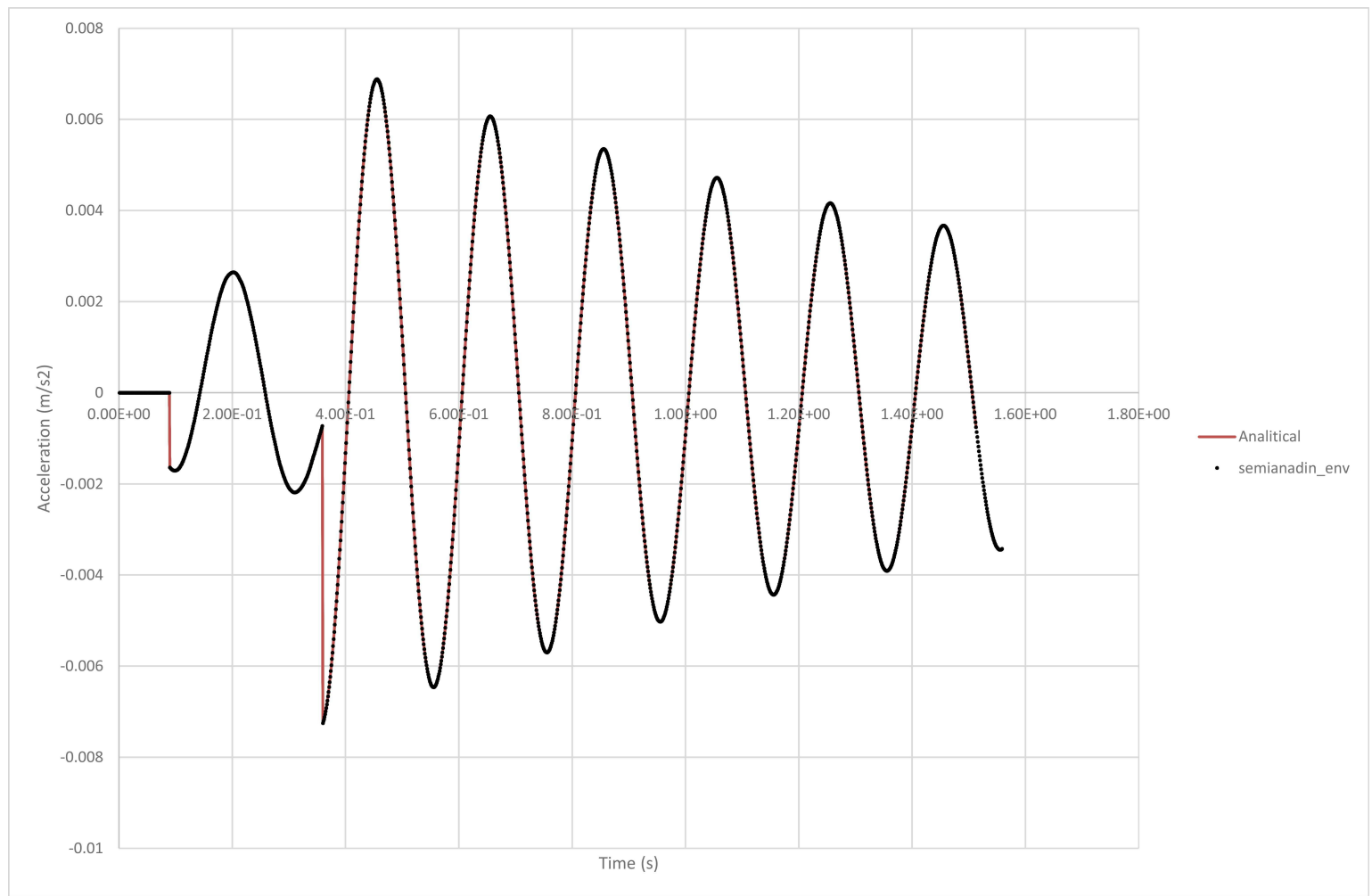

Figure 2.8: Semianadin vs Analytical 


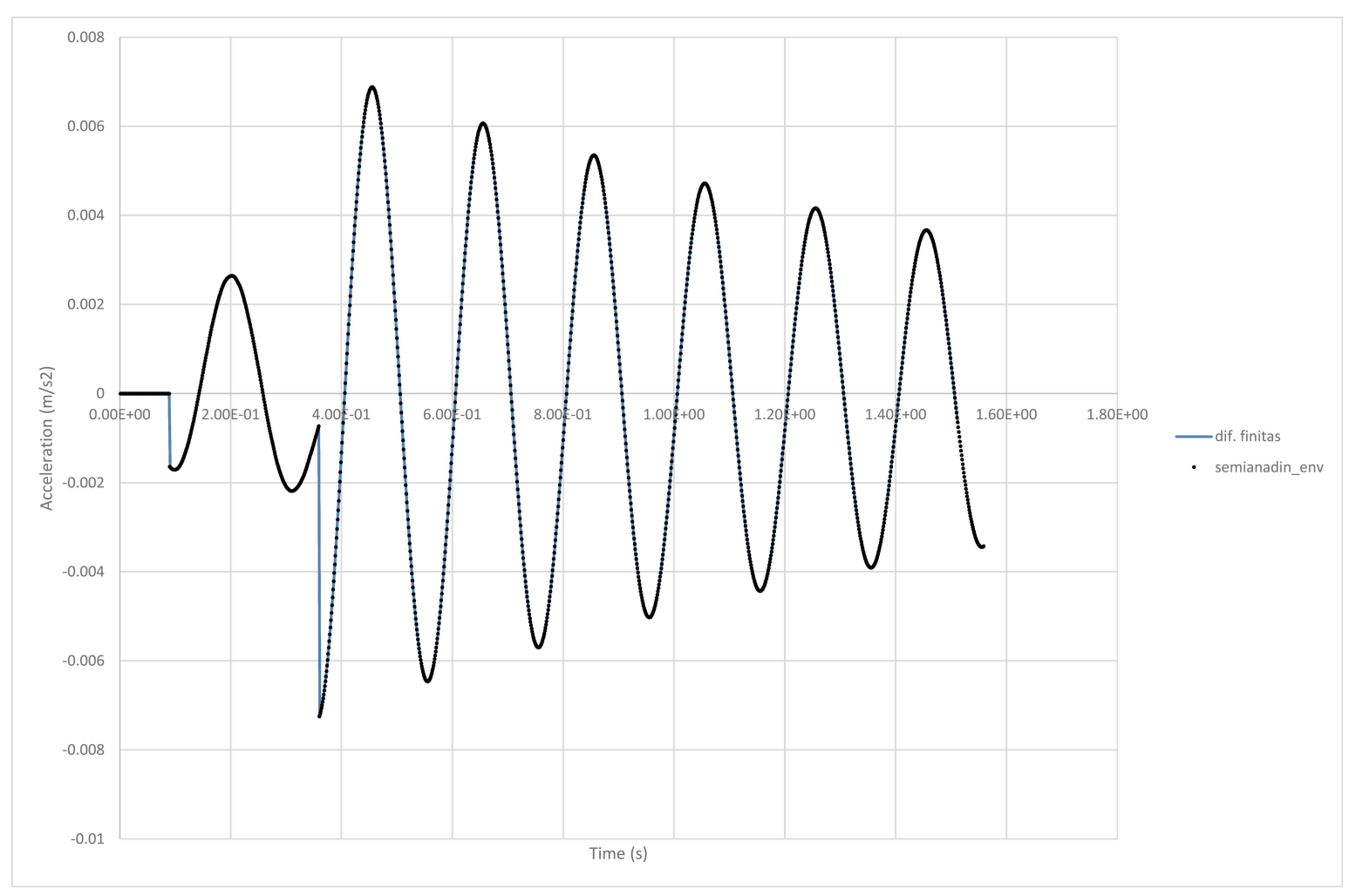

Figure 2.9: Semianadin vs Central difference derivatives

\subsubsection{A three-span continuous stepped beam under a single moving load. (Henchi).}

In this case, this study is applied to a well known structure in the bibliography [44]. It consists on a $20 \mathrm{~m}$ length three-span continuous stepped beam, the mass per unit length $\rho A$ is $1000 \mathrm{~kg} / \mathrm{m}$, and it is constant along all the structure. The flexural stiffness EI is 1.96 $\mathrm{GN} m^{2}$ for the lateral spans, and the central one has double flexural stiffness. The damping coefficient $\xi$ is set to $2 \%$.

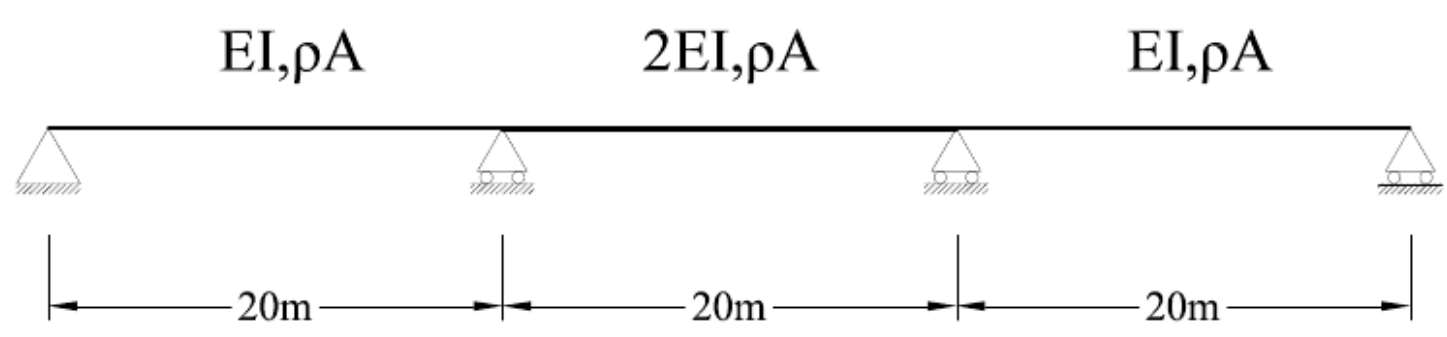

Figure 2.10: A three-span continuous stepped beam

\section{Envelopes determination:}

In this first case, it's determined the envelope curve of maximum accelerations on mid-span point of the central span by the proposed method. In this case, it's determined the response 
in several train velocities as well as the slopes $\left(\frac{d a}{d v}\right)$ so that a cubic spline can be interpolated by the analyzed points.

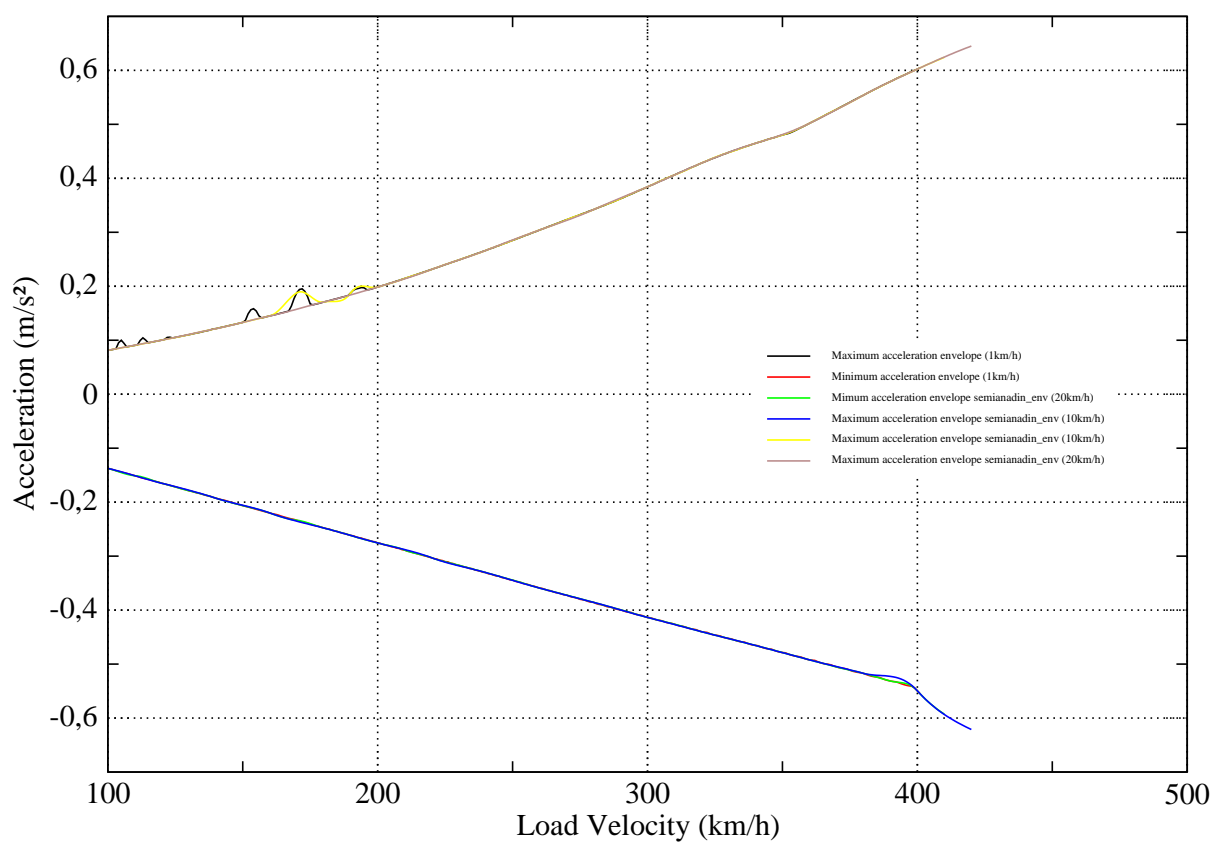

Figure 2.11: Max-Min Envelopes of test 2

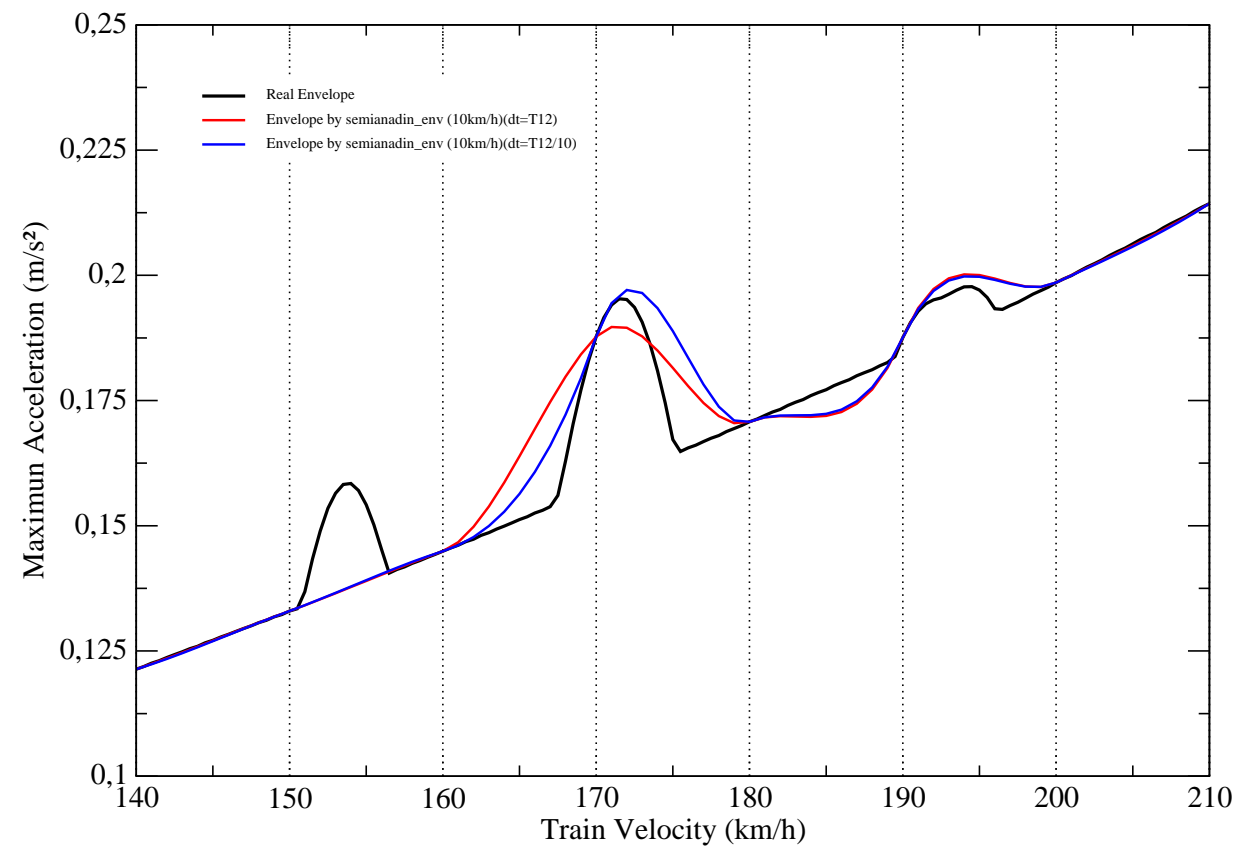

Figure 2.12: Detail of maximum acceleration test2

In this second test it's easy to check the sensibility of the solution to the time stepping. As it can be observed in the detail figure 2.12, the slope improves as the time stepping is smaller 
cause the gradient is approximated only by the variation to the train velocity, assuming that the variation respect to the time is zero cause the peaks of the time series are picked up to the envelope curve. However, it can't be ignored that this time stepping can't not get the exact time where this peak take place so as the time stepping gets smaller the approximation would be closer to the real time.

\section{Central Differences validation:}

In this second part of the test, the proposed method is validated by central difference derivatives. Hence, the response of the mid-span point of the central span is analyzed in terms of accelerations, two different load cases are considered, as well as different load velocities.

Case 1: one single load

In this first case one single load of $9.8 \mathrm{kN}$ is analyzed, starting from the origin of the structure. In order to analyze all the variables that influence the response, four velocities are chosen to study a wide range of slopes, three different modal combinations, one, three and twelve modes are considered so that pointing out the influence of the modal contributions in which frequency is the more relevant the more high is the derivation exponent, hence in acceleration, frequency contributes with a square value. Finally, because time series are analyzed with a time stepping, two different time steppings are taken into consideration, $\frac{T_{12}}{10}$ and $\frac{T_{12}}{100}$. All the results are summarized in table 2.1:

\begin{tabular}{|c|c|c|c|c|c|c|}
\hline \multirow[b]{2}{*}{$\begin{array}{c}\mathrm{v} \\
\mathrm{km} / \mathrm{h}\end{array}$} & \multicolumn{2}{|c|}{1 mode } & \multicolumn{2}{|c|}{3 modes } & \multicolumn{2}{|c|}{12 modes } \\
\hline & \multicolumn{2}{|c|}{$\mathrm{m} / \mathrm{s}^{2}$} & "(1)" & $\mathrm{m} / \mathrm{s}^{2}$ & "(1)" & $\mathrm{m} / \mathrm{s}^{2}$ \\
\hline 90 & $-9.18 \mathrm{E}-03$ & 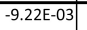 & $4.88 \mathrm{E}-03$ & $5.17 \mathrm{E}-03$ & $3.18 \mathrm{E}-03$ & $3.18 \mathrm{E}-03$ \\
\hline 130 & $2.30 \mathrm{E}-02$ & $2.30 \mathrm{E}-02$ & $1.95 \mathrm{E}-03$ & $1.95 \mathrm{E}-03$ & $3.73 \mathrm{E}-03$ & $3.73 \mathrm{E}-03$ \\
\hline 152 & $-1.63 \mathrm{E}-02$ & $-1.63 \mathrm{E}-02$ & $-5.71 E-03$ & $-5.70 \mathrm{E}-03$ & $6.47 \mathrm{E}-02$ & $6.59 \mathrm{E}-02$ \\
\hline 170 & $-9.55 E-03$ & $-9.56 \mathrm{E}-03$ & $1.18 \mathrm{E}-02$ & $1.18 \mathrm{E}-02$ & $3.52 \mathrm{E}-02$ & $3.57 E-02$ \\
\hline "(1)" & $\begin{array}{l}\text { Central Finite } \\
\qquad \Delta v=0 .\end{array}$ & $\begin{array}{l}\text { Difference } \\
\mathrm{km} / \mathrm{h}\end{array}$ & & & & \\
\hline "(2)" & $\frac{\partial a}{\partial v}+(l k /$ & & & & & \\
\hline
\end{tabular}

\begin{tabular}{|c|c|c|c|c|c|c|}
\hline \multirow{2}{*}{$\begin{array}{c}\mathrm{v} \\
\mathrm{km} / \mathrm{h}\end{array}$} & \multicolumn{2}{|c|}{1 mode } & \multicolumn{2}{|c|}{3 modes } & \multicolumn{2}{|c|}{12 modes } \\
\hline & "(1)" $\mathrm{m} / \mathrm{s}$ & "(2)" & "(1)" $\mathrm{m} / \mathrm{s}$ & "(2)" & "(1)" $\mathrm{m} / \mathrm{s}$ & "(2)" \\
\hline 90 & $-8.52 \mathrm{E}-03$ & $-8.56 \mathrm{E}-03$ & $-2.08 \mathrm{E}-04$ & $4.92 \mathrm{E}-05$ & $3.18 \mathrm{E}-03$ & $3.18 \mathrm{E}-03$ \\
\hline 130 & $2.30 \mathrm{E}-02$ & $2.30 \mathrm{E}-02$ & $1.95 \mathrm{E}-03$ & $1.95 \mathrm{E}-03$ & $3.73 E-03$ & $3.73 E-03$ \\
\hline 152 & $-1.57 \mathrm{E}-02$ & $-1.57 E-02$ & $-8.69 \mathrm{E}-03$ & $-8.68 \mathrm{E}-03$ & $3.43 \mathrm{E}-02$ & $3.52 E-02$ \\
\hline 170 & $-9.89 \mathrm{E}-03$ & $-9.90 E-03$ & $1.25 \mathrm{E}-02$ & $1.25 \mathrm{E}-02$ & $2.96 \mathrm{E}-02$ & $3.01 E-02$ \\
\hline
\end{tabular}

Table 2.1: One single load central differences analysis 


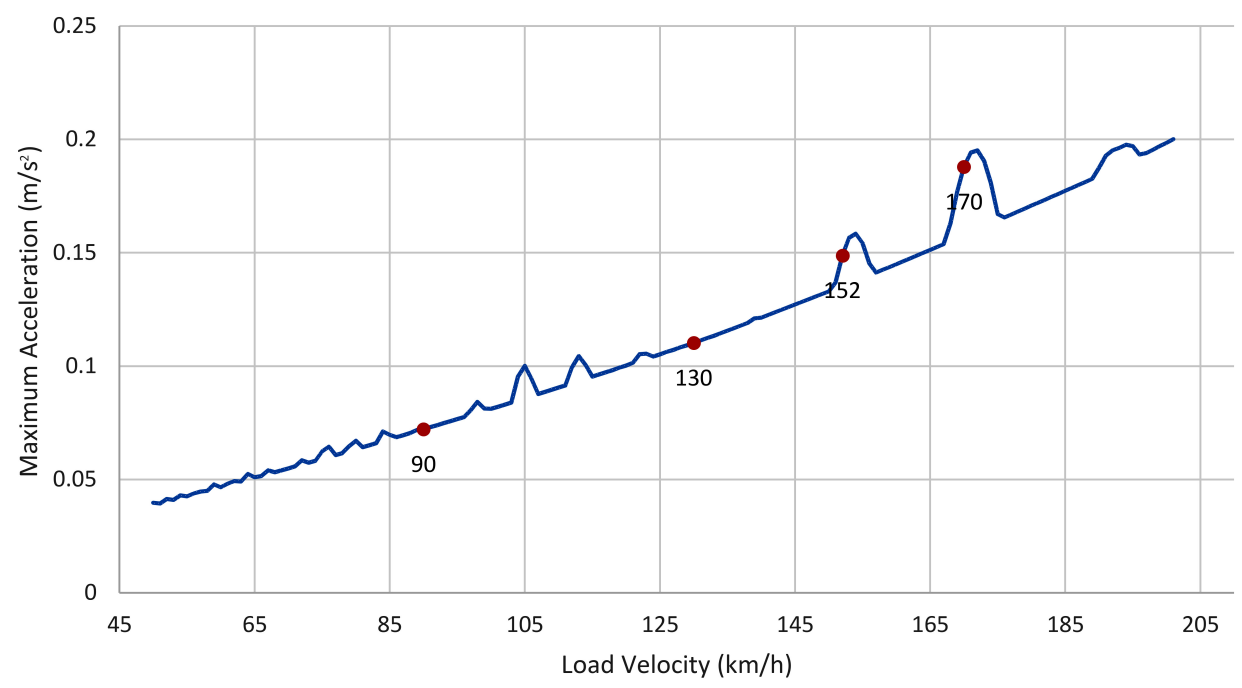

Figure 2.13: Acceleration envelope and analyzed velocities

Case 2: two load

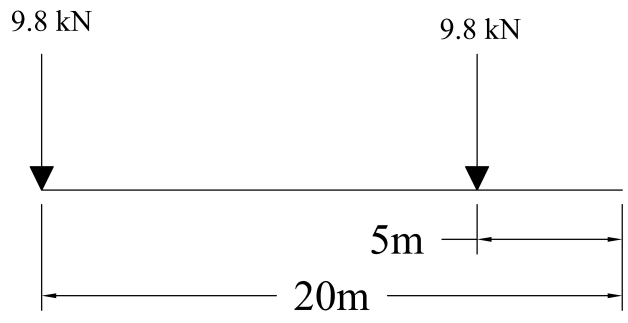

Figure 2.14: Load case 1: Two point loads.

In this second case, two point loads are considered to value the effects of load superposition, besides, the first case is positioned $5 \mathrm{~m}$ from structure's origin so that analyze the dead time before the load gets into the structure on derivate expression. Exactly as latter case, four velocities, three modal combinations and two time steppings are studied, information that is one again summarized in the following table 2.2:

\begin{tabular}{|c|c|c|c|c|c|c|}
\hline \multirow[b]{2}{*}{$\begin{array}{c}\mathrm{v} \\
\mathrm{km} / \mathrm{h}\end{array}$} & \multicolumn{2}{|c|}{1 mode } & \multicolumn{2}{|c|}{3 modes } & \multicolumn{2}{|c|}{12 modes } \\
\hline & "(1)" & "(2)" & "(1)" & "(2)" & "(1)" $m /$ & "(2)" \\
\hline 90 & $1.37 \mathrm{E}-03$ & $1.37 \mathrm{E}-03$ & $2.81 \mathrm{E}-03$ & $\begin{array}{ll}2.81 \mathrm{E}-03 \\
\end{array}$ & $4.38 \mathrm{E}-04$ & $1.62 \mathrm{E}-04$ \\
\hline 130 & $1.09 \mathrm{E}-02$ & $1.09 \mathrm{E}-02$ & $1.48 \mathrm{E}-03$ & $1.48 \mathrm{E}-03$ & $1.64 \mathrm{E}-02$ & $1.64 \mathrm{E}-02$ \\
\hline 152 & $1.05 \mathrm{E}-03$ & $1.05 \mathrm{E}-03$ & $2.28 \mathrm{E}-03$ & $2.28 \mathrm{E}-03$ & $-2.05 E-03$ & $-8.37 \mathrm{E}-03$ \\
\hline 170 & $-3.39 E-02$ & $-3.39 \mathrm{E}-02$ & $-3.44 \mathrm{E}-02$ & $-3.44 E-02$ & $-4.30 E-02$ & $-4.37 E-02$ \\
\hline "(1)" & $\begin{array}{r}\text { ntral Finit } \\
\Delta v\end{array}$ & $\begin{array}{l}\text { ifference } \\
\mathrm{km} / \mathrm{h}\end{array}$ & & & & \\
\hline "(2)" & $\frac{\partial a}{\partial v}+(l k)$ & $\cdot \partial a / \partial t$ & & & & \\
\hline
\end{tabular}

\begin{tabular}{|c|c|c|c|c|c|c|}
\hline \multirow[b]{2}{*}{$\begin{array}{c}\mathrm{v} \\
\mathrm{km} / \mathrm{h}\end{array}$} & \multicolumn{2}{|c|}{1 mode } & \multicolumn{2}{|c|}{3 modes } & \multicolumn{2}{|c|}{12 modes } \\
\hline & "(1)" $\mathrm{m} / \mathrm{s}$ & & "(1)" & "(2)" & "(1)" & "(2)" \\
\hline 90 & $1.17 \mathrm{E}-03$ & $1.17 \mathrm{E}-03$ & $1.85 \mathrm{E}-03$ & $1.86 \mathrm{E}-03$ & $2.81 \mathrm{E}-03$ & $2.59 \mathrm{E}-0 \mathrm{z}$ \\
\hline 130 & $1.09 \mathrm{E}-02$ & $1.09 \mathrm{E}-02$ & $1.96 \mathrm{E}-03$ & $1.96 \mathrm{E}-03$ & $6.77 \mathrm{E}-03$ & $6.63 \mathrm{E}-03$ \\
\hline 152 & $1.10 \mathrm{E}-03$ & $1.10 \mathrm{E}-03$ & $1.95 \mathrm{E}-03$ & $1.95 \mathrm{E}-03$ & $-3.03 E-03$ & $-3.08 \mathrm{E}-03$ \\
\hline 170 & $-3.45 \mathrm{E}-02$ & $-3.45 E-02$ & $-3.80 \mathrm{E}-02$ & $-3.80 \mathrm{E}-02$ & $-4.70 \mathrm{E}-02$ & $-4.77 \mathrm{E}-02$ \\
\hline
\end{tabular}

Table 2.2: Two loads central differences analysis. 


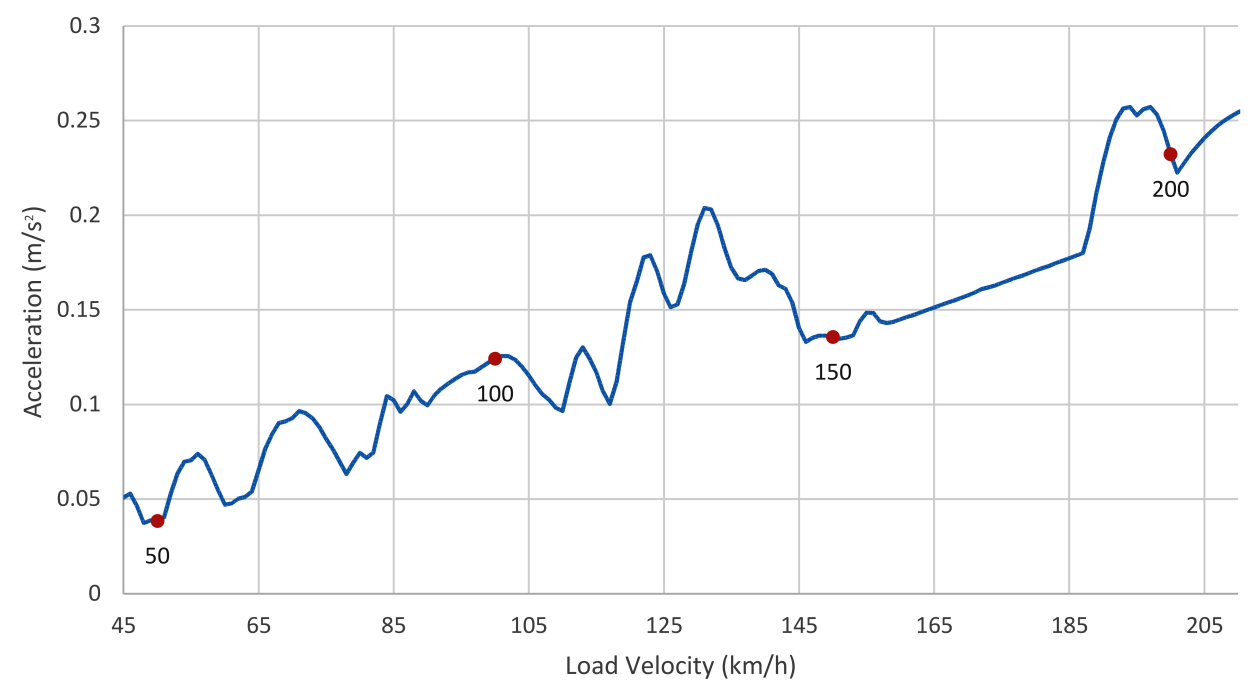

Figure 2.15: Acceleration envelope and analyzed velocities

\subsubsection{Three-span bridge according to Eurocode 1.}

The purpose of this new test is to illustrate the application of this new method to a real highspeed bridge. Particularly, the Eurocode 1 analyzes a real three-span high-speed bridge, with lateral spans of $25 \mathrm{~m}$ and a central one with $38 \mathrm{~m}$. The cross-section per length is $\rho A$ is $14435.25 \mathrm{~kg} / \mathrm{m}$, flexural stiffness EI $110649.6 \mathrm{MNm}^{2}$, constant along all the structure. The damping ration $\xi$ is taken as 0.01 for all modes of vibration. This bridge, according to Eurocode 1 , is analyzed under the action of the HSML model. Once again, the modal analysis is carried out on SAP2000, modeling each span by ten frame elements. The modal information is illustrated in the table 2.16:

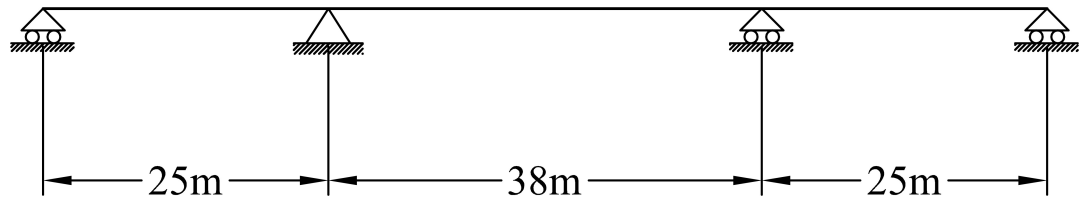

Figure 2.16: Three-span bridge according to Eurocode 1.

\begin{tabular}{|c|c|}
\hline Mode of vibration & Frequency $(\mathrm{Hz})$ \\
\hline \hline 1 & 4.28 \\
2 & 8.20 \\
3 & 9.59 \\
4 & 10.98 \\
5 & 15.81 \\
\hline
\end{tabular}

Table 2.3: Modes and frequencies used in three-span brdige according to Eurocode 1. 
Firstly, a regular analysis by semianalytic methodology is carried out from $144 \mathrm{~km} / \mathrm{h}$ to $422 \mathrm{~km} / \mathrm{h}, \mathrm{km} / \mathrm{h}$ per $\mathrm{km} / \mathrm{h}$. Time stepping is fixed to $0.0063 \mathrm{~s}$, as well as a residual time to six times the highest period of the structure and the ten HSML trains are studied. Then, the structure is analysixzed by the proposed methodology in the exact same conditions but a velocity step of $10 \mathrm{~km} / \mathrm{h}$. The results obtained are summarized in the following graphs:

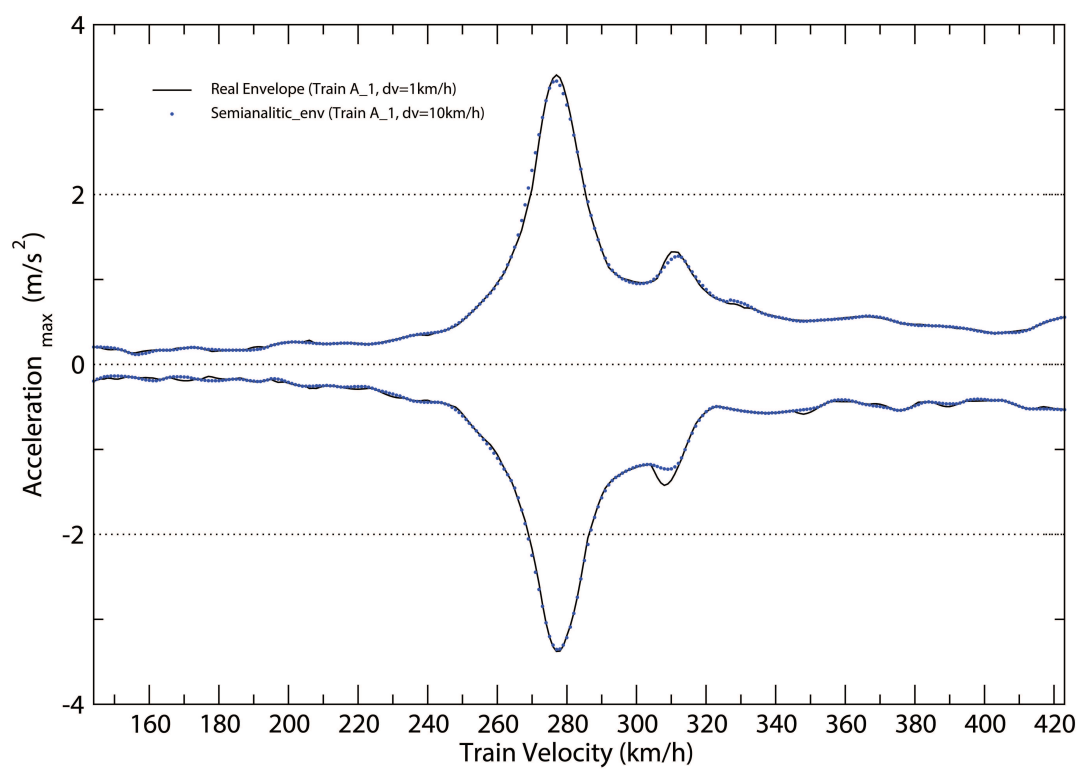

Figure 2.17: Acceleration Envelope. Train $A_{1}$ 


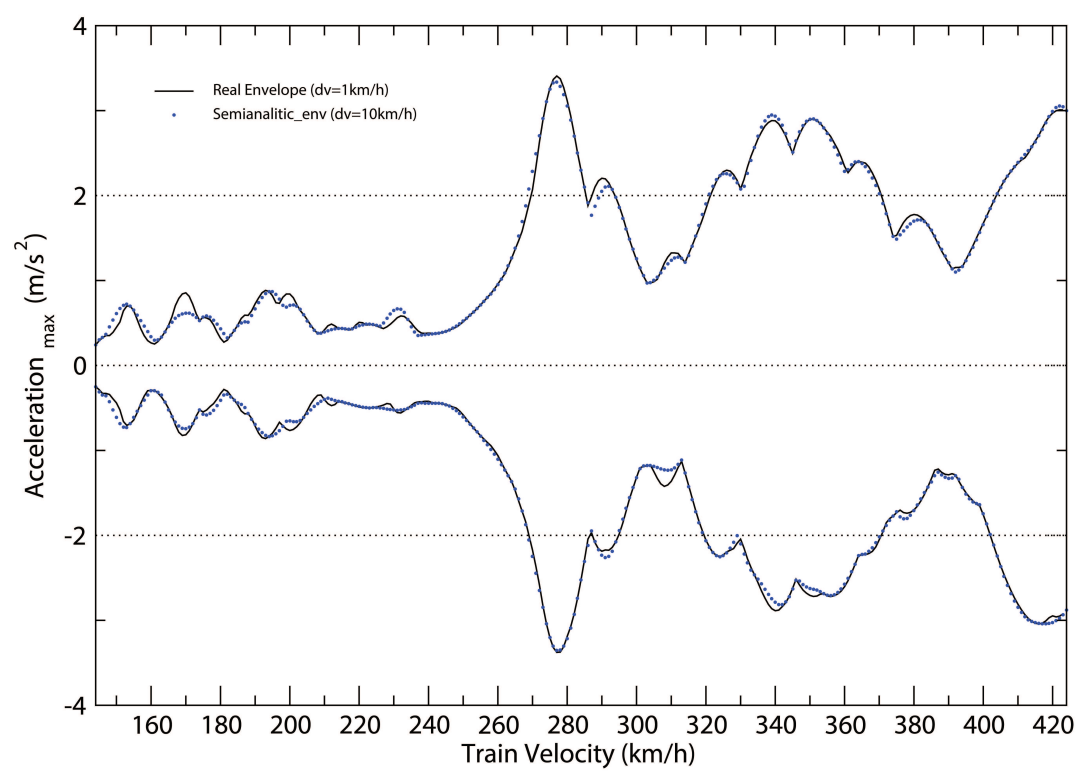

Figure 2.18: Acceleration Envelope. All trains.

Then, the effects of the two principal variables, time stepping and velocity steps, are analyzed. Firstly, in the same conditions, the structure is analyzed by two different velocity steps, 10 and $20 \mathrm{~km} / \mathrm{h}$ per $\mathrm{km} / \mathrm{h}$. The results in terms of absolute values 2.19 : 


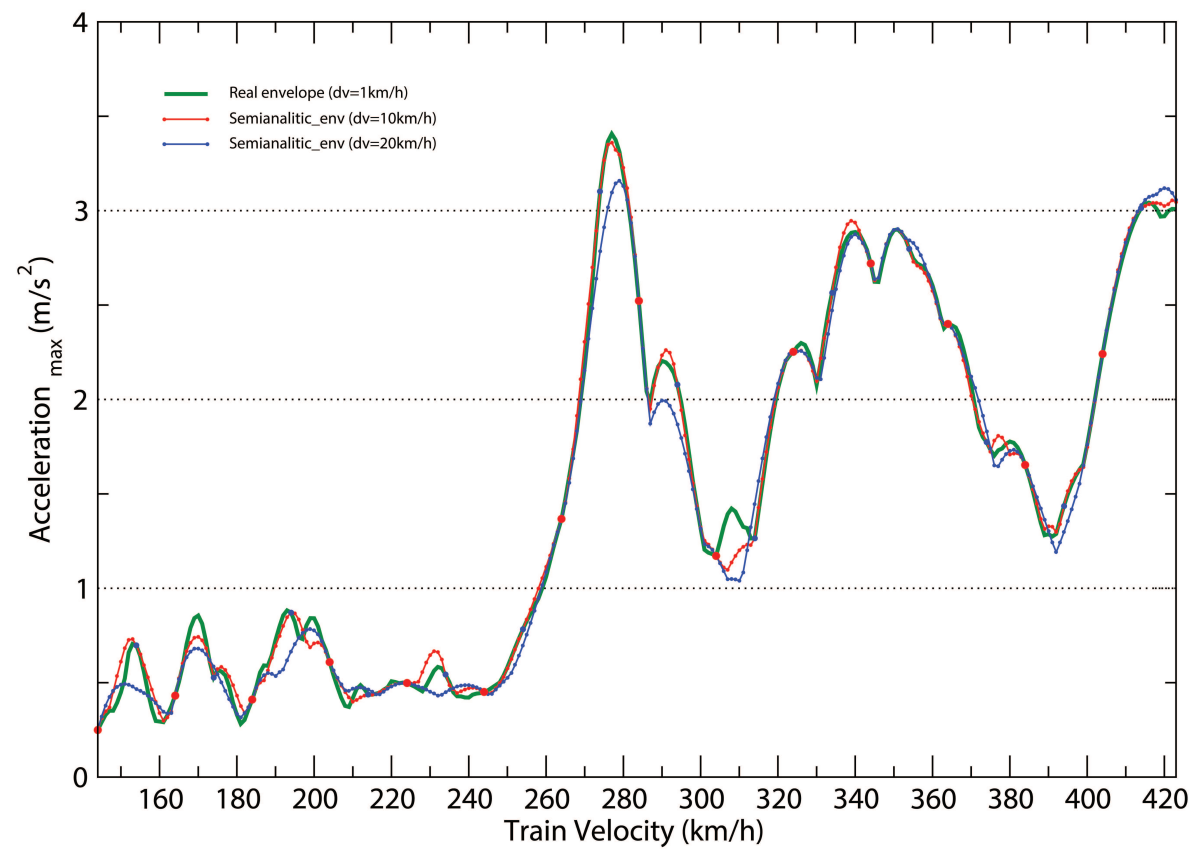

Figure 2.19: Maximum Absolute Acceleration with velocity steps 10 and $20 \mathrm{~km} / \mathrm{h}$ per km/h

Associated with the latter graph, it's interesting to analyze the errors of the cubic interpolation by the relative percentage error 2.20 : 


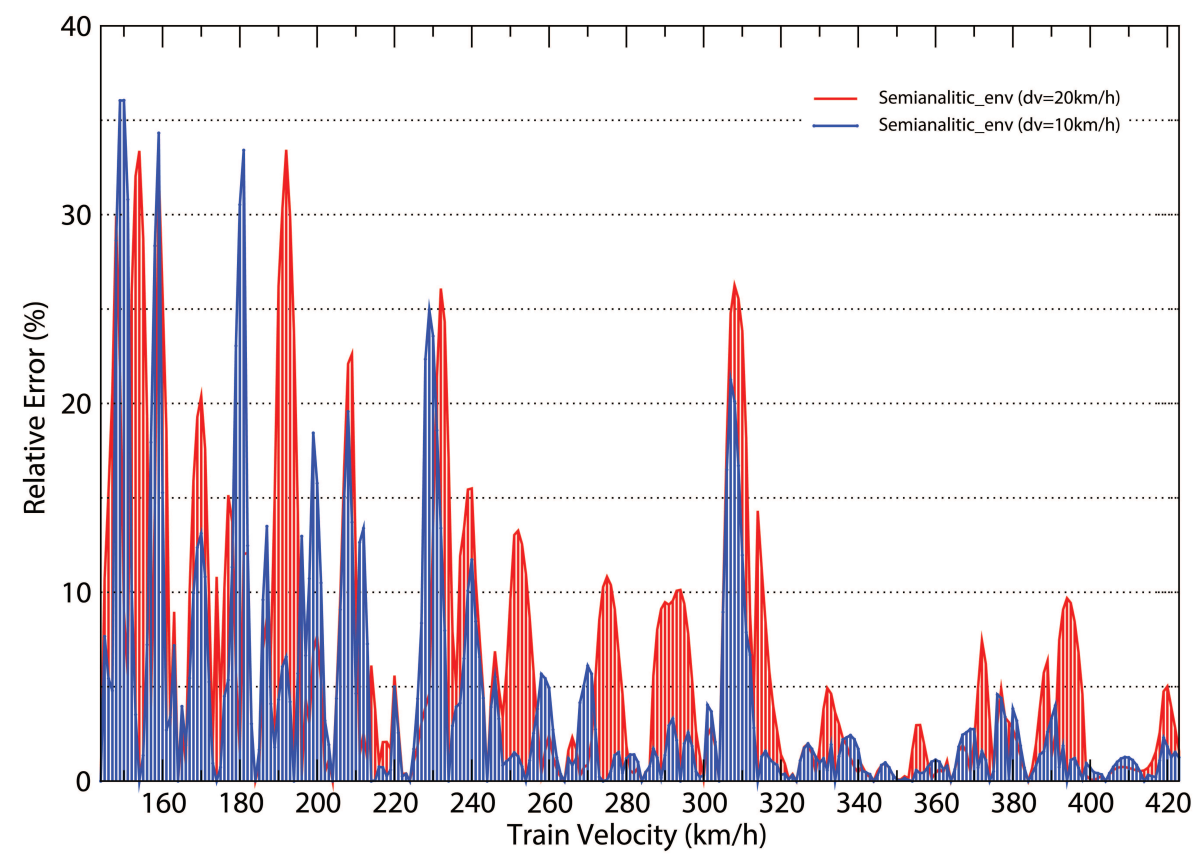

Figure 2.20: Relative Errors in Maximum acceleration.

Finally, the effects of time stepping lies on the detection of the peaks on the acceleration series, hence the smaller it's defined the smaller relative errors should be obtained. Hence, to analyze this effect, two different time stepping are defined, $0.006 \mathrm{~s}$ and $0.001 \mathrm{~s}$, and the relative errors are determined as before, 2.21: 

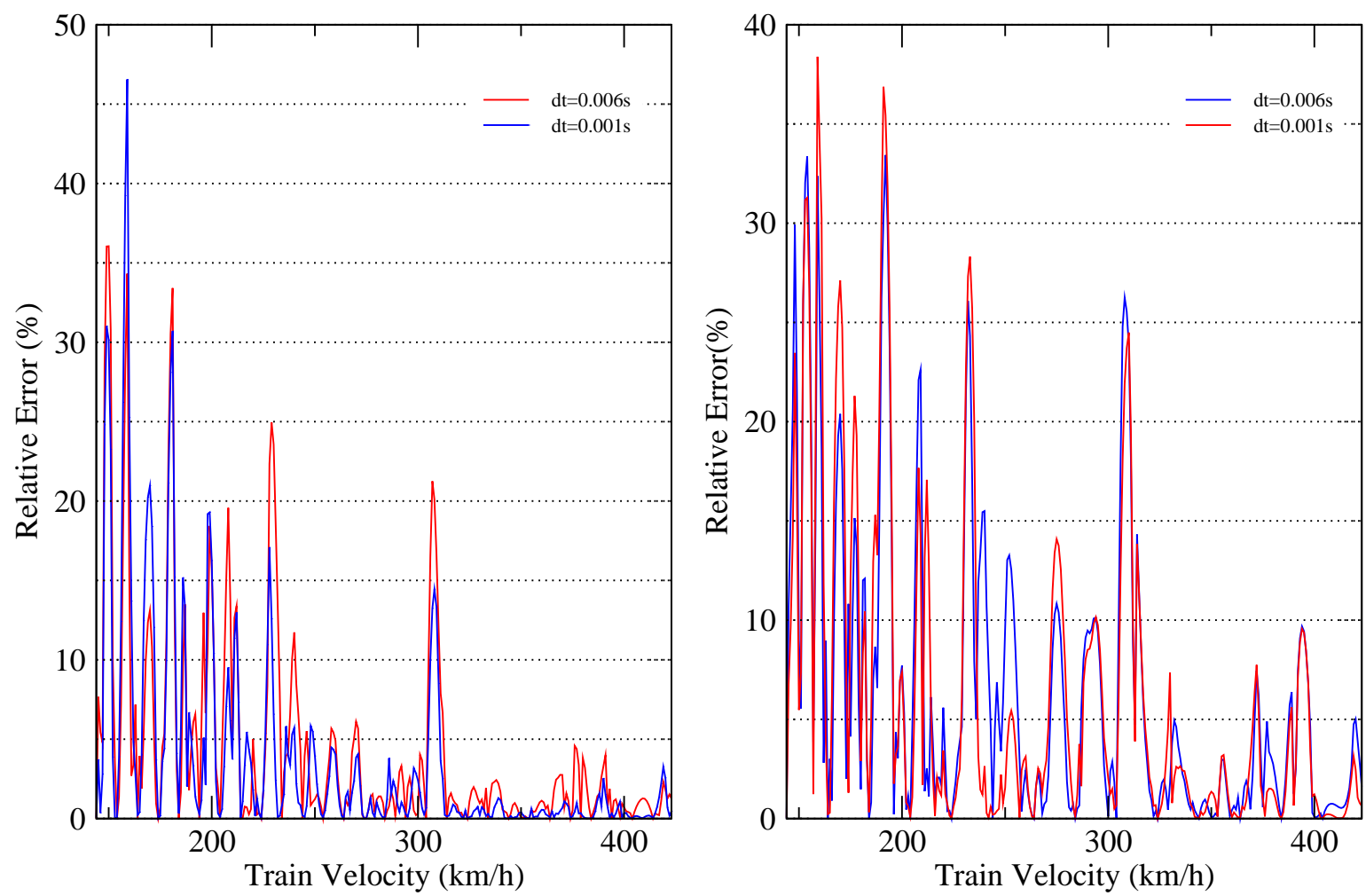

Figure 2.21: Relative errors associated to two different velocity steps, 10 and $20 \mathrm{~km} / \mathrm{h}$ per $\mathrm{km} / \mathrm{h}$.

\subsubsection{Bowstring steel-concrete composite bridge}

The last three tests are tridimensional bridges. This first test is taken from the final degree project [45], in which the author developed a methodology of comparison between the most common variables of steel-concrete composite bowstring arch bridges in terms of dynamic response and economy. In this reference, all the dynamic analysis were carried out with the Semi-Analytic solution and the built FEM model is rescued to be analyzed by this new $\mathrm{dq} / \mathrm{dv}$ method.

\section{Description of the structure}

The structure is composed by five span 25-30-80-25-30 bridge, with a parabolic arc of $12 \mathrm{~m}$ height with a relationship width-height in the $30 \mathrm{~m}$ spans of $\frac{30}{2.6}=11.53$, typical in railway bridges. In the central span this relations reaches a value of $\frac{80}{2.6}=30.77$ ", quite similar to Santa Ana bridge placed in the high-speed line of Córdoba-Málaga, $\frac{86.4}{2.6}=33.23$, steel-concrete composite bridge of similar characteristics.

The deck is designed for a single rail of $6 \mathrm{~m}$, with $2.74 \mathrm{~m}$ of sidewalk on both laterals, with a total width of $11.47 \mathrm{~m}$. 
With respect to the arc, it's designed in a rectangular box made of S355 steel, with $2 \mathrm{~mm}$ thickness, full with concrete HA-30 in order to increase the resistance to vertical buckling and obtain a higher dynamic damping. Each arc is tied by 9 cables, separated $8 \mathrm{~m}$, with circular section of 40mm diameter and steel ST1570.

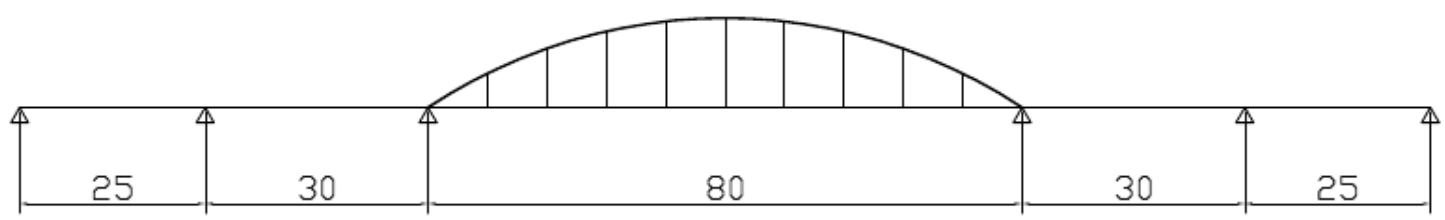

Figure 2.22: Elevation view.

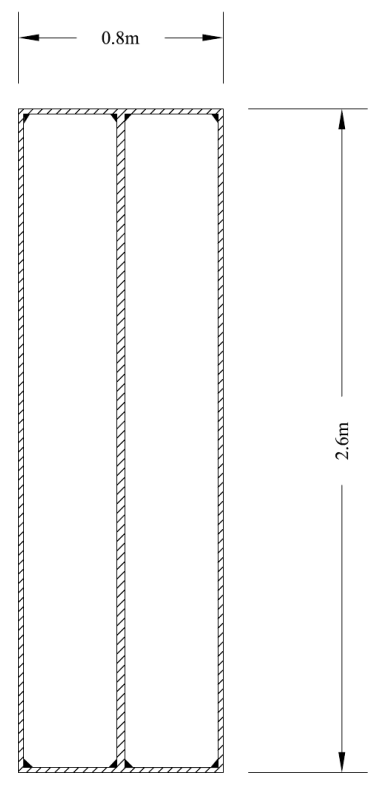

Figure 2.23: Cross section of lateral beams.

\section{FEM model}

In the FEM model the two main beams are defined 8 degrees inclined to the rails, defined by thick shell elements. The arc is modeled with 3D frame elements, also inclined 18 degrees to the rail and with 8 tubular bracings with a diameter of $800 \mathrm{~mm}$ and a thickness of $20 \mathrm{~mm}$ defined through a buckling ultimate limit state. In reference to the concrete deck, it is modeled with shell elements resting on lateral beams through shear studs modeled as infinite rigid beams and taking into consideration the eccentricity of both elements so that the 
deck is defined in the projected elevation. These lateral beams, with variable section as it can be seen in figure 2.26, are discretely modeled with frame elements because their main function is to share out the flexion effort, hence they are modeled in three constant parts, with sections given in figure 2.26 , and lengths of $2.414 \mathrm{~m}, 6.750 \mathrm{~m}$ y $2.414 \mathrm{~m}$. These lateral beams are set each $3.13 \mathrm{~m}$ in the span of $25 \mathrm{~m}, 3.75 \mathrm{~m}$ in the span of $30 \mathrm{~m}$ and, finally, eah $4 \mathrm{~m}$ in the central span. In the other hand, a ribbed panel is defined supporting the concrete deck, so in the FEM model, it's influence is defined through the mechanic properties of the concrete deck with an increment of $2 \%$ in the membrane rigidity and a $5 \%$ in bending rigidity in the longitudinal direction, through a mechanical analysis in the behaviur of this two elements acting simultaneously. A reduction in the mass of the concrete deck is also taken into consideration. Finally, shear studs are considered in the joints between the main longitudinal and the lateral beams in the lateral spans as well as under the cables and between them in the tied-arc span. In the same way, the encounters arc-deck are stiffened with horizontal plate stiffeners between two adjacent stiffeners, avoiding by this way fictitious modes of the encounter between the arc and the deck.

Through all the latter considerations, it results the model represented in figures 2.24 and 2.25:

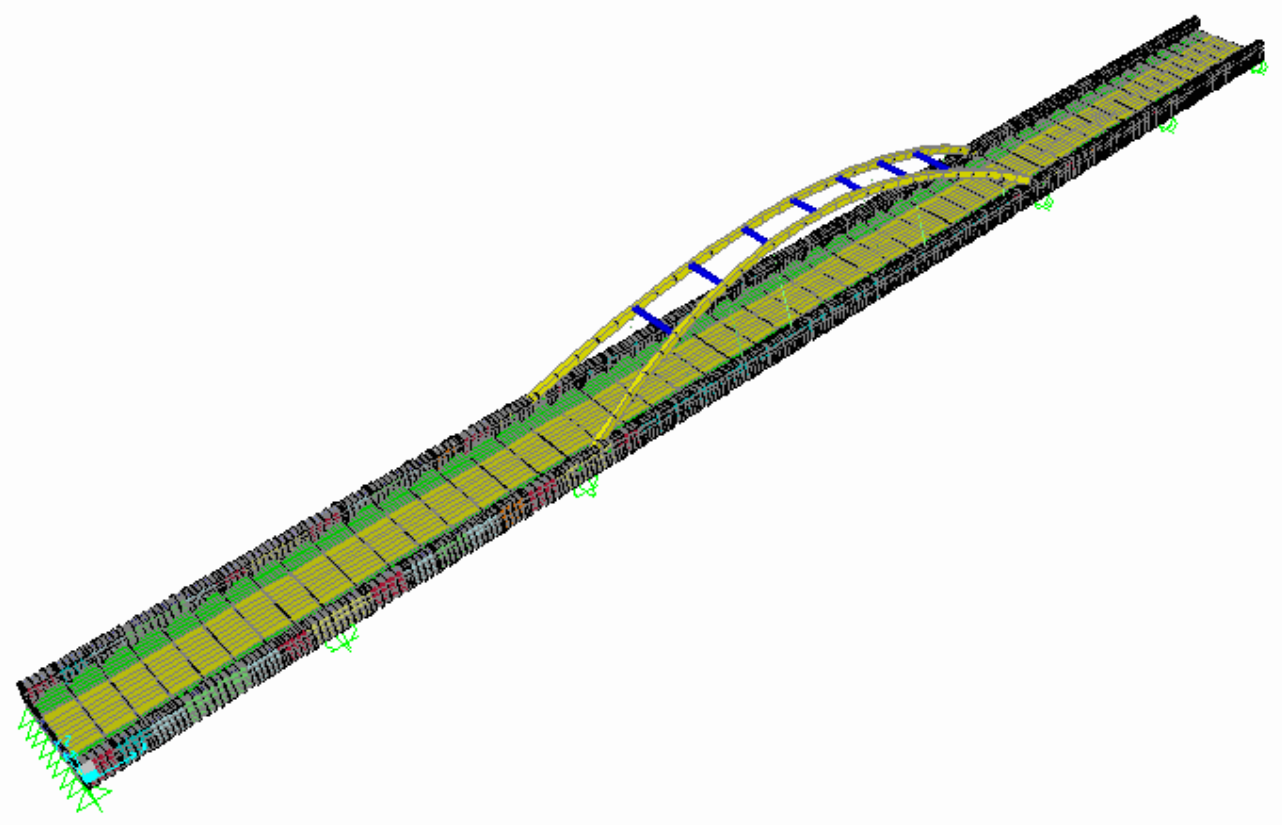

Figure 2.24: Tridimensional FEM. 


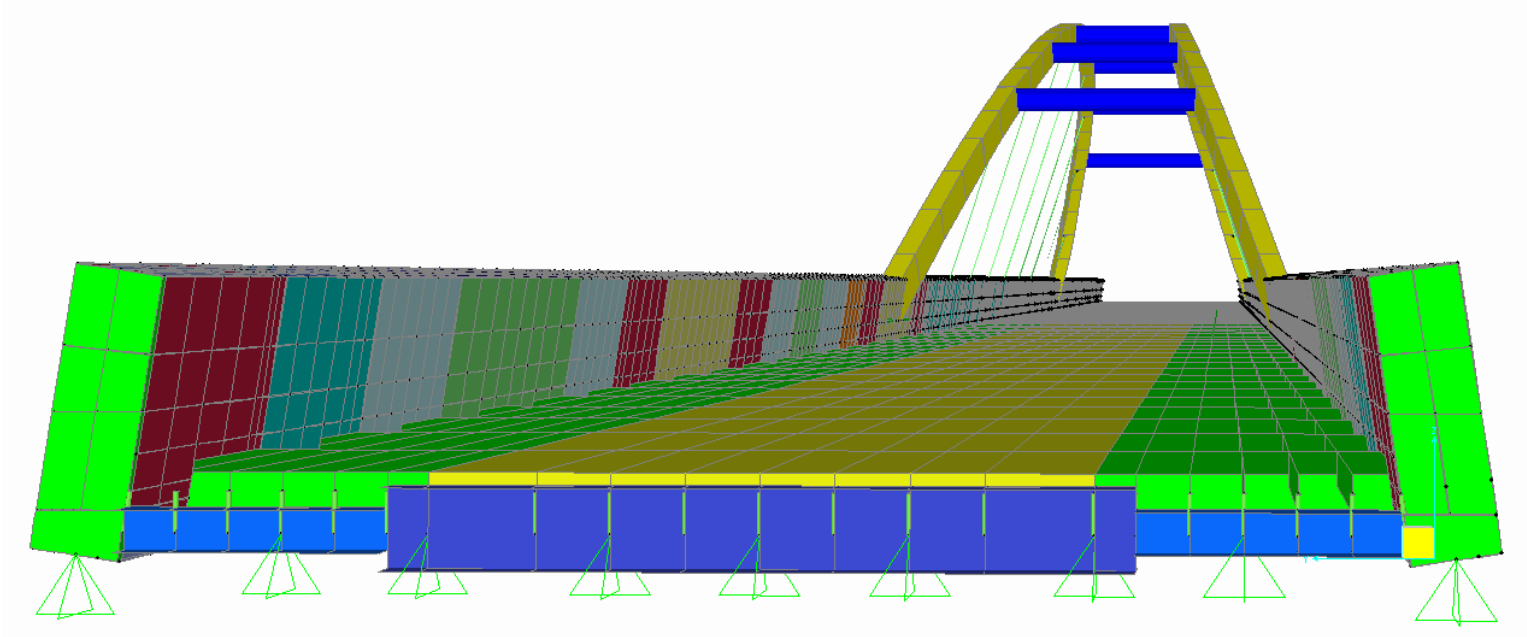

Figure 2.25: Detail of lateral beams and the joint with the concrete deck.

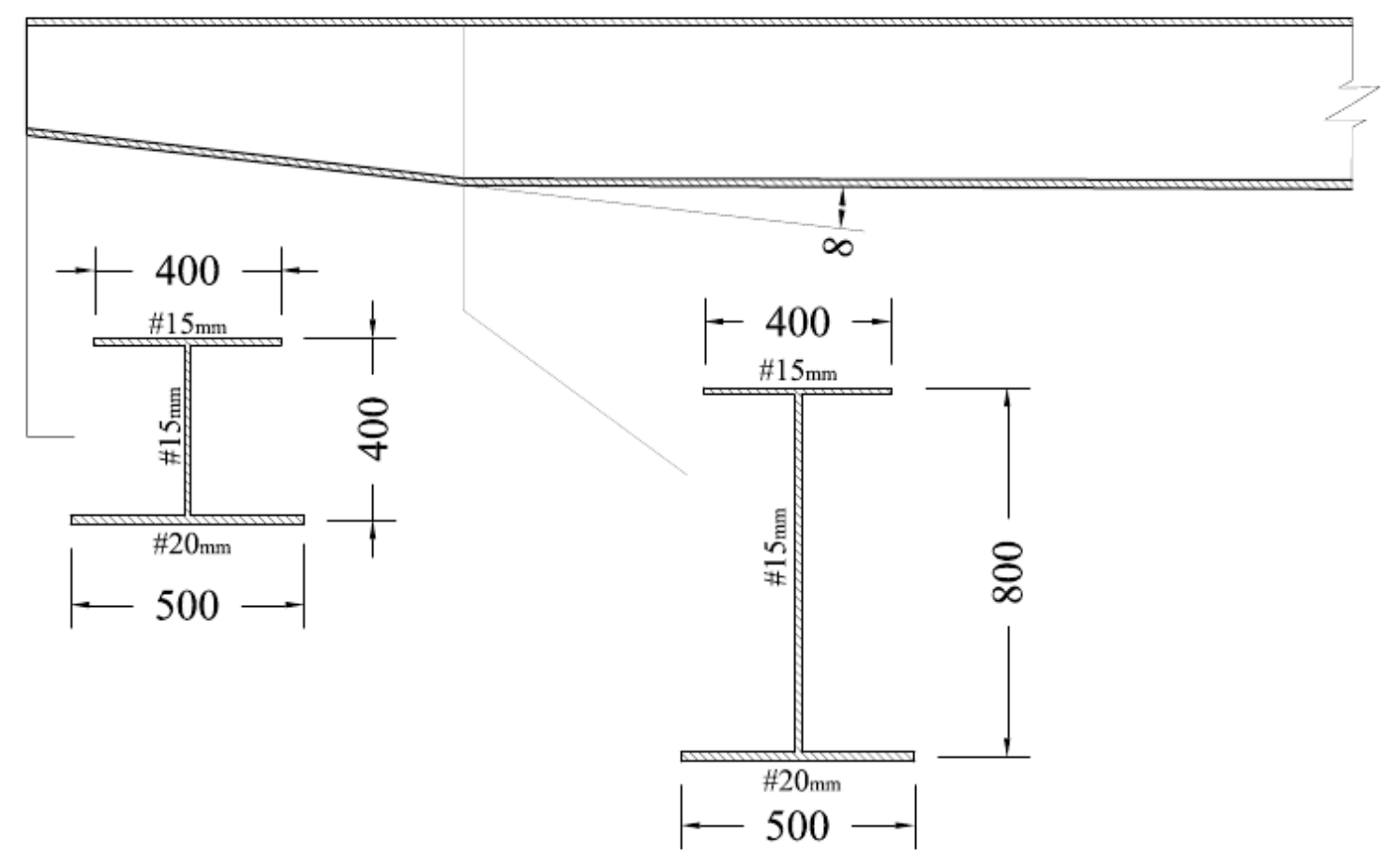

Figure 2.26: Transversal variable height beams detail. 


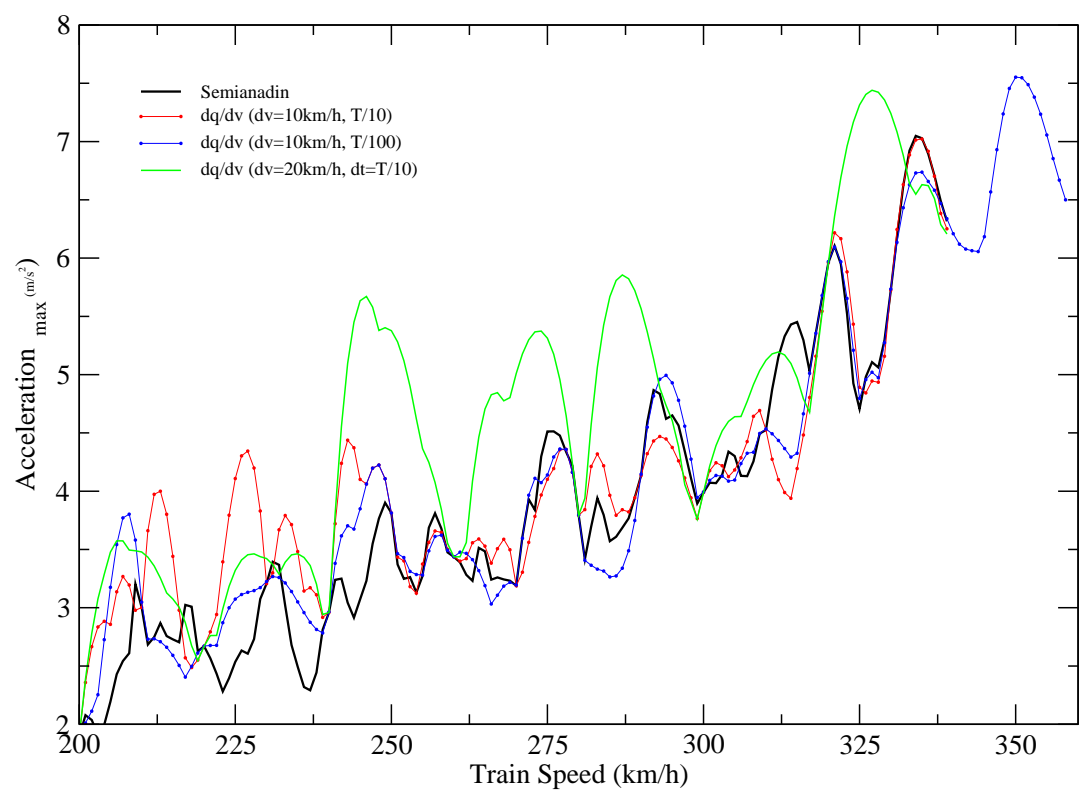

Figure 2.27: Acceleration envelope in mid central span under train A1 of HSML series

Dynamic analysis: maximum displacement envelope

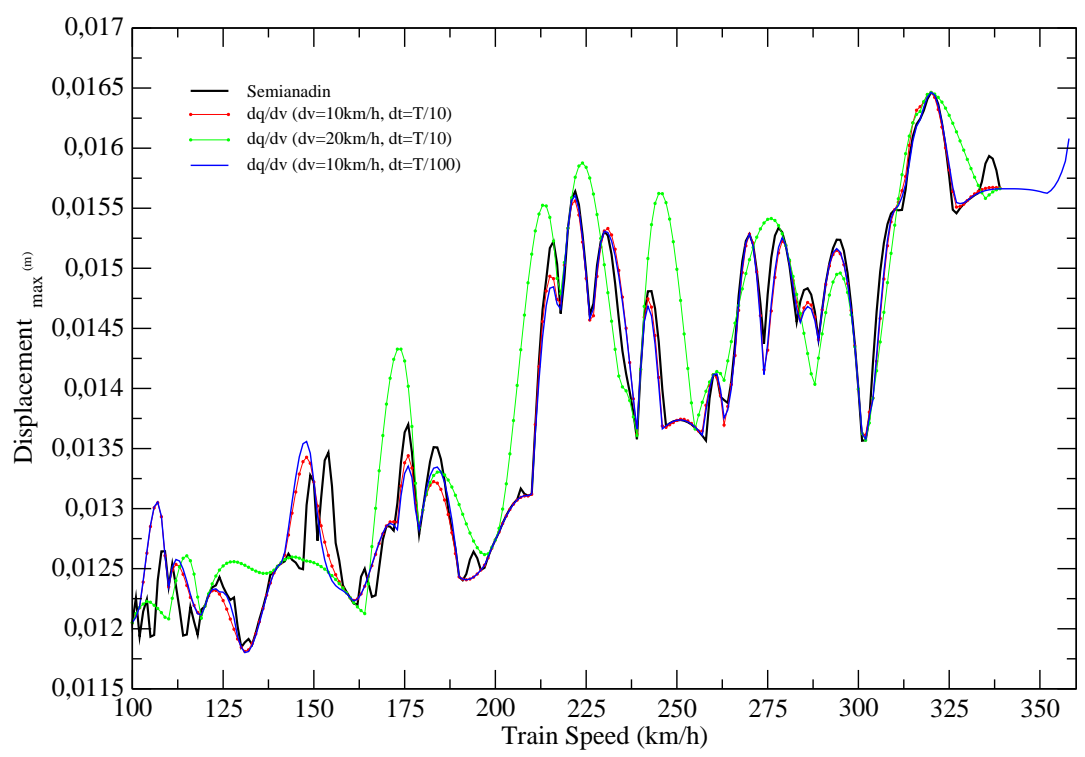

Figure 2.28: Displacement envelope in mid central span under train A1 of HSML series 


\section{Conclusion}

The results are not as good as expected, therefore, to obtain acceptable results in the envelope analysis it is necessary to define short time steppings what reduces the improvements in time of calculus. The guilty of this bad behavior is the definition of the load line, as retaken from the mentioned document, the original FEM model did not considered any boundary in the rails in their connection with the adjacent structures. This supposes a sharp transition for the loads what is translated in a impulsive force. As mentioned in the introduction, this methodology is very sensitive to frequency because the derivative is proportional to the square value of it, besides, impulsive forces are characterized by a constant Fourier transformation exciting all the frequencies. This fact makes the solution very fluctuating, thus, to obtain accurate results it would oblige to define very short time steps for which this proposed methodology is not competitive. Nevertheless, this effect is fictitious because in real structures the rails are continuous and secondary elements as the ballast impose boundary conditions. For Semi-analytic solution is not an important issue because of its formulation which highly reduces this fluctuating behaviors, at the contrary, $\mathrm{dq} / \mathrm{dv}$ solution is highly sensitive for this behavior this more accurate modelization is needed because to obtain competitive results.

In the following two tests the rails at the endings are fixed in vertical translation and rotation around the transverse direction in order to simulate the continuity of the rails the connection of the structure to adjacent ones, with this it will be analyzed the actual competitive of the $\mathrm{dq} / \mathrm{dv}$ method once the fictitious impulsive excitations will be mitigated.

\subsubsection{Concrete box girder bridge. Rodenillo bridge.}

In this new test, a double track U-shaped girder bridge is analyzed by the proposed methodology in order to observe the influence of torsional modes in the results.

Description of the structure

It is defined a concrete box girder with a total length of $207 \mathrm{~m}$, composed by two lateral spans of $36 \mathrm{~m}$ and the rest of $45 \mathrm{~m}$ length. As shown in figure 2.29, the U girder has a height of $2.5 \mathrm{~m}$, a thickness of the bottom flange of $0.3 \mathrm{~m}, 0.5 \mathrm{~m}$ for the lateral webs and it supports a concrete deck of $14 \mathrm{~m}$ and $0.35 \mathrm{~m}$ thick. Furthermore, the box is reinforced by two $0.5 \mathrm{~m}$ thick diaphragms in its encounters with the bearings. A ballast layer of $50 \mathrm{~cm}$ thick lays on the deck as well as the UIC60 rails which are supported at every $0.6 \mathrm{~m}$ distance by prestressed concrete sleepers.

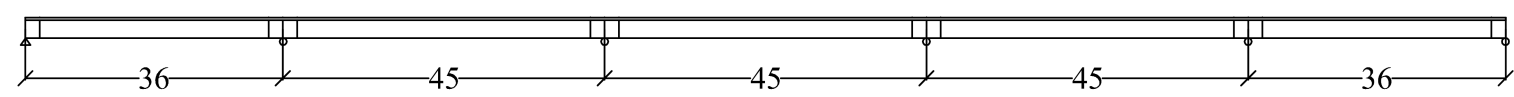

Figure 2.29: Lateral view. 


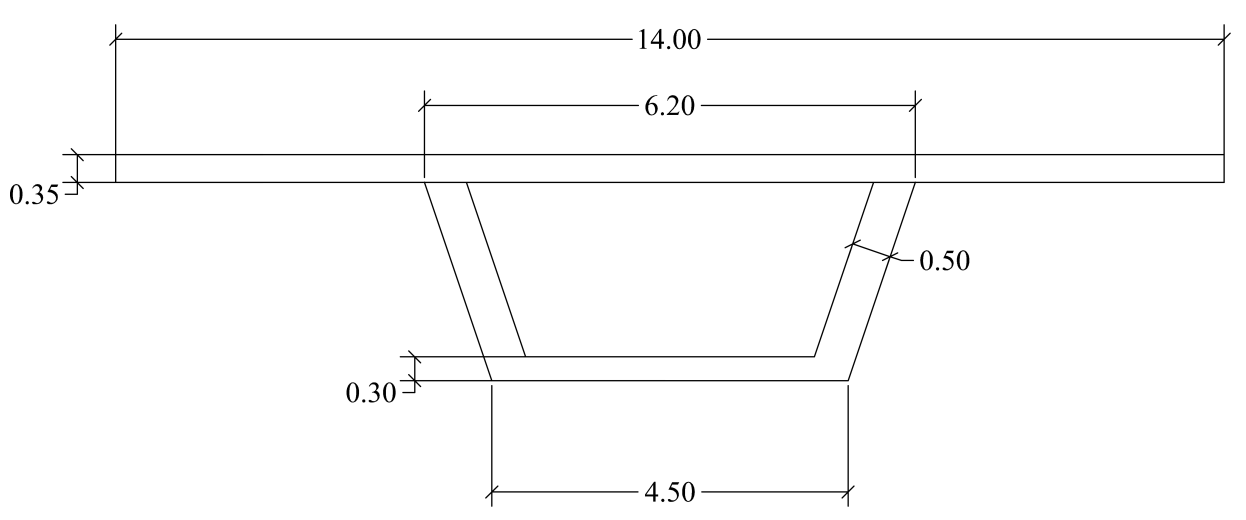

Figure 2.30: Cross section

\section{FEM model}

The bridge is modeled with 38 frames and 7422 shells on SAP2000v10 as shown in figure 2.31. The shells are defined with six sections: webs of $0.5 \mathrm{~m}$, top flange of $0.3 \mathrm{~m}$, bottom flange of $0.35 \mathrm{~m}$, diaphragms of $0.5 \mathrm{~m}$ and two different segments of the deck of $0.35 \mathrm{~m}$ thick. On the other hand, the barriers are modeled by frame elements. In order to simulate the effect of the increased reinforcement in the concrete deck through its transversal direction, the beam rigidity of the concrete deck is increased by $20 \%$, a good approximation in experience. The boundaries are the common in a bridge of simply supported spans, translational fixed bearings in one side and longitudinal fixed movement in the rest of supports. Furthermore, as mentioned in the previous test, the longitudinal movement and the rotation are fixed in the ends of the rails in order to simulate the connection with the adjacent lines. Whit the latter consideration, it is defined a smooth load line to be used in the following dynamic analysis with a mitigation of the fictitious impact effect at the entrance of the trains. The masses and the properties of the employed materials are summarized in the table 2.4:

\begin{tabular}{lll}
\hline Item & Unit & Value \\
\hline Per-unit-length mass of rail & $\mathrm{kgf} / \mathrm{m}$ & 60 \\
Mass of sleepers & $\mathrm{kg}$ & 290 \\
Young's modulus & $\mathrm{Mpa}$ & 35000 \\
Possion's ratio & - & 0.2 \\
Density & $\mathrm{kg} / \mathrm{m3}$ & 2403 \\
\hline
\end{tabular}

Table 2.4: Masses and properties of elements employed in U-Girder modelç 


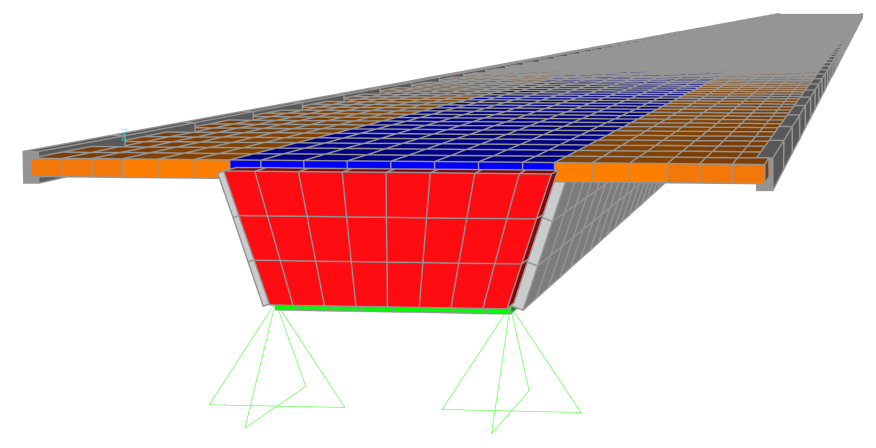

Figure 2.31: FEM model

Figure 2.32: FEM 3D model

Modal Analysis.

Modal analysis is carried out on the software SAP2000v.10. Attending to the Spanish standard IAPF-07, in the dynamic analysis, all the natural modes of vibration with a frequency under $30 \mathrm{~Hz}$ must be taken into the calculus, in this case, a total of 123 modes have been analyzed. The first two bending and the first torsional modes are represented in figure 2.33:

Dynamic analysis

A dynamic analysis of the above defined structure is carried out with the Semi-Analytic solution and the proposed methodology in order to validate the results. For all the analysis, the ten trains of the HSML series would be introduced in the calculus and the analysis would be developed during the train passage and six times the mayor period of vibration in order to analyze the behavior of the structure in free vibration. The response is obtained in 12 


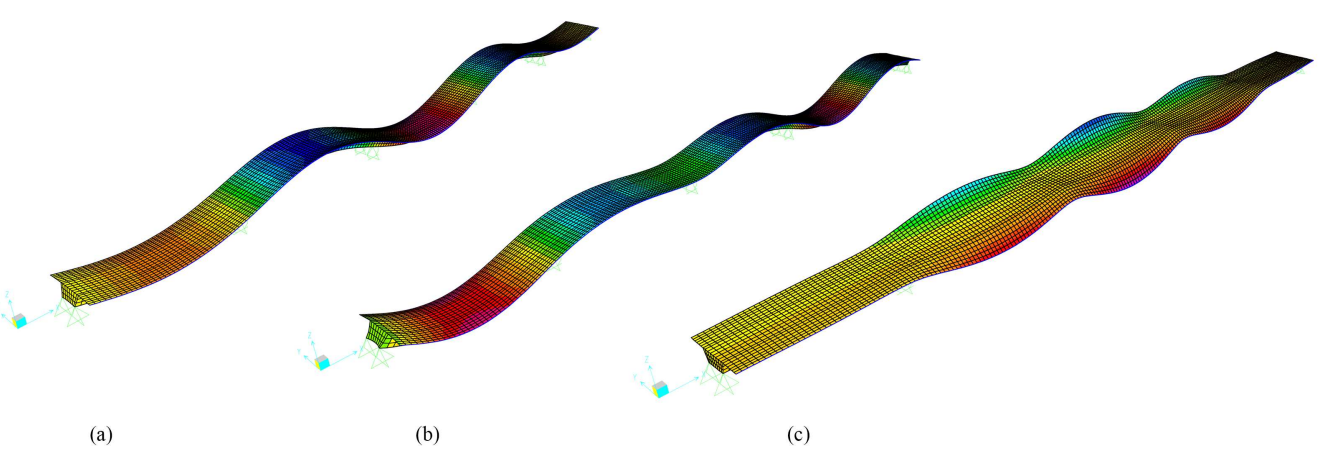

(a)

(b)

(c)

Figure 2.33: Natural modes. (a) first bending mode $2.58 \mathrm{~Hz}$, (b) second bending mode $2.93 \mathrm{~Hz}$, (c) first torsional mode $6.24 \mathrm{~Hz}$

post-process points. As can be seen in figure 2.34, the post-points for acceleration are placed in the center and in the edge of the ballast for the mid and the quarter length of the span.

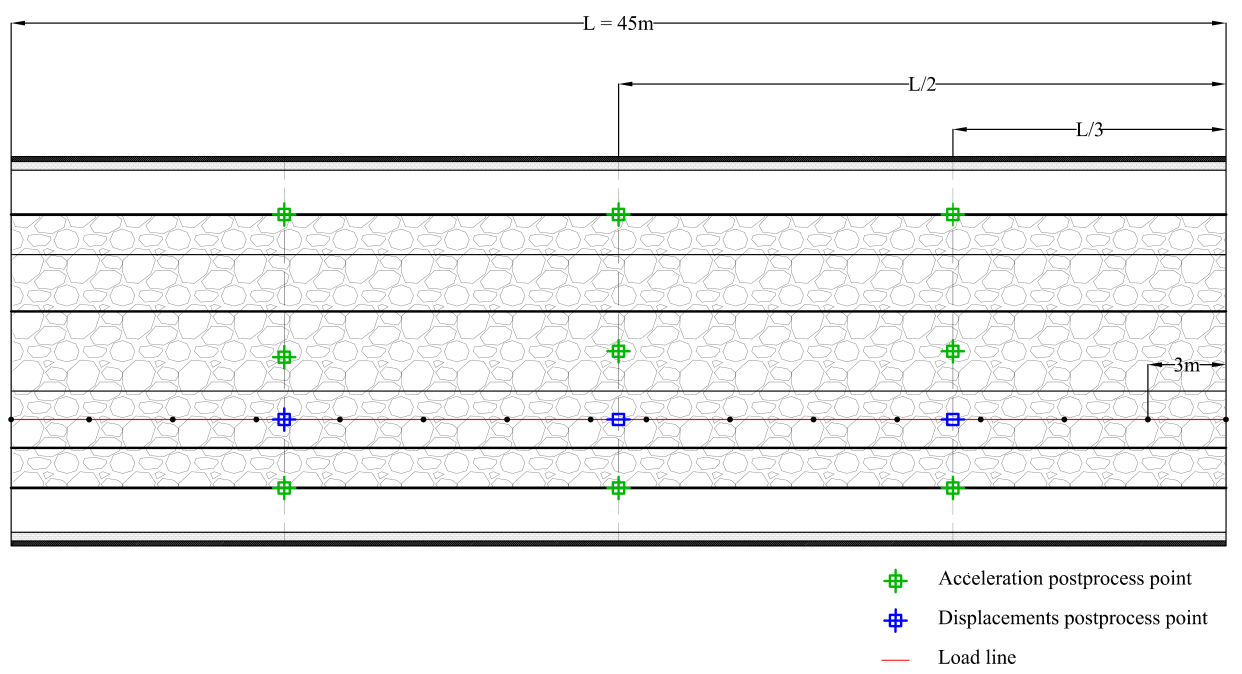

Figure 2.34: Post-process points and load line scheme.

\section{Accelerations:}

Four dynamic analysis were carried out with the conditions indicated above. The first two one were studied with a constant modal damping of $2 \%$ for all modes and the train A1, as well as the total envelope. However, it has been also developed one analysis with Rayleigh damping for which the studied train has been also the train A1 and the damping ratios have been taken from a similar structure, the Antoing Bridge. The analysis was carried out for all the post-process points, here it is only shown the results for the post-process point one, corresponding to the edge of the balast and a quarter of the length in the first span. The first two analysis try to point out the effects of the variation in the two main variables of the problem, time and velocity stepping. 
Acceleration envelope of Train A1. Effect of $\Delta v$.

For this analysis, it is determined the response of the structure in terms of acceleration under the passage of the train A1, with two different velocity steps, $10 \mathrm{~km} / \mathrm{h}$ and $20 \mathrm{~km} / \mathrm{h}$, and the same time step of $0.039 \mathrm{~s}$, corresponding to $\mathrm{T} 1 / 10$, in the proposed $\mathrm{dq} / \mathrm{dv}$ methodology. These results are compared with the Semi-Analytic solution as can be seen is figure 2.35:

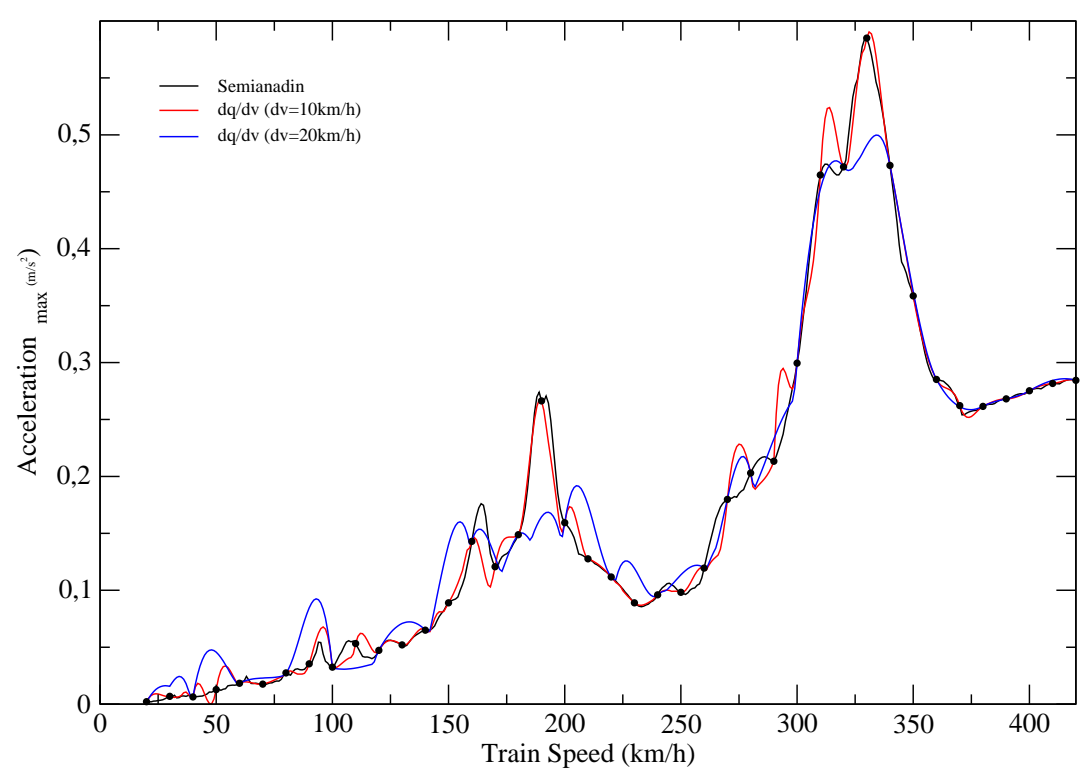

Figure 2.35: Train A1, post-process point 1. Effects of velocity stepping.

Acceleration envelope of Train A1. Effect of $\Delta t$.

In this second analysis, the scheme is the opposite, it is determined the response of the structure in terms of acceleration under the passage of the train A1, with two different time steps, 0.039 s and 0.0039 s corresponding to T1/10 and T1/100, and the same velocity step of $10 \mathrm{~km} / \mathrm{h}$ in the proposed $\mathrm{dq} / \mathrm{dv}$ methodology. Once again, these results are compared with the Semi-Analytic solution as can be seen is figure 2.36: 


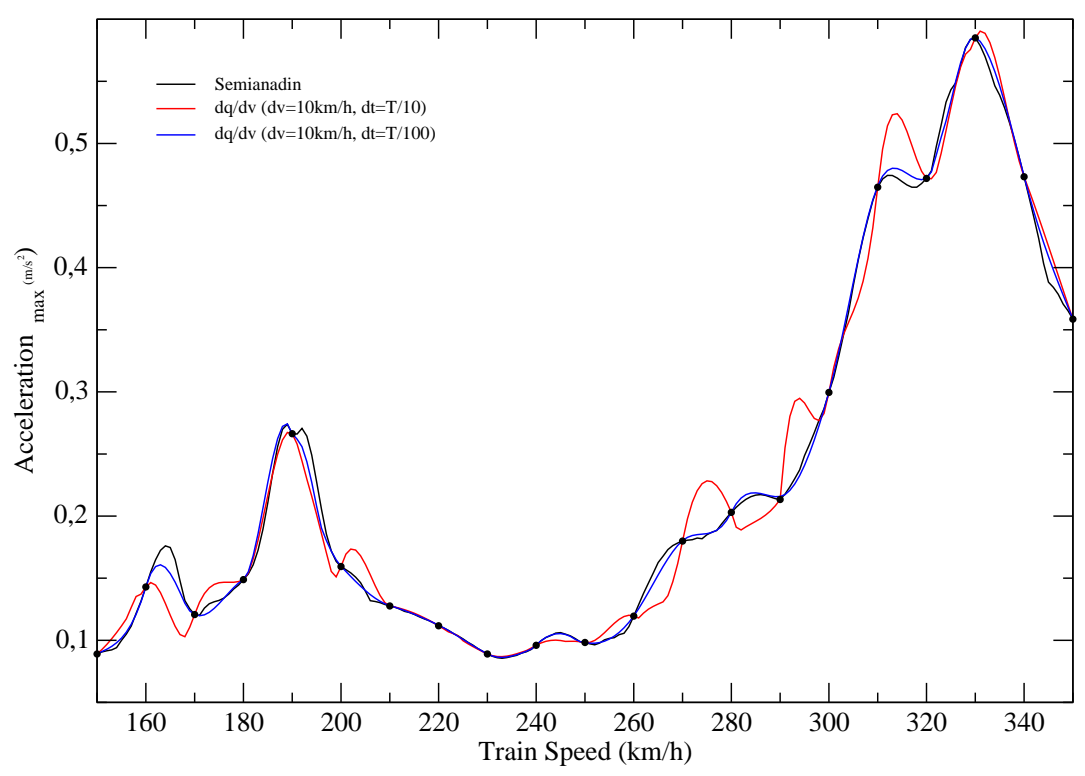

Figure 2.36: Train A1, post-process point 1. Effect of time stepping.

Acceleration envelope of Train A1. Rayleigh's damping.

In this third analysis, it is repeated the first one but with Rayleigh's damping. As indicated before, a structure of similar conditions have been taken to fix two damping ratios for two natural frequencies in the Rayleigh's damping model, Antoing Bridge, a concrete box girder bridge on the Paris-Brussels high speed railway line. The damping ratio of the mentioned structure have been taken from [16] comparing the natural modes of vibration, resulting 2.5:

\begin{tabular}{lcc} 
Mode & $\mathbf{f}(\mathbf{H z})$ & $\xi(\%)$ \\
\hline First bending mode & 2.5 & 0.63 \\
First torsional mode & 5.84 & 2.98
\end{tabular}

Table 2.5: Fixed damping ratios in Rayleigh's damping method in Rodenillo 


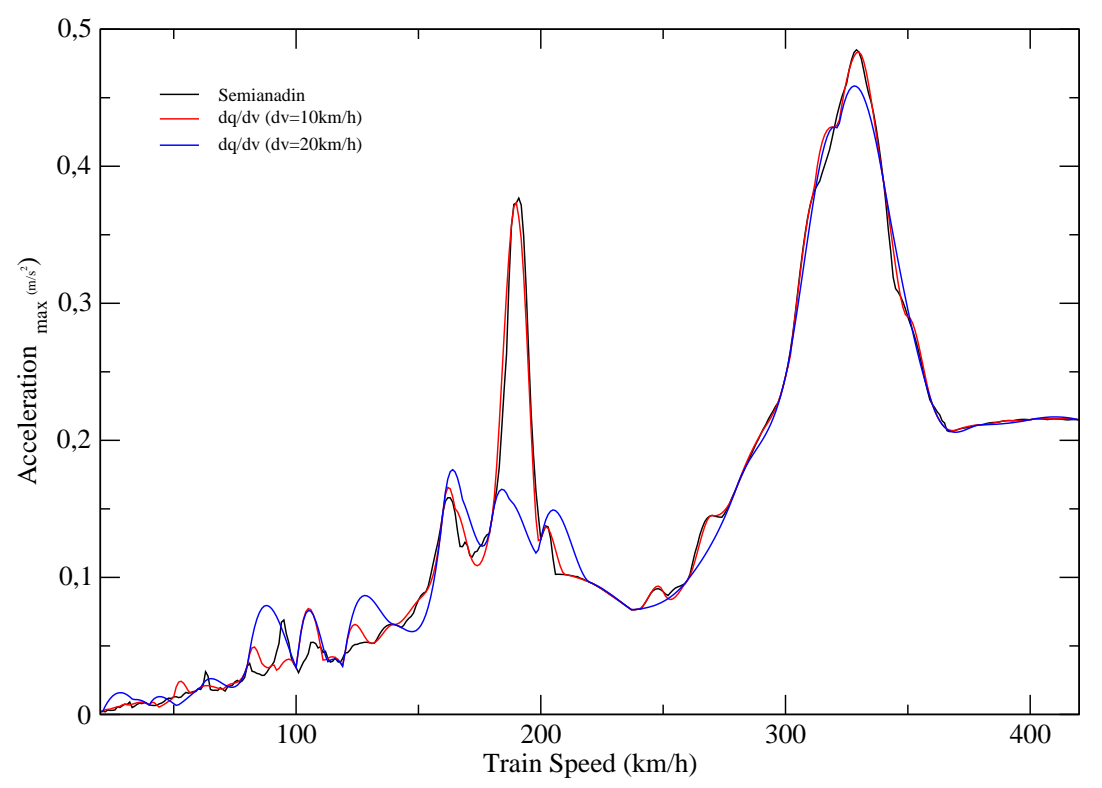

Figure 2.37: Train A1, post-process point 1. Acceleration under Rayleigh damping.

Immediately, it is noticed that the comparison with Semi-Analytic solution improves a lot. It is because the formulation of the gradient depends on the square value of the frequency, therefore, if the maximum value determination of the response is limited by the time stepping, this error is increased by the square of the frequency. Besides, what characterizes the responses of the structures with Rayleigh's damping is that the resonant response is more clean and the importance of the resonant mode is mayor in comparison with the rest of modes. This fact can be observed if a Fourier transformation is applied to two time series, one with a constant mode damping ratio and other with Rayleigh's damping. In this case, two velocities have been analyzed, 150 and $325 \mathrm{~km} / \mathrm{h}$ which correspond to a non-resonant and no-resonant situations. In the case of a non-resonant velocity, as can be seen in figures 2.38 and 2.39, the Fourier spectrum for the analysis with constant modal damping ratio is not clean and the response is due to several frequencies, in this case (fig 2.38) corresponds to the frequencies $2.3 \mathrm{~Hz}, 4.7 \mathrm{~Hz}, 4.9 \mathrm{~Hz}$ and $5.1 \mathrm{~Hz}$. However, in the case of Rayleigh damping (fig 2.39) the Fourier spectrum is more clean and the weight almost only lies on the frequency $5 \mathrm{~Hz}$ with small contributions of several frequencies else. On the other hand, the behaviors of this two models are similar as can be seen in figures 2.41 and 2.40, lying on one single frequency. This is why the proposed method approximates the solution better in the resonant velocities while the errors are higher in the non-resonant ones what makes this method interesting in pre-design phases because the maximum values (resonant velocities) are well determined with it. 

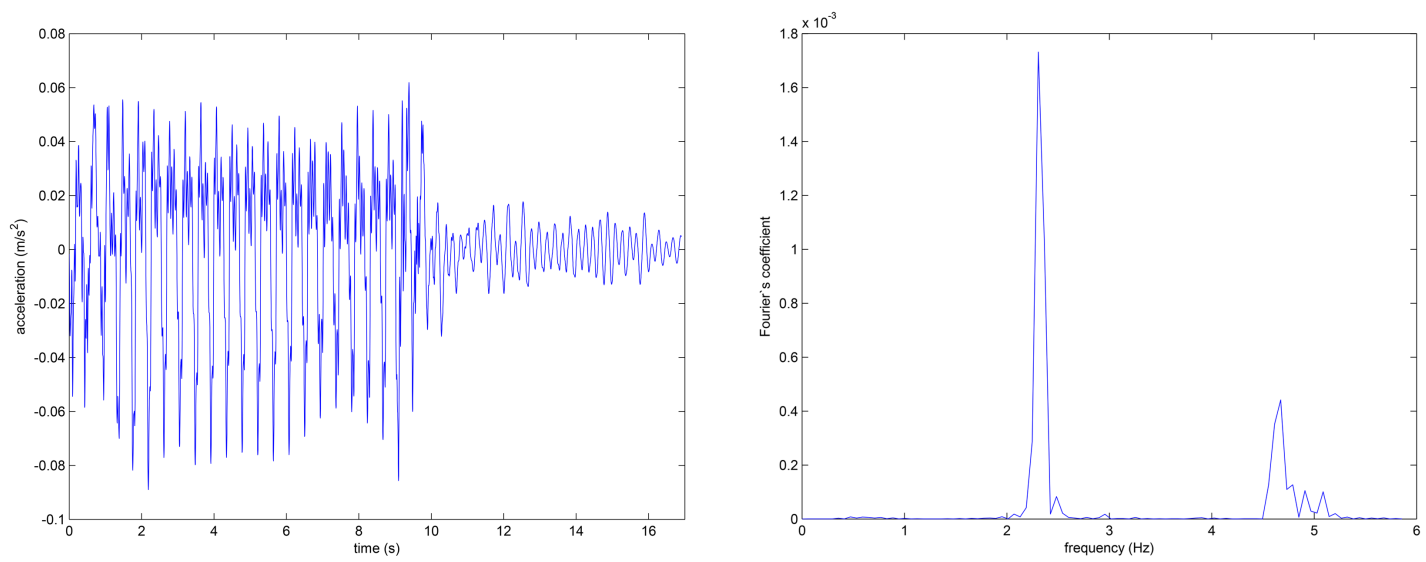

Figure 2.38: Time serie for train $\mathrm{A} 1,150 \mathrm{~km} / \mathrm{h}$ and constant modal damping ratio of $2 \%$
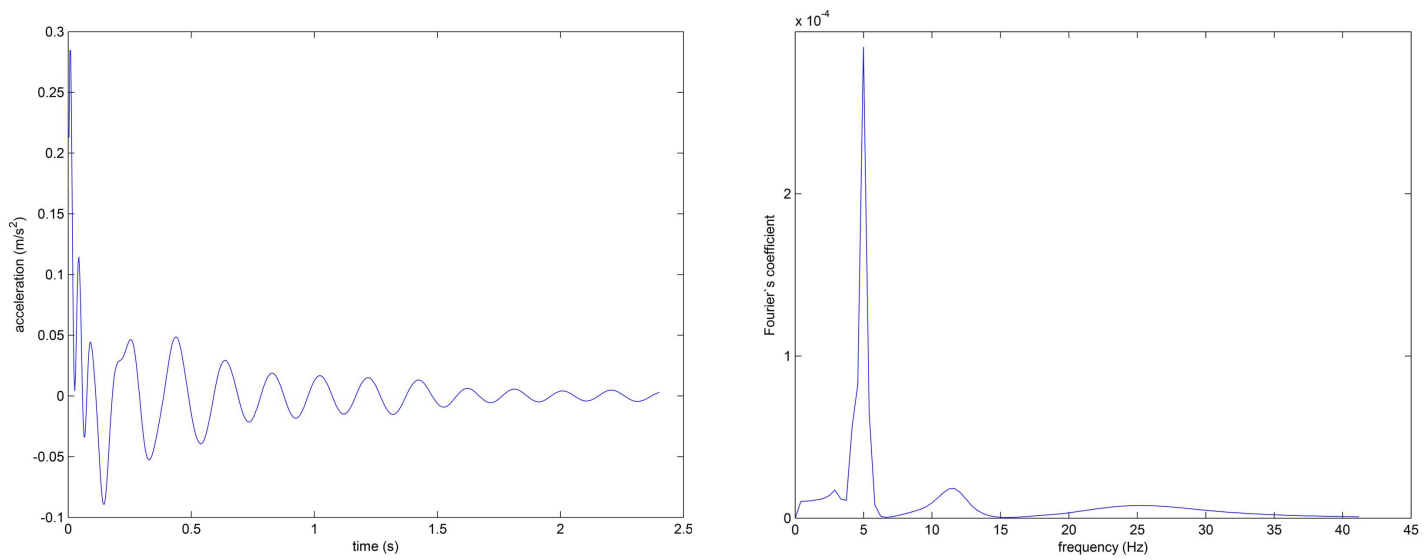

Figure 2.39: Time serie for train A1, 150km/h and Rayleigh's damping
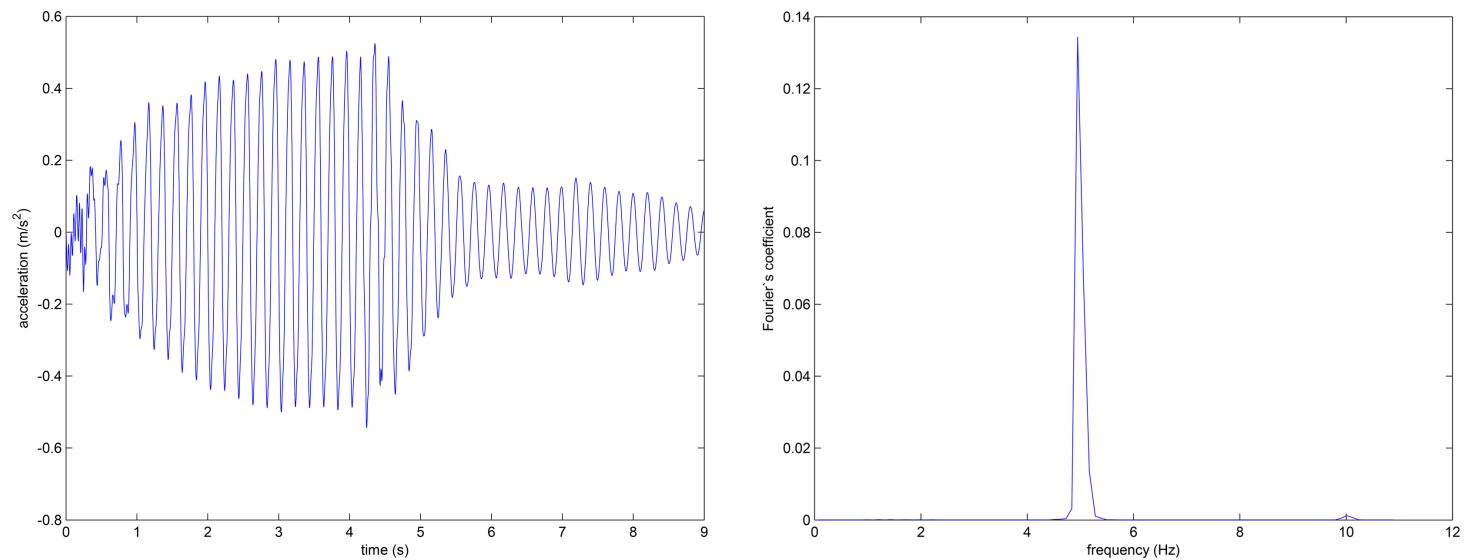

Figure 2.40: Time serie for train $\mathrm{A} 1,325 \mathrm{~km} / \mathrm{h}$ and constant modal damping ratio of $2 \%$ 

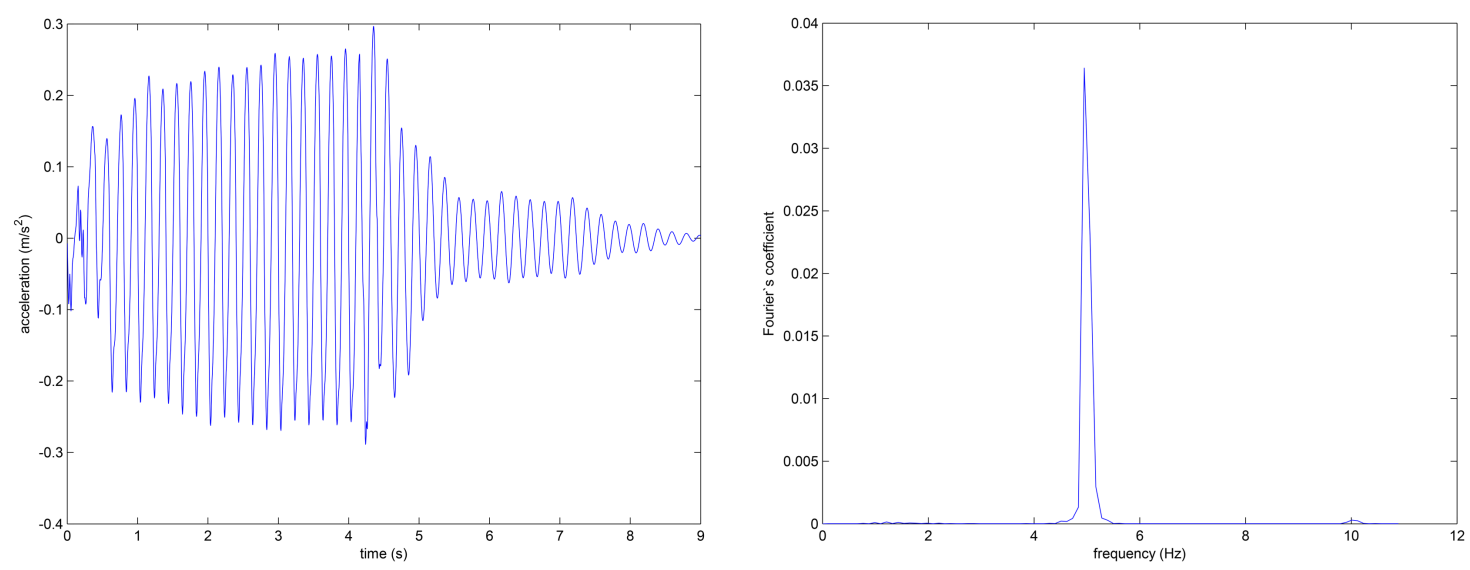

Figure 2.41: Time serie for train A1, 325km/h and Rayleigh's damping

All trains acceleration envelope.

The final objective of all this analysis is to obtain the total envelope between all the trains. In figure 2.42, it is represented the envelopes obtained by Semi-Analytic solution and the proposed method with velocity steps of $10 \mathrm{~km} / \mathrm{h}$ and $20 \mathrm{~km} / \mathrm{h}$, and all the analysis with time steps of $\mathrm{T} 1 / 10$.

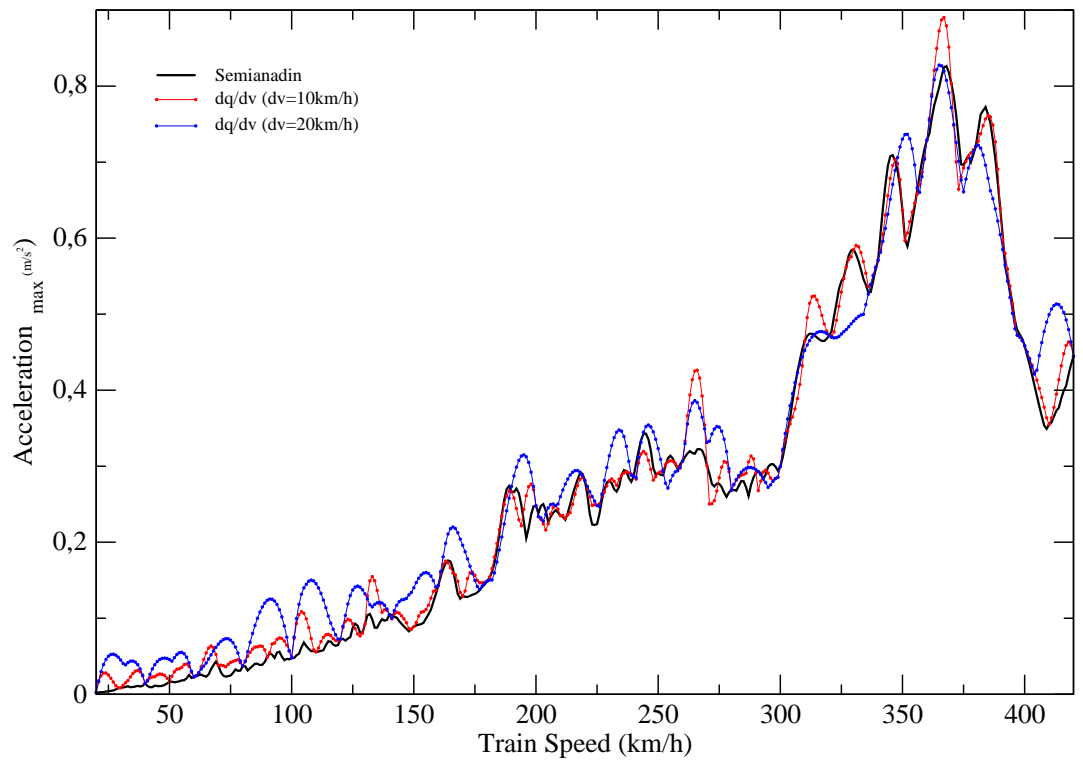

Figure 2.42: Acceleration Envelope, point 1. 
Conclusion. Relative error and time of calculus.

The benefits and the deficits of the proposed methodology can be denoted by the relative errors and the time of calculus. The relative errors are represented in figure 2.43, in which it is denoted the effects of the two main variables, time and velocity. The great reduction of the error between the envelope with time steps of $T / 10$ and $T / 100$ is because of what was indicated before, the sensitivity of the response to velocity is defined in the maximum point of the time series so an error in its determination shall be amplified by the square of the frequency. Anyway, the advantage of this method is that the errors lays under $20 \%$ in the resonant-velocities $(350-400 \mathrm{~km} / \mathrm{h})$ where the accelerations are higher.

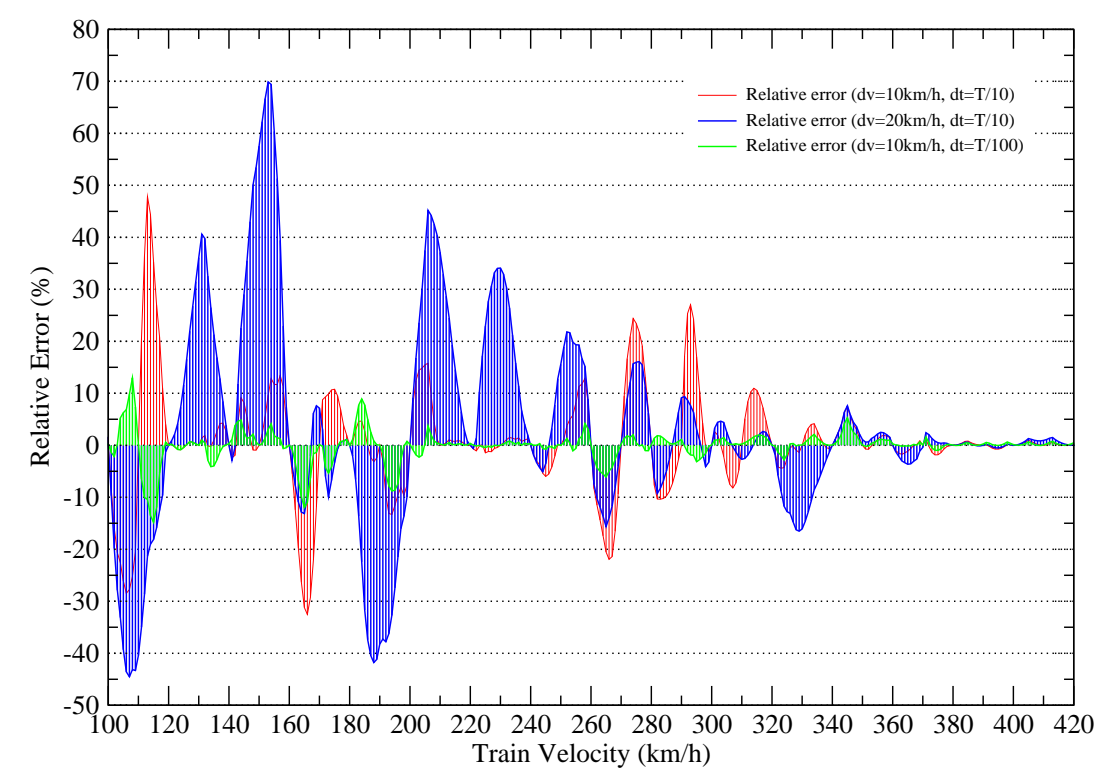

Figure 2.43: Relative error on acceleration envelopes of point 1.

In terms of time of calculus, the time required to obtain the curves of the figure 2.43 are:

- Semianalytic solution $\left(\Delta \mathrm{v}=1 \mathrm{~km} / \mathrm{h}, \Delta \mathrm{t}=T_{1} / 10\right)=10518.56 \mathrm{~s}$

- $\mathrm{dq} / \mathrm{dv}$ solution $\left(\Delta \mathrm{v}=10 \mathrm{~km} / \mathrm{h}, \Delta \mathrm{t}=\mathrm{T}_{1} / 10\right)=1120.78 \mathrm{~s}$ (reduction of $89 \%$ )

- $\mathrm{dq} / \mathrm{dv}$ solution $\left(\Delta \mathrm{v}=10 \mathrm{~km} / \mathrm{h}, \Delta \mathrm{t}=\mathrm{T}_{1} / 100\right)=9969.78 \mathrm{~s}$ (reduction of $5 \%$ )

- $\mathrm{dq} / \mathrm{dv}$ solution $\left(\Delta \mathrm{v}=20 \mathrm{~km} / \mathrm{h}, \Delta \mathrm{t}=\mathrm{T}_{1} / 10\right)=608.53 \mathrm{~s}$ (reduction of $94 \%$ )

The study of this two magnitudes defines the utility of this proposed method as an approximated analysis in the study of different alternatives during the design of a railway bridge. With this, depending on the accuracy that the designer needs in relation with the phase of design that he is facing, he will be able to analyze different alternatives without spending long time of calculus in each case. 


\section{Displacements:}

For displacements, one again, the same scheme of four dynamic analysis were developed. As for accelerations, the analysis was done for all the post-process points, however, here it is only shown the results for the post-process point one, corresponding to the center of the deck and a quarter of the length in the first span.

\section{Displacement envelope of Train A1. Effect of $\Delta v$.}

It was determined the response of the structure in terms of displacements under the passage of the train A1, with two different velocity steps, $10 \mathrm{~km} / \mathrm{h}$ and $20 \mathrm{~km} / \mathrm{h}$, and the same time step of $0.039 \mathrm{~s}$, corresponding to $\mathrm{T} 1 / 10$, in the proposed $\mathrm{dq} / \mathrm{dv}$ methodology. These results are compared with the Semi-Analytic solution as can be seen is figure 2.44:

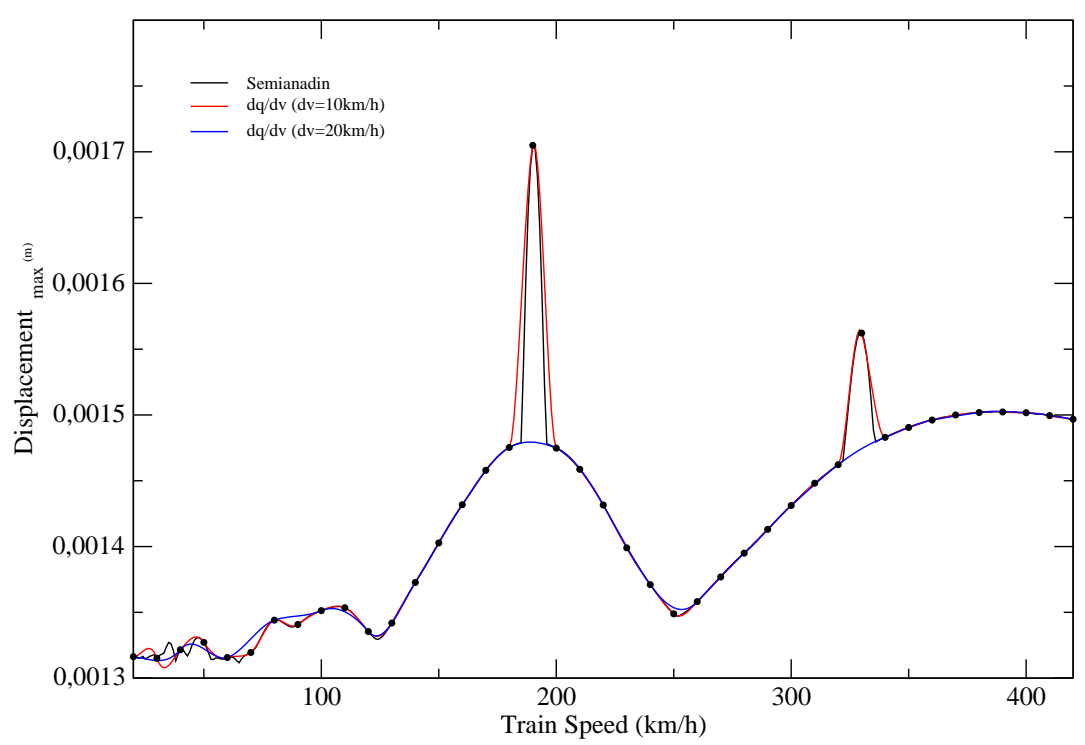

Figure 2.44: Train A1, post-process point 1. Effect of velocity stepping.

Displacement envelope of Train A1. Effect of $\Delta t$.

In this second analysis, it was determined the response of the structure in terms of displacements under the passage of the train A1, with two different time steps, $0.039 \mathrm{~s}$ and $0.0039 \mathrm{~s}$ corresponding to $\mathrm{T} 1 / 10$ and $\mathrm{T} 1 / 100$, and the same velocity step of $10 \mathrm{~km} / \mathrm{h}$ in the proposed $\mathrm{dq} / \mathrm{dv}$ methodology. The results were compared with the Semi-Analytic solution as can be seen is figure 2.45 : 


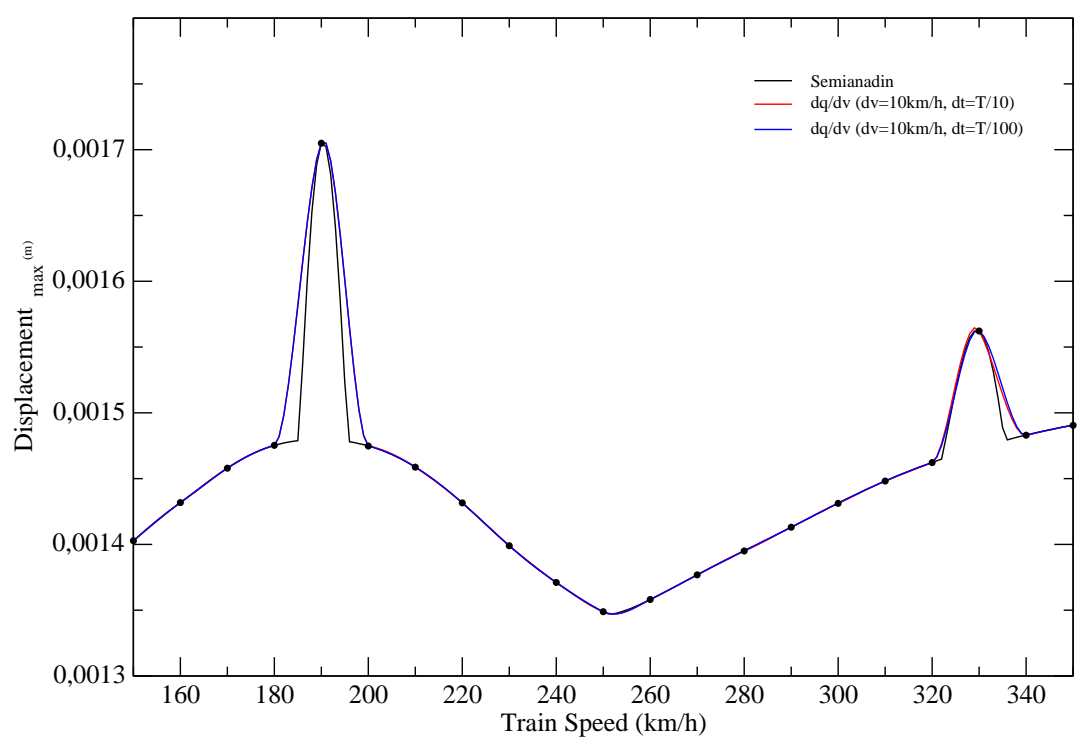

Figure 2.45: Train A1, post-process 1. Effect of time stepping.

Displacement envelope of Train A1. Rayleigh's damping.

Here it is also carried out the analysis with Rayleigh's damping. As indicated above, the damping ratios of the bridge have been taken comparing with the measurements in Antoing Bridge ([16]), resulting 2.46:

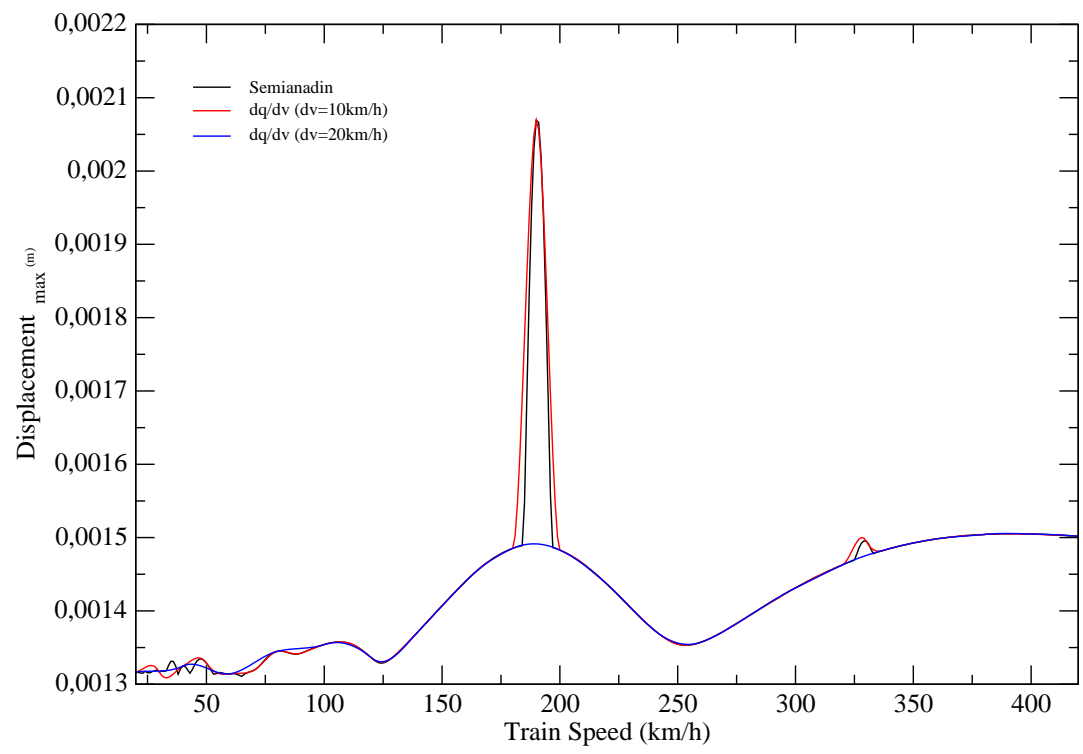

Figure 2.46: Train A1, post-process 1. Displacement evaluation under Rayleigh damping.

For displacements the improvements in the approximation with the proposed method are not so remarkable. This is due to the formulation in which, for displacement, the results 
are not proportional to frequency, that is why the approximation are already good enough in the first analysis.

\section{All train displacement envelope.}

In figure 2.47, it is represented the envelopes obtained by Semi-Analytic solution and the proposed method with velocity steps of $10 \mathrm{~km} / \mathrm{h}$ and $20 \mathrm{~km} / \mathrm{h}$, and all the analysis with time steps of $\mathrm{T} 1 / 10$.

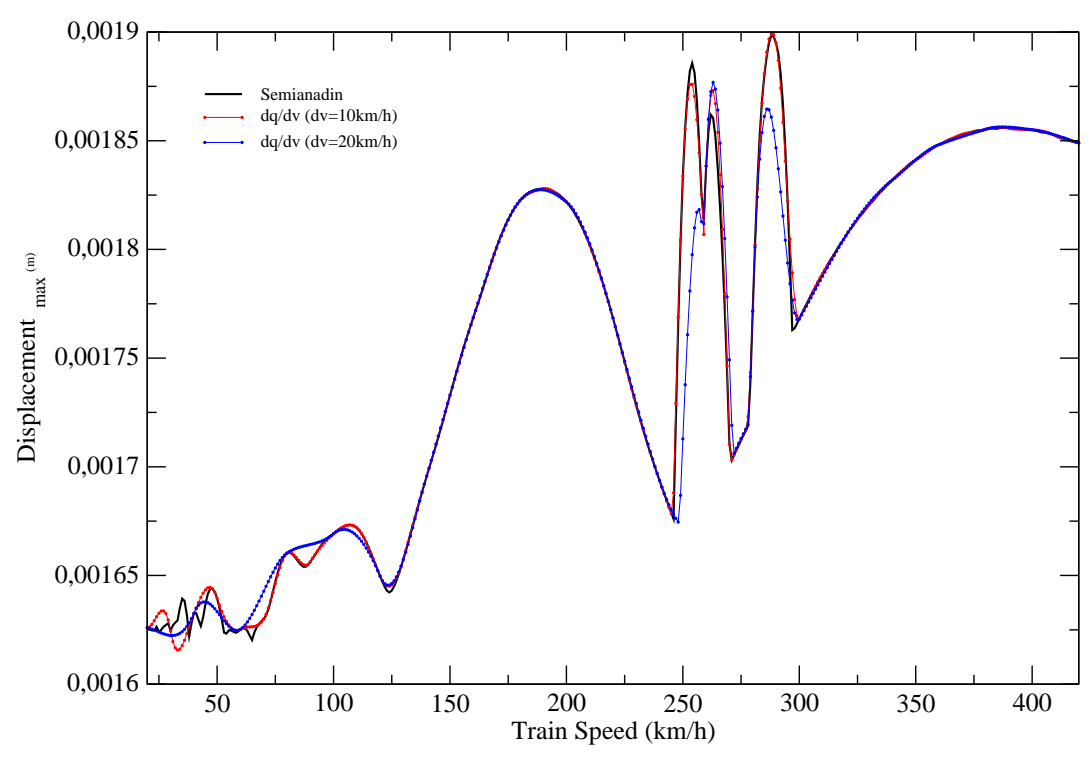

Figure 2.47: Displacement envelope on post-process point 1.

Conclusion. Relative error and time of calculus.

The relative errors are represented in figure 2.48. Here, all the errors lay under $14 \%$ and the reason is the same as indicated in the Rayleigh's damping analysis: the displacement are not direct proportional to frequency, that is why the approximation is not necessary better around the resonant velocities. Besides, the displacement series are not so fluctuating as the acceleration ones, this allows to obtain the maximum values with enough accuracy with bigger time steps. 


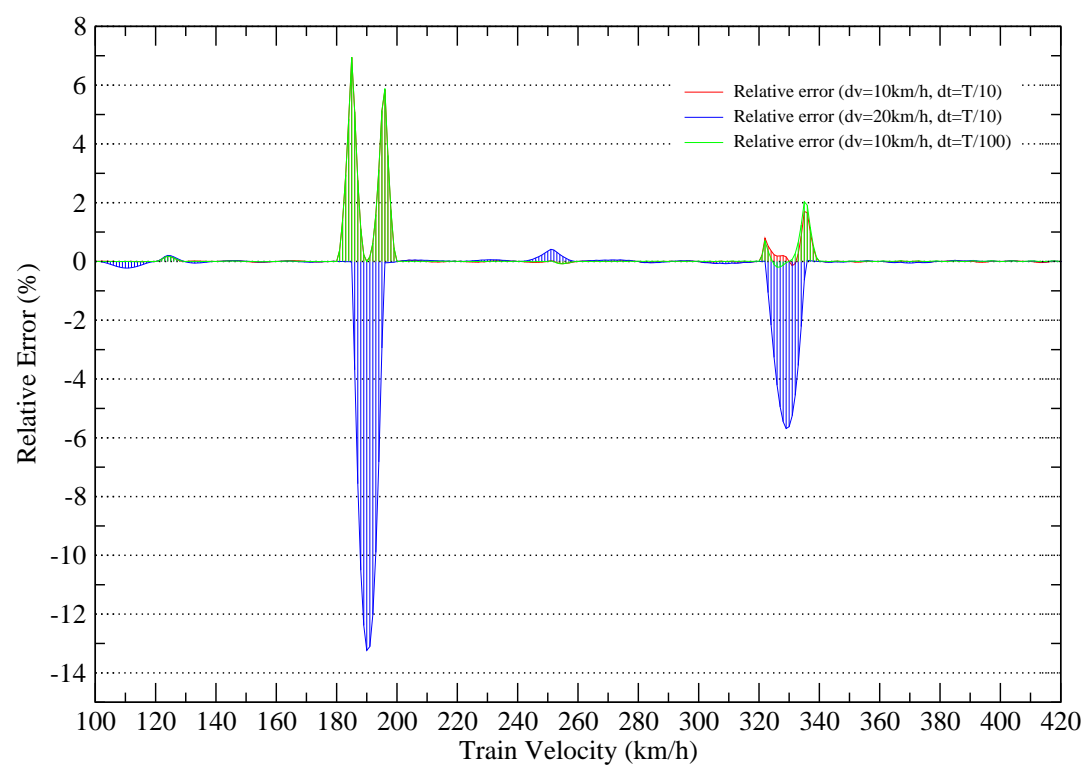

Figure 2.48: Relative error on displacement envelopes of point 1.

The analysis are developed together for displacements and acceleration so the time of calculus are the same as the ones indicated before. Nevertheless, here the reductions of time are obviously much more important.

\subsubsection{Steel-concrete composite bridge. Sesia viaduct.}

In this test, a steel-concrete composite high-speed bridge is analyzed for which a real case is chosen, the Sesia Viaduct, a widely studied bridge in the literature. A finite element model is developed and validated by the wide information in the literature, not only numerically but experimentally. This bridge is defined by a steel box girder sustaining a concrete deck with two lines resulting an interesting case in order to determine the influence of the torsional eefects in the proposed methodology.

\section{Description of the Sesia viaduct}

The Sesia viaduct is situated on the Italian high speed railway line between Torino and Milano. It is a steel-concrete composite bridge composed of seven $46 \mathrm{~m}$ long simply supported, resulting in a total length of $322 \mathrm{~m}$. The steel box girder is divided into two segments $\mathrm{C} 1$ and $\mathrm{C} 2$, which are connected by full penetration butt welding. As it is defined in figure 2.49, there are fifteen cross diaphragms at the interval of $3.114 \mathrm{~m}$ which support the necessary lateral stiffness to limit the distortion of the steel box girder. Two characteristic cross section are defined in each span as can be seen in figure 2.50 , one at the bridge bearing (DS) and the other along the rest of the structure (D1). The twin-box steel girder, with an overall width of $10.2 \mathrm{~m}$ and a depth of $3.35 \mathrm{~m}$, is covered by a $13.6 \mathrm{~m}$ wide and $0.4 \mathrm{~m}$ thick concrete deck, which 
is connected to the top flanges of the steel box girder by shear studs. Ballasted double tracks with UIC60 rails are supported at every $0.6 \mathrm{~m}$ distance by prestessed concrete sleepers.

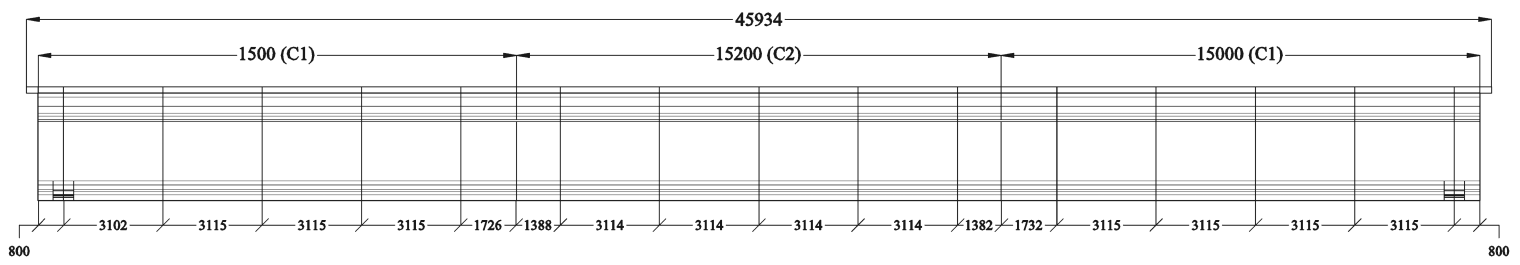

a) Elevation view

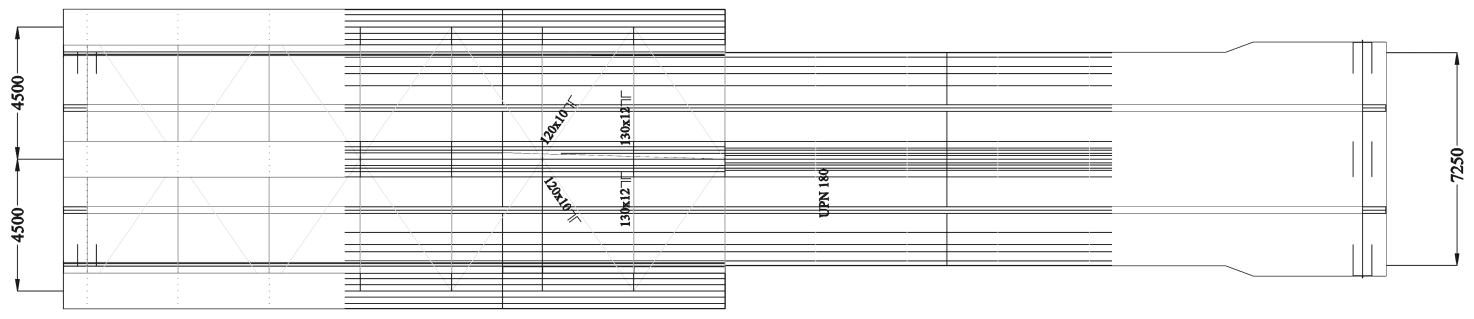

b) Plan view

Figure 2.49: Elevation and front view of Sesia.
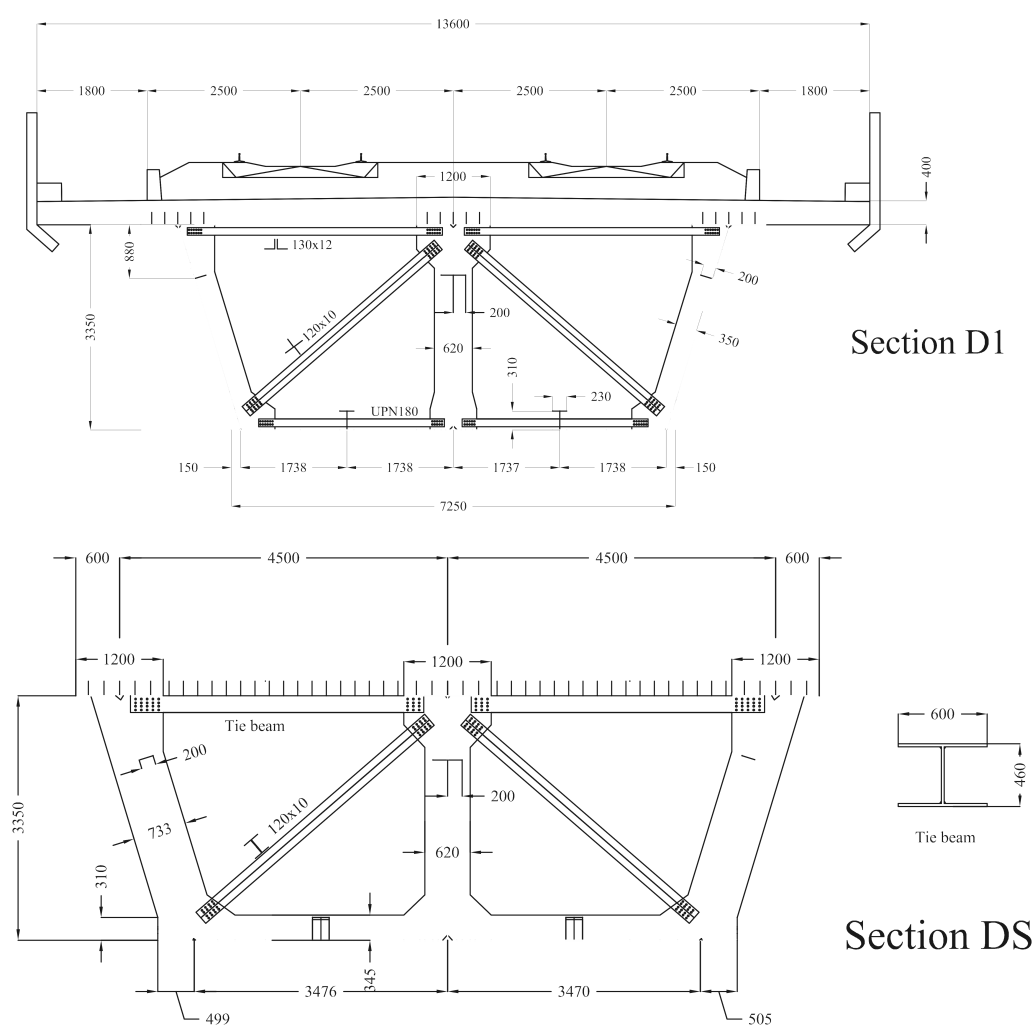

Figure 2.50: Cross sections of viaduct of Sesia. 


\section{FEM model}

Due to the large size of the Sesia viaduct including every simply supported spans, the FEM model of the overall bridge would result in a large computational cost. Therefore, a simplified model of a single span was modeled with appropriate boundary conditions on the rails to simulate the continuity of the bridge. Taking advantage of the symmetry and the similarity of the bridge, the span was divided into three types of segments. The three types of segments were separately created and meshed into details. The segments B1 and B2 are identical in profile except for the plate thickness in webs and bottom flanges, while the values of the thickness in segment B1 for webs and bottom flanges are $20 \mathrm{~mm}$ and $25 \mathrm{~mm}$ respectively, in segment B2 they measures $18 \mathrm{~mm}$ and $30 \mathrm{~mm}$. For the whole structure, the top flanges are $25 \mathrm{~mm}$. The diagonal and horizontal bracings were modeled by beam elements, and their cross sections are defined in figure 2.49. Besides, the stiffeners are also modeled with beam elements defining equivalent flanges and defining reduction factors for duplicated masses. The steel box girder was simulated by shell element, as well as the concrete deck which is connected to the top flanges of the box and the horizontal bracings of the diaphragms at the bearings through shear studs, modeled as massless infinite rigid beams. In order to simulate the effect of the increased reinforcement in the concrete deck through its transversal direction, the beam rigidity of the concrete deck is increased by $20 \%$, a good approximation in experience. The final model is defined by 3280 frame elements, 12304 shell elements and 13152 joints. In reference to the boundaries, symmetrical boundary conditions were adopted at the ends of the span as shown in fig 2.51. Furthermore, the longitudinal direction in the ends of the rails are fixed in order to simulate the connection with the adjacent spans, besides, the vertical displacement and the transverse rotation are limited in the center of the rails in order to obtain a smooth load line to the following dynamic analysis and reducing the impact effect at the entrance of the trains. The masses and the properties of the employed materials are summarized in the table 2.6. Notice the density of steel is elevated to $8000 \mathrm{~kg} / \mathrm{m}^{3}$ in order to take into account the masses of welds, bolts, ancillaries and all the auxiliary elements that have not been defined in the model. 


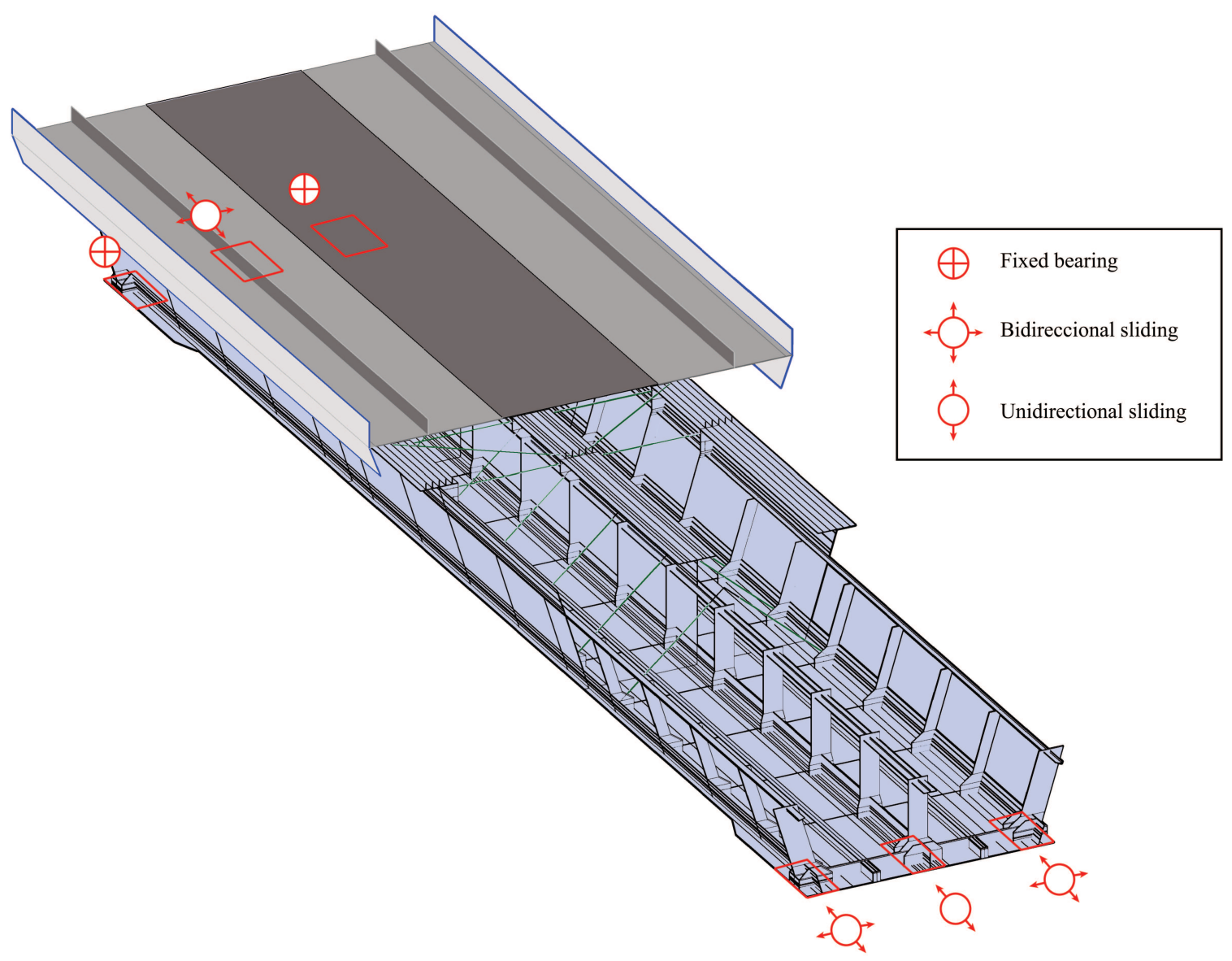

Figure 2.51: Boundary conditions on Sesia viaduct.

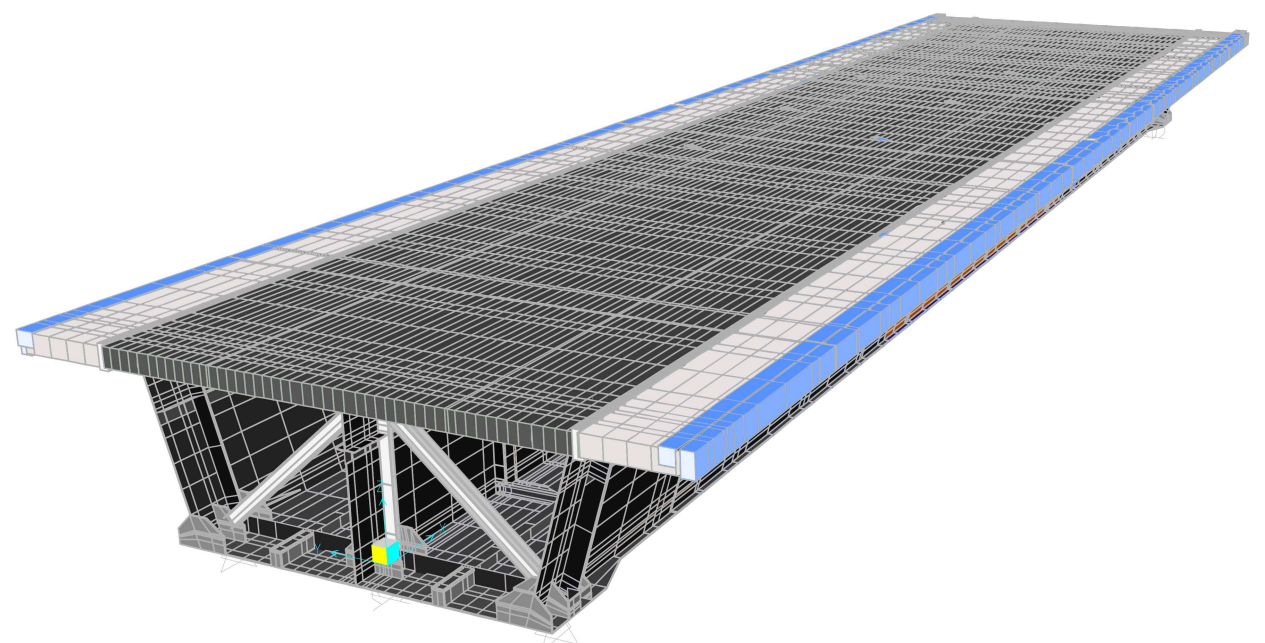

Figure 2.52: 3D FEM model of Viaduct sesia. 


\begin{tabular}{lcc}
\hline Item & Unit & Value \\
\hline Per-unit-length mass of rail & $\mathrm{kgf} / \mathrm{m}$ & 60 \\
Mass of sleepers & $\mathrm{kg}$ & 290 \\
Young's modulus of concrete slab (C3D8) & $\mathrm{Mpa}$ & 31000 \\
Poisson's ration of concrete slab (C3D8) & - & 0.17 \\
Density of concrete slab (C3D8) & $\mathrm{kg} / \mathrm{m}^{3}$ & 2500 \\
Young's modulus of steel box (S4) & $\mathrm{Mpa}$ & 205000 \\
Poisson's ration of steel box (S4) & - & 0.3 \\
Density of steel box (S4) & $\mathrm{kg} / \mathrm{m}^{3}$ & 8000 \\
\hline
\end{tabular}

Table 2.6: Main materiales in the Sesia's FEM model.

\section{Modal Analysis}

The modal analysis was computed using the FEm model described in the previous section using the software SAP2000. As the Spanish Standard IAPF indicates, the total number of modes taken into consideration are all those whose natural frequency have a value below $30 \mathrm{~Hz}$, limit value that the experience indicates that deconsolidates the ballast, with that, the analysis takes into consideration a total of 82 modes. The values have been compared with studies in the literature as (1)[46], (2) [47] and (3) [48], reaching acceptable values:

\begin{tabular}{|c|c|c|c|c|c|}
\hline \multirow{2}{*}{ Vibration mode } & \multicolumn{5}{|c|}{ Natural frequencies $[\mathrm{Hz}]$} \\
\hline & \multicolumn{2}{|c|}{ [1] } & {$[2]$} & {$[3]$} & Current study \\
\hline The first bending mode & 4.14 & 4.15 & 4.2 & 4.15 & 4.19 \\
\hline The first torsional mode & 9 & 9.22 & 9.3 & 9.01 & 9.68 \\
\hline The second bending mode & 10.44 & 10.51 & 11.69 & 10.27 & 10.83 \\
\hline
\end{tabular}

Table 2.7: Natural frequencies of Sesia viaduct.

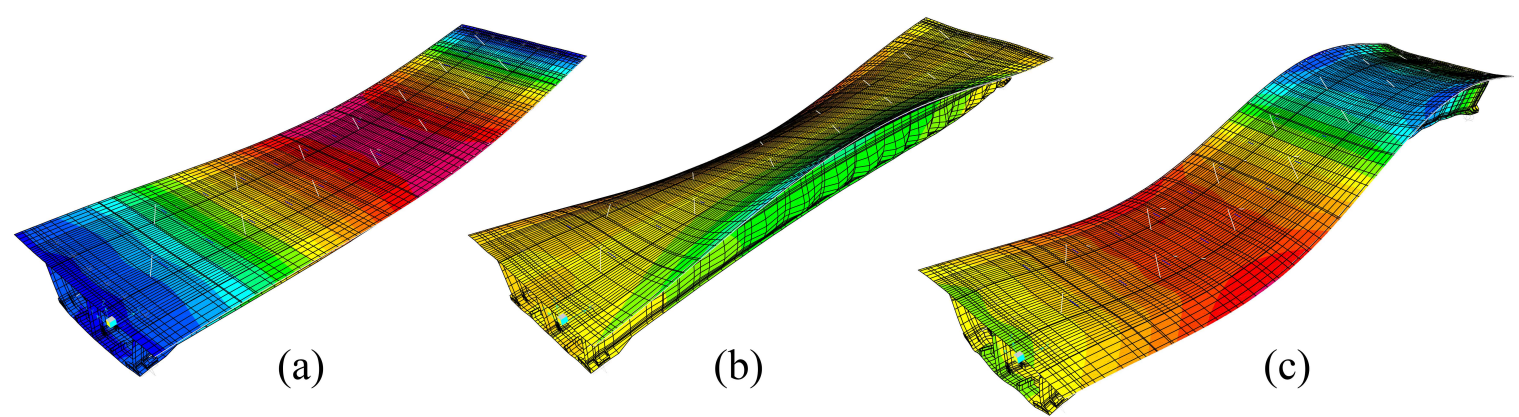

Figure 2.53: Natural modes of the Sesia viaduct. 


\section{Dynamic analysis}

\section{Accelerations:}

The scheme of the analysis was exact the same as the one before. Four dynamic analysis were carried out, the first two one with a constant modal damping of $2 \%$ for all modes and the train A1, the total envelope, and an analysis with Rayleigh damping. In this case, the damping ratios have been taken from real measurements in this bridge, [48], [46], [47] and [49]. The analysis was carried out for all the post-process points, here it is only shown the results for the post-process point five in acceleration, corresponding to the center of the ballast and half length of the span, and point 2 in displacement, corresponding to the center of the line and half length of the span.

\section{Acceleration envelope of train A1. Effect of $\Delta v$.}

As the latter test, it was determined the response of the structure in terms of acceleration under the passage of the train A1, with two different velocity steps, $10 \mathrm{~km} / \mathrm{h}$ and $20 \mathrm{~km} / \mathrm{h}$, and the same time step of $0.039 \mathrm{~s}$, corresponding to $\mathrm{T} 1 / 10$, in the proposed $\mathrm{dq} / \mathrm{dv}$ methodology. These results are compared with the Semi-Analytic solution as can be seen is figure 2.54:

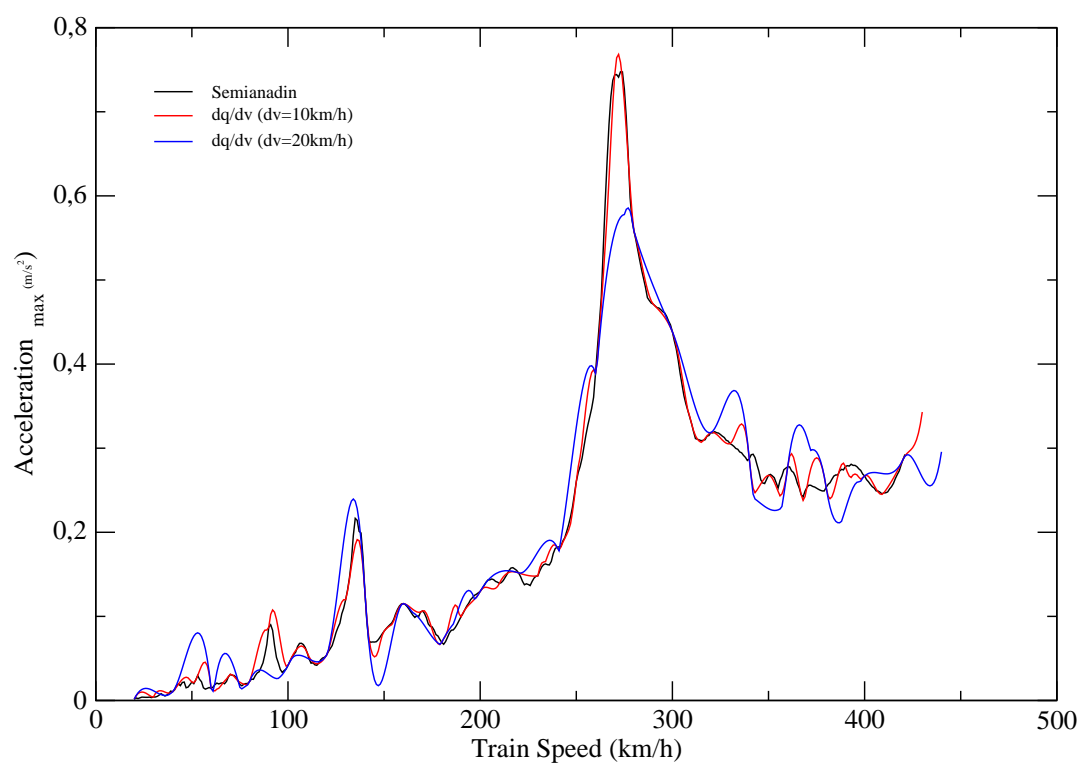

Figure 2.54: Train A1, post-process point 5. Effects of velocity stepping.

Acceleration envelope of train A1. Effect of $\Delta t$.

In this second analysis, it is determined the response of the structure in terms of acceleration under the passage of the train A1, with two different time steps, $0.039 \mathrm{~s}$ and $0.0039 \mathrm{~s}$ corresponding to $\mathrm{T} 1 / 10$ and $\mathrm{T} 1 / 100$, and the same velocity step of $10 \mathrm{~km} / \mathrm{h}$ in the proposed 
$\mathrm{dq} / \mathrm{dv}$ methodology. These results were compared with the Semi-Analytic solution as can be seen is figure 2.55 :

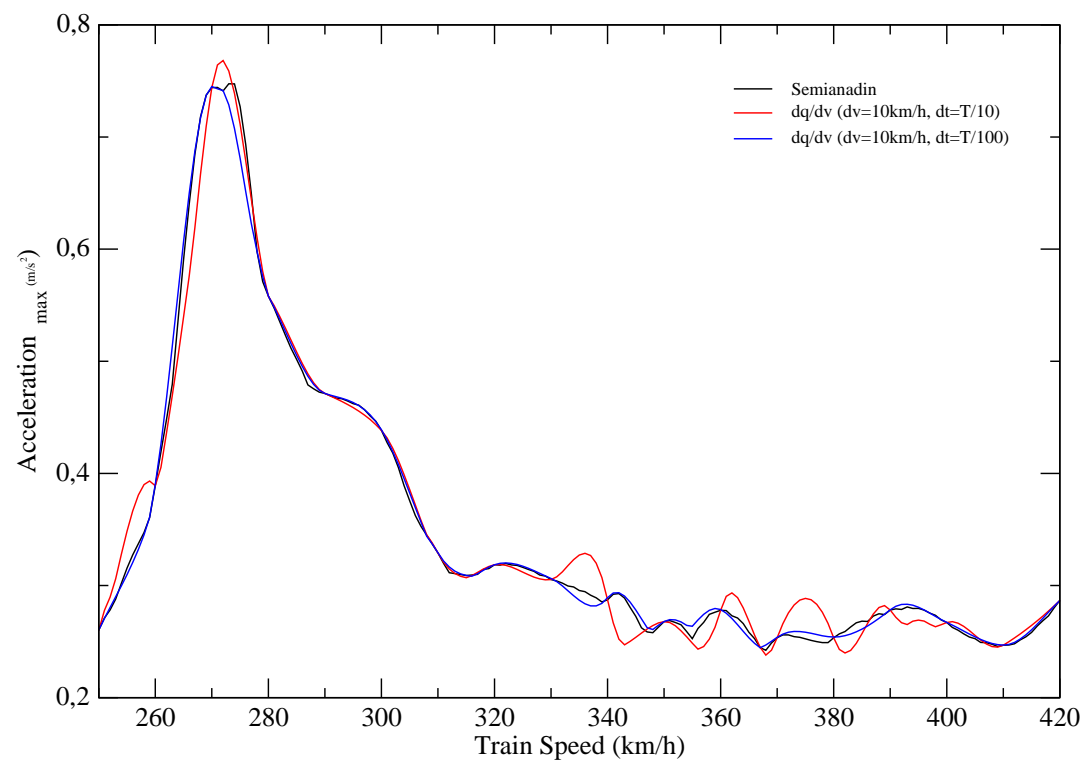

Figure 2.55: Train A1, post-process point 5. Effects of time stepping.

Acceleration envelope of train A1. Rayleigh's damping.

It was repeated the first test but with Rayleigh's damping. As indicated before, there are several studies in the literature, [48],

\begin{tabular}{lcc} 
Mode & $\mathbf{f}(\mathbf{H z})$ & $\boldsymbol{\xi}(\mathbf{\%})$ \\
\hline First bending mode & 4.14 & 2.17 \\
First torsional mode & 9 & 1.84
\end{tabular}

Table 2.8: Fixed damping ratios in Rayleigh's damping method in Sesia viaduct. 


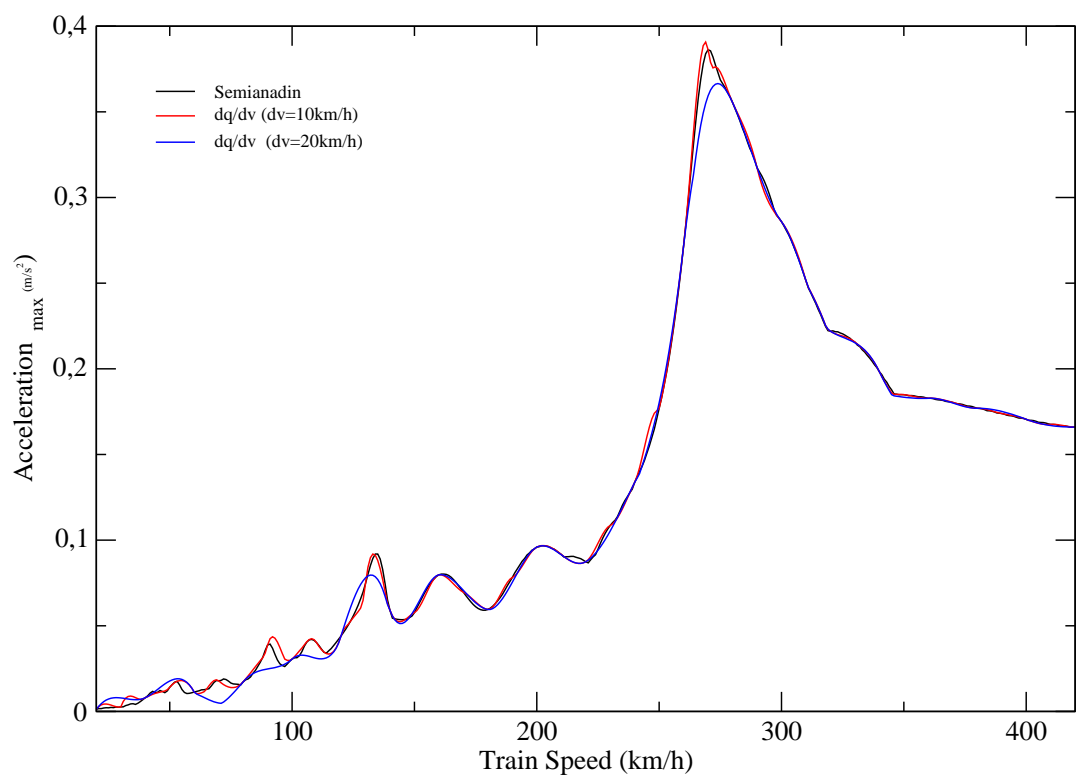

Figure 2.56: Train A1, post-process point 5. Acceleration evaluation under Rayleigh damping.

The observation of the result throws the same conclusions: response with Rayleigh's damping is better approximated with $\mathrm{dq} / \mathrm{dv}$ method than the constant modal damping model. The explanation is the same, it is because the formulation of the gradient depends on the square value of the frequency, thus, if the maximum value determination of the response is limited by the time stepping, the errors are increased by the square of the frequency. Besides, as it could be checked in the last test, what characterizes the responses of the structures with Rayleigh's damping is that the resonant response is more clean and the importance of the resonant mode is mayor in comparison with the rest of modes.

All trains acceleration envelope.

It was determined the total envelope between all the trains. In figure 2.57 , it is represented the envelopes obtained by Semi-Analytic solution and the proposed method with velocity steps of $10 \mathrm{~km} / \mathrm{h}$ and $20 \mathrm{~km} / \mathrm{h}$, and all the analysis with time steps of T1/10. 


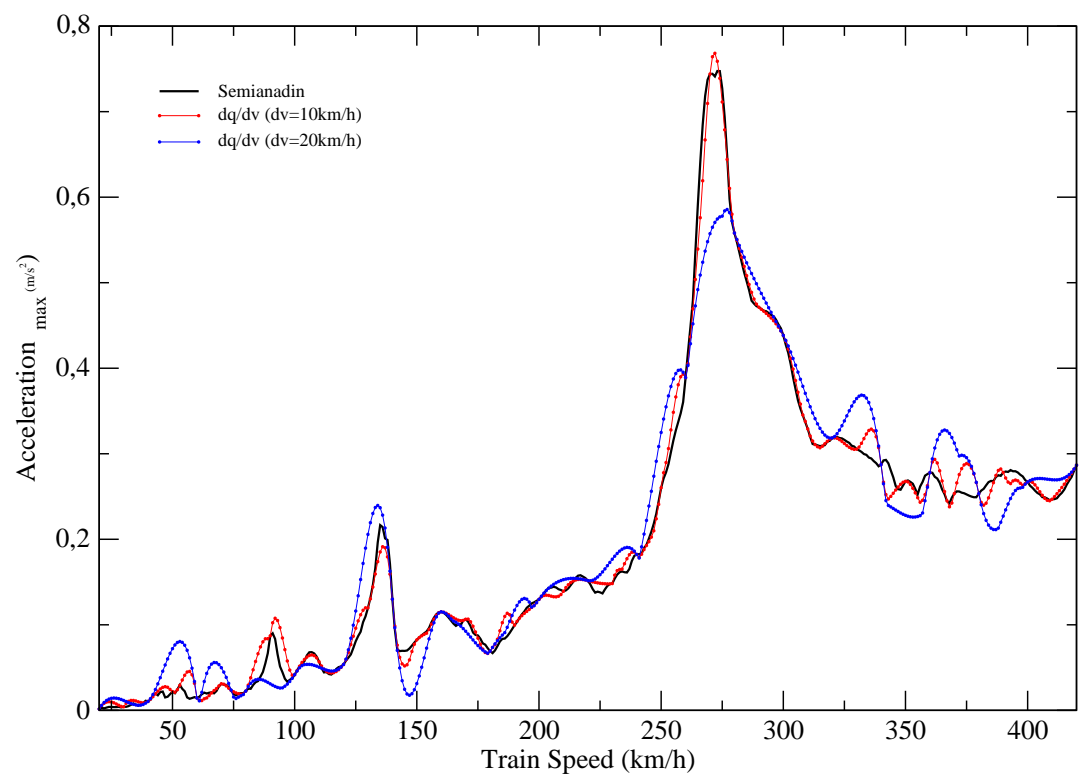

Figure 2.57: Acceleration envelope on post-process point 5 .

Conclusion. Relative errors and time of calculus.

The relative errors are represented in figure 2.58. In this case, the errors can reach elevate values for high velocity steps.

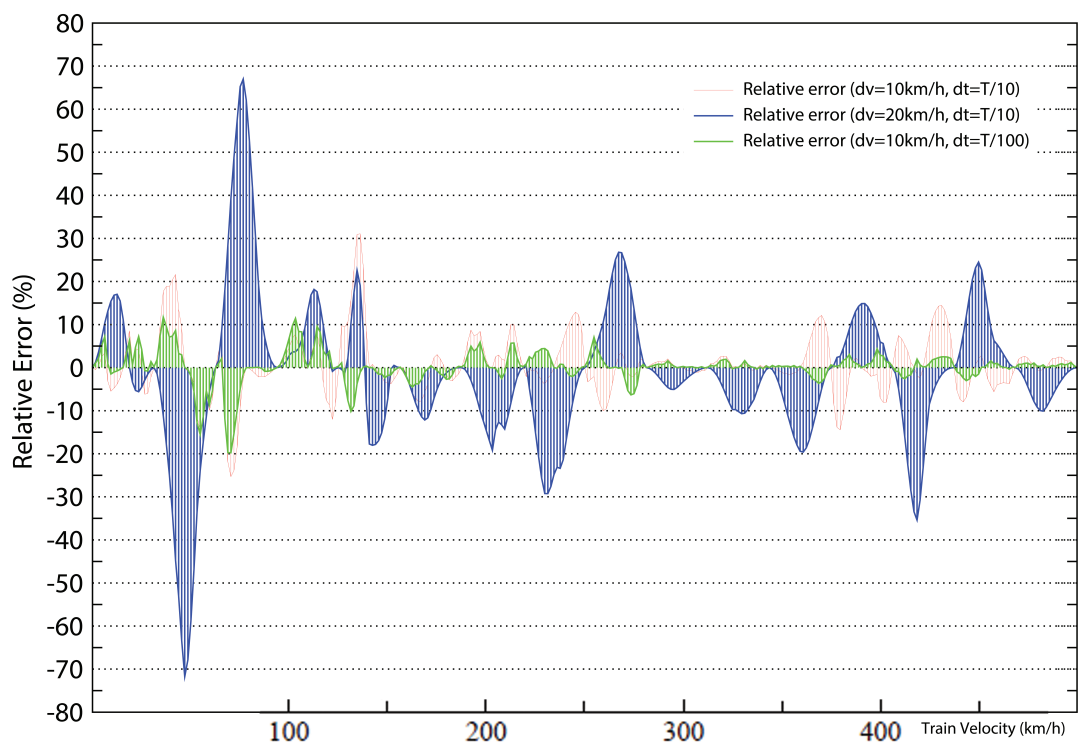

Figure 2.58: Relative error on acceleration envelopes of point 5.

In terms of time of calculus, the time required to obtain the curves of the figure 2.43 are:

- Semianalitic solution $\left(\Delta \mathrm{v}=1 \mathrm{~km} / \mathrm{h}, \Delta \mathrm{t}=T_{1} / 10\right)=1388.29 \mathrm{~s}$

- $\mathrm{dq} / \mathrm{dv}$ solution $\left(\Delta \mathrm{v}=10 \mathrm{~km} / \mathrm{h}, \Delta \mathrm{t}=\mathrm{T}_{1} / 10\right)=163.63 \mathrm{~s}$ (reduction of $11.80 \%$ ) 
- $\mathrm{dq} / \mathrm{dv}$ solution $\left(\Delta \mathrm{v}=10 \mathrm{~km} / \mathrm{h}, \Delta \mathrm{t}=T_{1} / 100\right)=1517.50 \mathrm{~s}$ (reduction of $109.31 \%$ )

- $\mathrm{dq} / \mathrm{dv}$ solution $\left(\Delta \mathrm{v}=20 \mathrm{~km} / \mathrm{h}, \Delta \mathrm{t}=\mathrm{T}_{1} / 10\right)=89.67 \mathrm{~s}$ (reduction of $6.46 \%$ )

The study of this two magnitudes defines the utility of this proposed method as an approximated analysis in the study of different alternatives during the design of a railway bridge. With this, depending on the accuracy that the designer needs in relation with the phase of design that he is facing, he will be able to analyze different alternatives without spending long time of calculus in each case.

\section{Displacements:}

Displacement envelope of train A1. Effect of $\Delta v$.

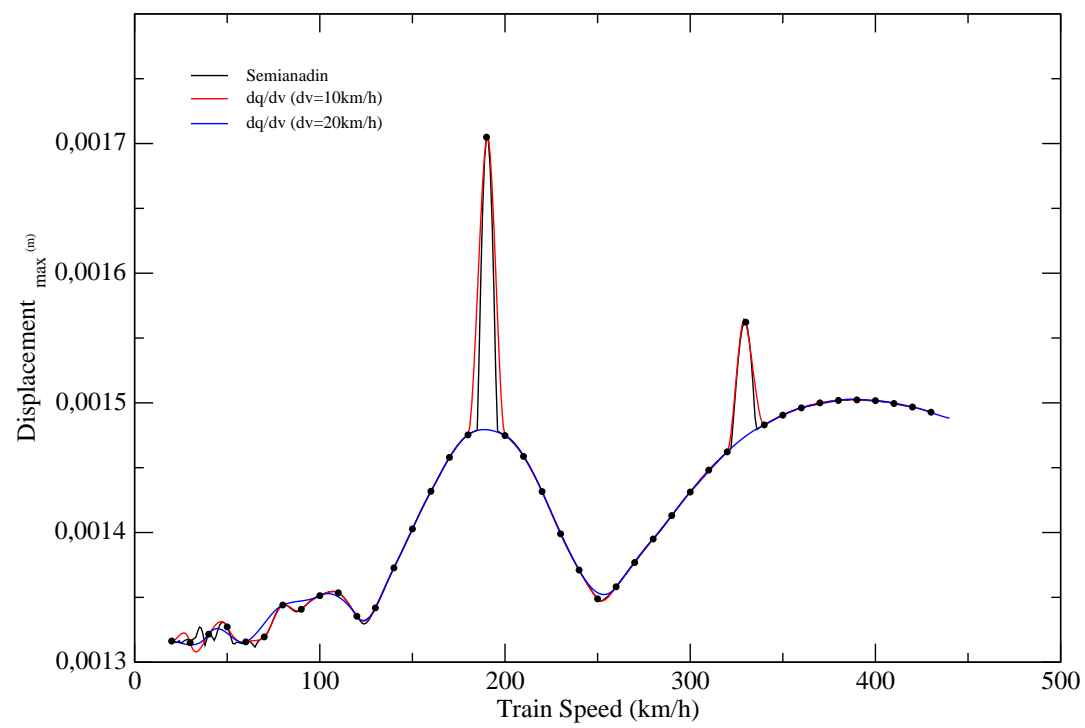

Figure 2.59: Train A1, post-process point 2. Effects of velocity stepping. 
Displacement envelope of train A1. Effect of $\Delta t$.

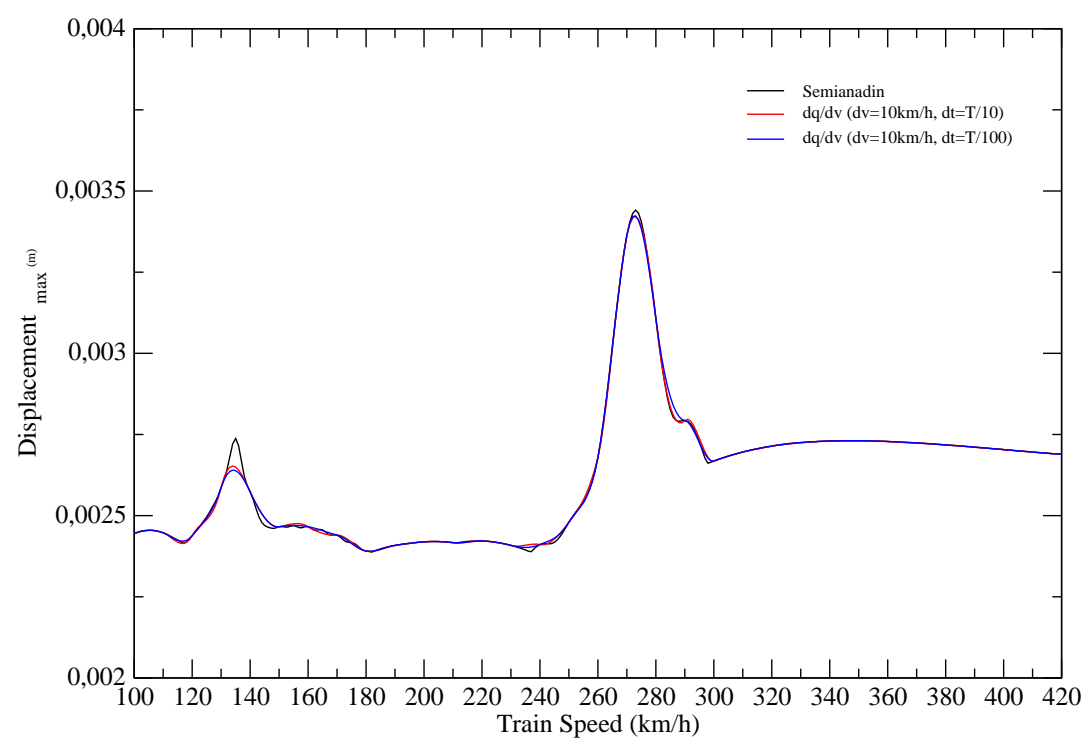

Figure 2.60: Train A1, post-process point 2. Effects of time stepping.

Displacement envelope of train A1. Rayleigh's damping.

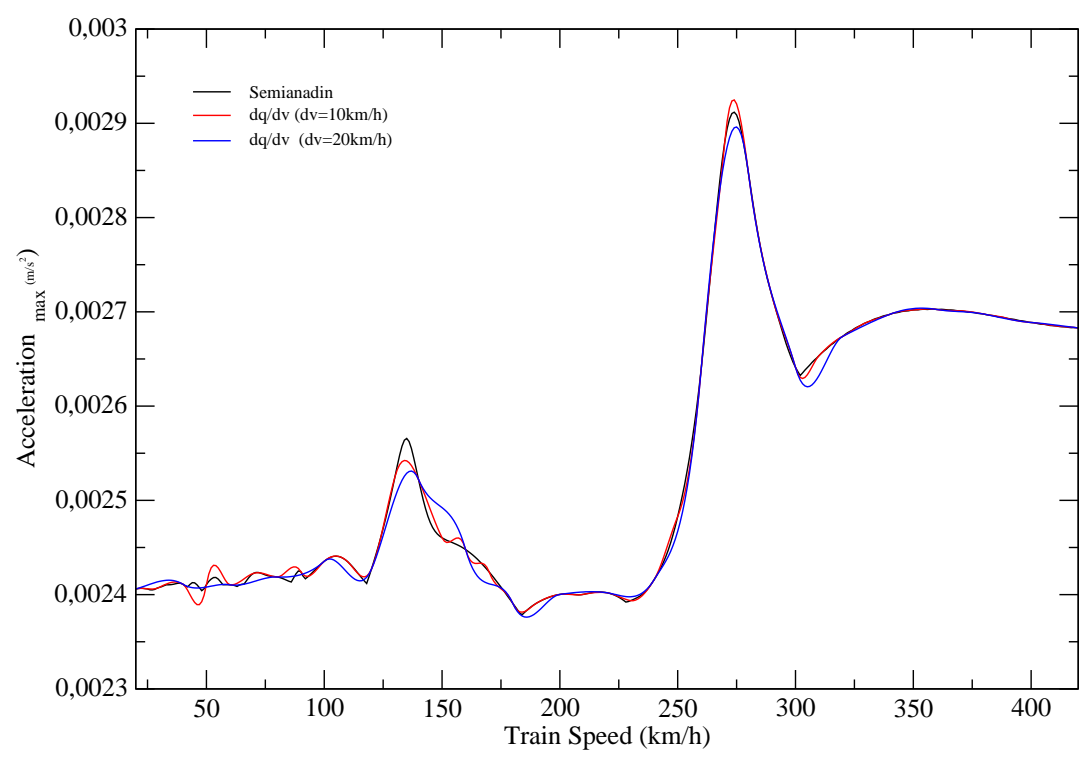

Figure 2.61: Train A1, post-process point 2. Displacement evaluation under Rayleigh damping. 
All trains displacement envelope.

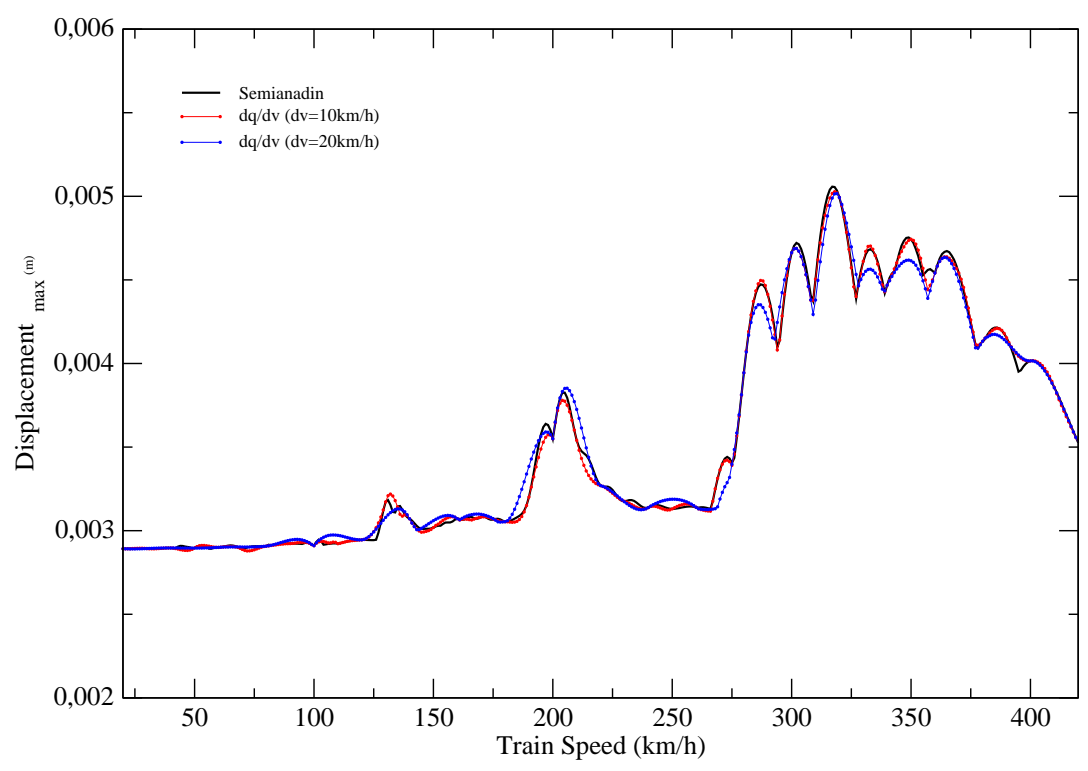

Figure 2.62: Displacement envelope on post-process point 2.

Conclusion. Errors and time of calculus.

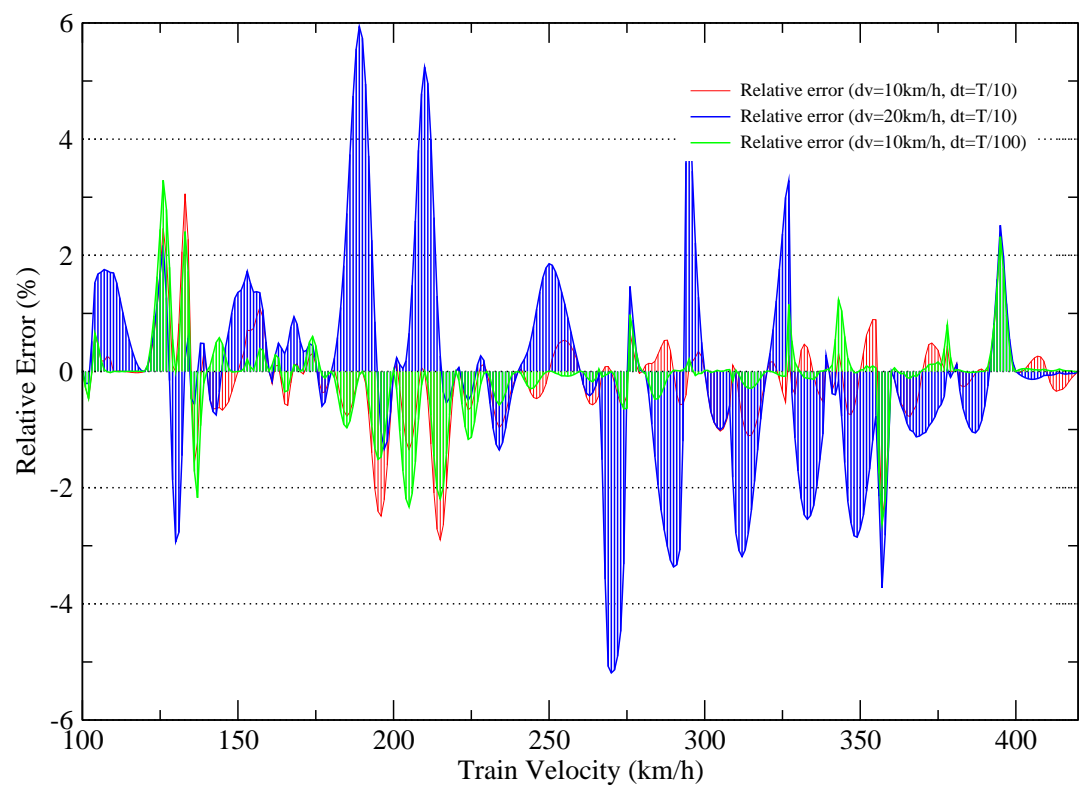

Figure 2.63: Relative error on displacement envelopes of point 2.

In terms of time of calculus, the time required to obtain the curves of the figure 2.63 are: 
- Semianalytic solution $\left(\Delta \mathrm{v}=1 \mathrm{~km} / \mathrm{h}, \Delta \mathrm{t}=T_{1} / 10\right)=1364 \mathrm{~s}$

- $\mathrm{dq} / \mathrm{dv}$ solution $\left(\Delta \mathrm{v}=10 \mathrm{~km} / \mathrm{h}, \Delta \mathrm{t}=T_{1} / 10\right)=159.67 \mathrm{~s}$ (reduction of $11.7 \%$ )

- $\mathrm{dq} / \mathrm{dv}$ solution $\left(\Delta \mathrm{v}=10 \mathrm{~km} / \mathrm{h}, \Delta \mathrm{t}=T_{1} / 100\right)=1561.45 \mathrm{~s}$ (reduction of $114.48 \%$ )

- $\mathrm{dq} / \mathrm{dv}$ solution $\left(\Delta \mathrm{v}=20 \mathrm{~km} / \mathrm{h}, \Delta \mathrm{t}=\mathrm{T}_{1} / 10\right)=87.28 \mathrm{~s}$ (reduction of $6.40 \%$ )

The study of this two magnitudes defines the utility of this proposed method as an approximated analysis in the study of different alternatives during the design of a railway bridge. With this, depending on the accuracy that the designer needs in relation with the phase of design that he is facing, he will be able to analyze different alternatives without spending long time of calculus in each case. 


\section{CHAPTER 3}

\section{Method 2: Hilbert's transformation.}

\subsubsection{Introduction}

Hilbert transformation, named by its creator David Hilbert (1862-1943), is a linear operator that attaches a holomorphic function to other in such a way they together create a so called strong analytical signal. The strong analytic signal can be written with an amplitude and a phase where the derivative of the phase can be identified as the instantaneous frequency.

Through its analytical expression, it is possible to demodulate the signals so that one can determine its envelopes without rapid oscillations. This characteristic is very interesting in the dynamic analysis because the aim of all the process is to obtain a general envelope of the maximum responses of the structure, because of this, all the information about rapid oscillations are useless but the maximum values which are well determined by this envelope. Besides, the curve obtained by the module of the analytical Hilbert signal is smoother what permits its definition by a higher time stepping. By this way, this method treats the other variable, time sampling, focusing its optimization in time of calculus by minimizing the determination of the maximum values of the time series by Hilbert transformation. Finally, a window filter is implemented too in order to reduce the time in the obtaining of the Hilbert transformation, taking advantage of the local behavior of this transformation is possible to avoid the whole integration in the time domain without losing too much information.

\section{Theorethical concepts}

\section{Main properties}

1. The HT is a linear operator, so if $\alpha_{1}$ and $\alpha_{2}$ are arbitrary (complex) scalars, and $f_{1}$ and $f_{2}$ are varying signals, then:

$$
h\left[\alpha_{1} \cdot f_{1}+\alpha_{2} \cdot f_{2}\right]=\alpha_{1} \cdot \tilde{f}_{1}+\alpha_{2} \cdot \tilde{f}_{2}
$$

2. The HT of an HT yields the original function with the opposite sign, hence it carries out a shifting of the initial signal by $-\pi$.

3. The power (or energy) of a signal and its HT are equal.

4. A function and its HT are orthogonal over an infinite interval:

$$
\int_{-\infty}^{\infty} f(t) \cdot \widetilde{f}(t) d t=0
$$




\section{The Hilbert transform of multiplication}

In practice its very common the need for the transformation of a product of functions. The equation:

$$
H\left[f_{\text {slow }}(t) \cdot f_{\text {fast }}\right]=f_{\text {slow }}(t) \cdot \tilde{f}_{\text {fast }}
$$

it is called the Bedrosian identity (product theorem). It states that the HT of the product of a lowpass and highpass signals with non over lapping spectra is defined by a product of the lowpass signal and the HT of the highpass signal.

\section{Analytic signal}

A complex signal whose imaginary part is the HT of the real part is called an analytic or quadrature signal. Such a signal is a two-dimensional signal whose value at some instant in time is specified by two parts, a real part and an imaginary one:

$$
F(t)=f(t)+i \cdot \tilde{f}(t)
$$

For each integral transform, there will be other relation which converts the transformed function back into the original function. At the same time this relation usually is an integral transform although sometimes it may be written in terms of algebraic operations only. In order to return from a complex form of the analytic signal $F(t)$ back to the real function $f(t)$, one has to use a substitution:

$$
f(t)=\frac{1}{2} \cdot[F(t)+\bar{F}(t)]
$$

where $\bar{F}(t)$ is the complex conjugate signal of $\mathrm{F}(\mathrm{t})$

\section{Polar notation}

An analytic signal has a geometrical representation in the form of a phasor rotating in the complex plane. A phasor can be viewed as a vector at the origin of the complex plane having a length $\mathrm{A}(\mathrm{t})$ and an angle, or an angular position, $\psi(\mathrm{t})$. The projection on the real axis is the initial real signal and it is described by $\mathrm{f}(\mathrm{t})=\mathrm{A}(\mathrm{t}) \cdot \cos \psi(\mathrm{t})$. Using a traditional representation of the analytic signal is its trigonometric or exponential form:

$$
F(t)=|F(t)|[\cos \psi(t)+i \cdot \operatorname{sen} \psi(t)]= \pm A(t) \cdot e^{i \psi(t)}
$$

one can determine its instantaneous amplitude (envelope, magnitude, modulus):

$$
A(t)= \pm|F(t)|= \pm \sqrt{f^{2}(t)+\widetilde{f}^{2}(t)}
$$

and its instantaneous phase: 


$$
\psi(t)=\arctan \frac{\tilde{f}(t)}{f(t)}
$$

\section{Hilbert envelope}

The form or the shape of the variation of the instantaneous amplitude is called a wave envelope. If the waveform is a pure harmonic with varying positive and negative signal values, the relationships between peak-to-peak amplitude and the root mean square (standard deviation) values are fixed and known, as they are for any continuous periodic wave:

$$
\text { Peak }- \text { to }- \text { peak }=2 \sqrt{2} \text { RMSwhe }
$$

where

$$
R M S=\left[\delta t^{-1} \int_{0}^{\delta t} x^{2}(t) d t\right]^{(1 / 2)}
$$

However, this is not true for an arbitrary waveform which may or may not be periodic or continuous. In a general case an amplitude of the oscillation $A(t)$-as a magnitude of the complex analytical signal- varies with time. As initial signal and its envelope have common tangents at points of contact, but the signal never crosses the envelope. The plus sign of the root square corresponds to the upper positive envelope $\grave{A}(\mathrm{t})$, the minus sign corresponds to the lower negative envelope $-\mathrm{A}(\mathrm{t})$, so they are always in the anti-phase relation. An envelope function contains an important information about the signal energy. It is known that power is a time average of energy (energy per unit time):

$$
\text { Power }=T^{-1} \int_{0}^{T} x^{2}(t) R M S^{2}
$$

Because the power in the HT pair projection is equal to the power in the original signal, the envelope is precisely twice the signal power. By using the HT, the rapid oscillations can be removed from the amplitude modulated signal to produce a direct representation of the slow envelope alone. The spectrum of the complex envelope can be obtained by shifting an initial signal spectrum to the left towards the origin of axes.

For more information see [26] or [50].

\subsubsection{Application to Semi-Analytic methodology}

The Hilbert transform $\tilde{f}(t)$ of a function $\mathrm{f}(\mathrm{t})$ is defined by the convolution between the Hilbert transformer $\frac{1}{(\pi t)}$ and the function $\mathrm{f}(\mathrm{t})$ :

$$
H(x(t))=\widehat{f}(t)=\frac{1}{\pi} \int_{-\infty}^{\infty} \frac{f(\eta)}{t-\eta} \partial \eta
$$

Because of the possible singularity at $t=\eta$, the integral has to be considered as a Cauchy principal value. The HT of the real-valued function $\mathrm{f}(\mathrm{t})$ extending from $-\infty$ to $+\infty$ is a realvalued function $\widetilde{f}(t)$ defined by 3.12. Physically, the HT is an equivalent to a special kind 
of a linear filter, where all the amplitudes of the spectral components are left uncharged, but their phases are shifted by $-(\pi / 2)$. Thus, the HT representation $\tilde{f}(t)$ of the original function is the convolution integral of $\mathrm{f}(\mathrm{t})$ with $(\pi \cdot t)^{-1}$, written as $\tilde{f}(t) \mathrm{x}(\mathrm{t}) \cdot(\pi t)^{-1}$. The impulse response function of the ideal HT is shown in figure 3.1, (a), as well as the module and the phase characteristic of the HT transfer function, (b) and (c). It is clear that the HT of a time-domain signal $\mathrm{f}(\mathrm{t})$ is another time-domain signal $\widetilde{f}(t)$ and if $\mathrm{f}(\mathrm{t})$ is real-valued, then so is $\tilde{f}(t)$.

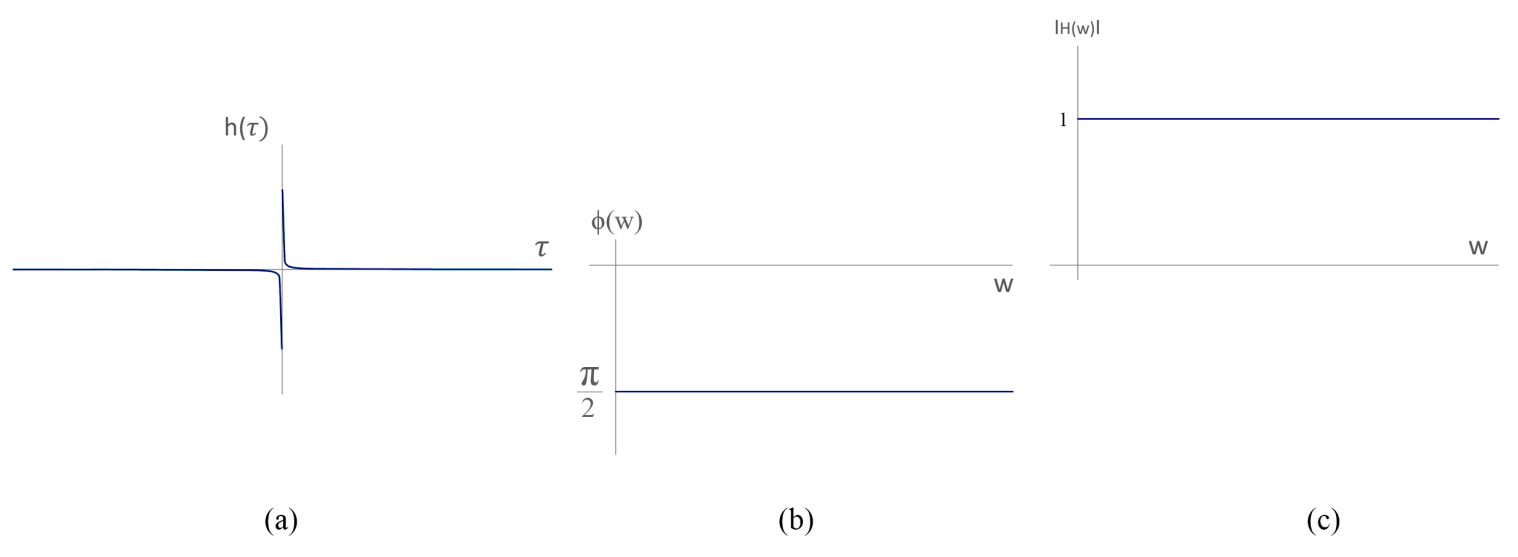

Figure 3.1: (a)Impulse response function of the ideal HT; (b)Module of the HT transfer function; (c) Phase of the HT transfer function.

In our case, the Semi-Analytic solution is defined by:

$$
q(t)=q p(t)+q h(t)
$$

\section{Hilbert transformation of the homogeneous part:}

\section{Complete Hilbert transformation:}

The homogeneous part of the semi-analitic solution is defined by:

$$
q h(t)=e^{-\xi \cdot w n \cdot t} \cdot(A n \cdot \cos (w d \cdot t)+B n \cdot \sin (w d \cdot t))
$$

The complete Hilbert transformation is obtained by applying the general formulation. It is a improper integral, therefore, it is necessary to define a parametric integration around the pole in order to solve the principal Cauchy value by a limit:

$$
\widetilde{q h}(t)=\frac{1}{\pi} \cdot \int_{-\infty}^{\infty} \frac{q h(\eta)}{(t-\eta)} \partial \eta
$$

In this point, it is remarkable the great advange of this semi-analitic methodology compared to the traditional ste-by-step methods, the Semi-Analitic solution is analitic in the time domain. Because of this, it is possible to obtain the exact analytic Hilbert transformation, 
what would be impossible in classical methods which only can obtain it is transformation once the response is obtained and by numerical approximations.

$$
\begin{gathered}
\tilde{q h}(t, p)=\frac{1}{2} e^{-i \cdot t \cdot w d-t \cdot w n \cdot \xi}(-(A n+i \cdot B n) * \text { ExpIntegralE } i[-i(p-t)(w d-i \cdot w n \cdot \xi]- \\
-\quad(A n-i \cdot B n) e^{2 \cdot i \cdot t \cdot w d} \text { ExpIntegralE } i[i(p-t)(w d+i \cdot w n \cdot \xi])
\end{gathered}
$$

Where ExpIntegralEi is an integral function defined by:

$$
\text { ExpIntegralEi }(x)=\int_{1}^{\infty} \frac{e^{-t x}}{t} d t
$$

And its graphic representation is 3.2. For more information see [51].

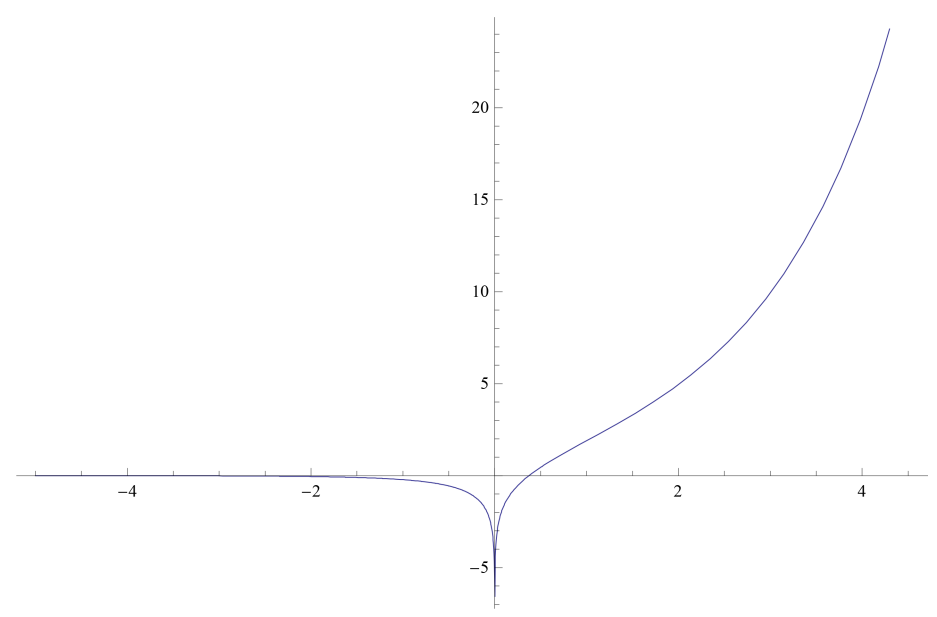

Figure 3.2: ExpIntegralEi function

It is possible to obtain the limits in the infinity, nevertheless, it would be enough by defining an enough wide window of integration. One the transformation is obtained, it is possible to define the analytical signal:

$$
\overline{q h}(t)=q h(t)+i \cdot \widetilde{q h}(t)
$$

And its module:

$$
|\overline{q h}(t)|=\sqrt{q h(t)^{2}+\widetilde{q h}(t)^{2}}
$$

defines the Hilbert envelope. One example of this transformation is represented in figure 3.3: 


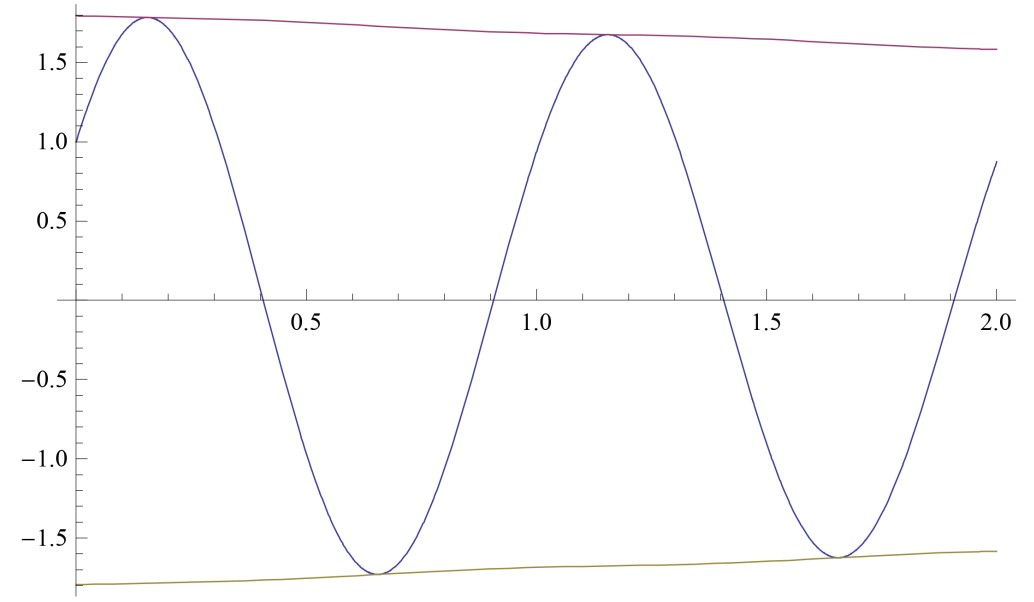

Figure 3.3: Complete Hilbert transformation of the homogeneous part of Semi-Analitic solution.

Hilbert transformation of the product: lowpass and highpass signals

Taking into consideration the product theorem exposed above, its easy to distinguish the two different behaviors of the exponential part and the rest of the expression of $\mathrm{qh}(\mathrm{t})$ :

$$
q h(t)=q h_{\text {slow }}(t) \cdot q h_{f a s t}(t)=e^{-\xi \cdot w n \cdot t} \cdot \varphi(t)
$$

Here, the exponential factor has a slow variation while the fluctuations of $\varphi(t)$ are much faster. According to the product theorem, it can defined as the multiplying of the slow variation terms and the Hilbert transformation of the fast varying terms:

$$
\widetilde{q h}(t)=e^{-\xi \cdot w n \cdot t} \cdot \frac{1}{\pi} \cdot \int_{-\infty}^{\infty} \frac{\varphi(\eta)}{(t-\eta)} \partial \eta
$$

Once again, the solution of the latter integral results, defining the parametric integration around the pole in order to solve the principal Cauchy value by a limit:

$$
\begin{gathered}
\widetilde{\varphi}(t, p)=\int_{-\infty}^{\infty} \frac{q h(\eta)}{(t-p)} \partial p \\
\widetilde{\varphi}(t, p)=\operatorname{SinIntegral}(w d \cdot(p-t)) \cdot(A n \cdot \sin (t \cdot w d)-B n \cdot \cos (t \cdot w d))- \\
-\operatorname{CosIntegral}(w d \cdot(p-t)) \cdot(A n \cdot \cos (w d \cdot t))+B n \cdot \sin (w d \cdot t))
\end{gathered}
$$

Where CosIntegral and SinIntegral are two integral functions defined by:

$$
\begin{gathered}
\text { CosIntegral }(x)=-\int_{x}^{\infty} \frac{\cos (t)}{t} d t \\
\text { SinIntegral }(x)=\int_{0}^{x} \frac{\sin (t)}{t} d t
\end{gathered}
$$


And their graphic representations are 3.4 and 3.5 respectively. For more information see [51].

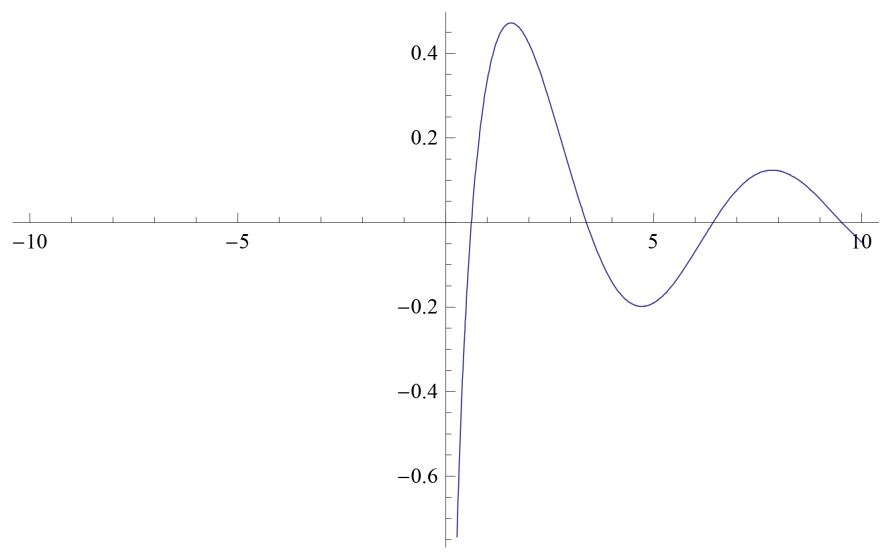

Figure 3.4: CosIntegral function

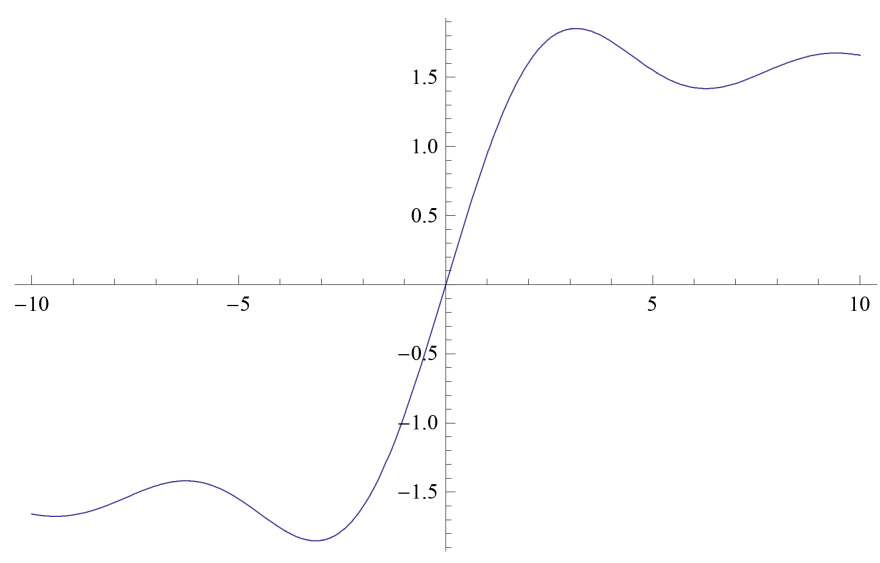

Figure 3.5: SinIntegral function

The pole is defined in $\mathrm{p}=\mathrm{t}$ so the determination of the principal Cauchy value is as follows:

$$
\begin{aligned}
& \tilde{q h}(t)=\frac{1}{\pi} \cdot e^{-\xi \cdot w n \cdot t} \cdot\left[\int_{-\infty}^{t} \frac{\varphi(\eta)}{(t-\eta)} \partial \eta+\int_{t}^{\infty} \frac{\varphi(\eta)}{(t-\eta)} \partial \eta\right]= \\
= & \frac{1}{\pi} e^{-\xi \cdot w n \cdot t} \cdot\left(\lim _{p \rightarrow \infty} \widetilde{\varphi}(t, p)-\lim _{p \rightarrow-\infty} \widetilde{\varphi}(t, p)\right)
\end{aligned}
$$

In this case, it is also possible to obtain the limits in the infinity, nevertheless, it would be enough by defining an enough wide window of integration. One the transformation is obtained, it is possible to define the analytical signal: 


$$
\overline{q h}(t)=q h(t)+i \cdot \tilde{q h}(t)
$$

And its module:

$$
|\overline{q h}(t)|=\sqrt{q h(t)^{2}+\widetilde{q h}(t)^{2}}
$$

defines the Hilbert envelope. One example of this transformation is represented in figure 3.6:

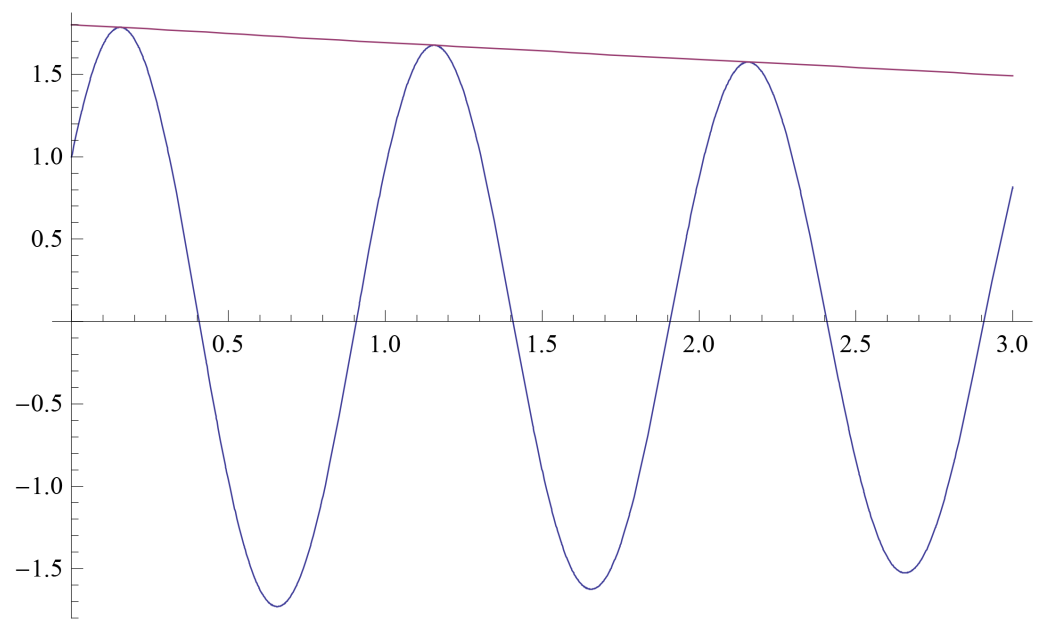

Figure 3.6: Hilbert transformation of the homogeneous part of Semi-Analitic solution based on the product theorem.

Approximation to Hilbert transformation of the homogeneous part.

Despite being able to obtain the exact analitical Hilbert transformation, as well as the demodulated solution by the application of the product theorem, the use of the integral functions CosIntegral, SinIntegral and ExpintetralEi difficults the process and their evaluation requires a great computational cost. However, it is easy to check the great local character of this transformation, thus, it is possible to define the Hilbert transformation of the homogeneous part as the sum of the transformation of the separate elements of the highpass part of the solution:

$$
\widetilde{q h}(t)=e^{-\xi \cdot w n \cdot t} \cdot(A n \cdot \widetilde{\cos }(w d \cdot t)+B n \cdot \widetilde{\sin }(w d \cdot t))
$$

Where:

$$
\begin{aligned}
& \widetilde{\cos }(w d \cdot t)=-\sin (w d \cdot t) \\
& \widetilde{\sin }(w d \cdot t)=\cos (w d \cdot t)
\end{aligned}
$$

Thus, the Hilbert transformation has the form: 


$$
\widetilde{q h}(t)=e^{-\xi \cdot w n \cdot t} \cdot(-A n \cdot \sin (w d \cdot t)+B n \cdot \cos (w d \cdot t))
$$

This new expression is proved to be accuracy enough (3.7) and the expression is the simplest, besides, it presents the same form of the original Semi-Analitic solution what makes very easy its implementation.

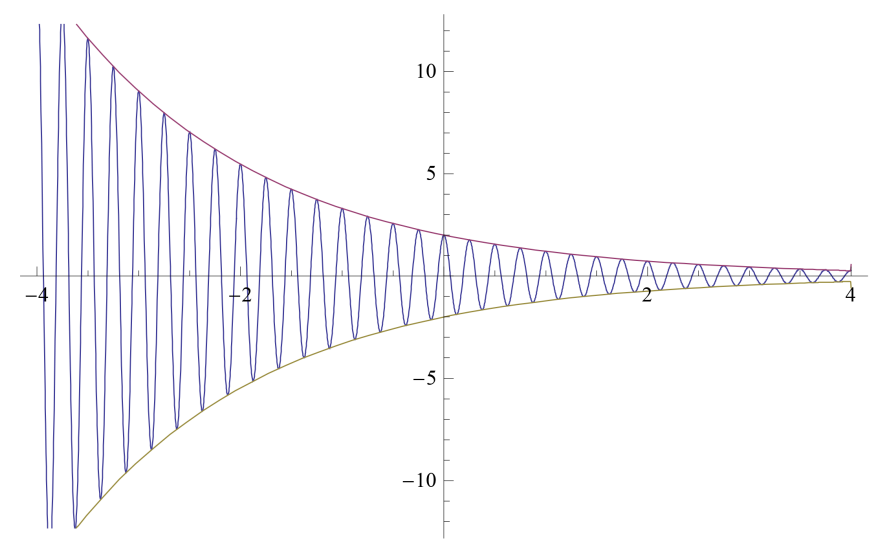

Figure 3.7: Local Hilbert transformation of the homogeneous Semi-Analitic solution.

Hilbert transformation of the particular solution:

The particular solution in acceleration has a form as follows:

$$
q p(t)=2 \cdot a_{2} \cdot v^{2}+6 \cdot a_{3} \cdot c^{3} \cdot t
$$

In order to obtain the Hilbert transformation, the scheme is the same integrating with a parameter p:

$$
\widetilde{q p}(t, p)=\frac{1}{\pi} \int \frac{q p(p)}{t-p} \partial p
$$

Resulting:

$$
\begin{aligned}
& \widetilde{q p}(t, p)=\left(\frac{1}{\pi}\right) \cdot\left(-\frac{1}{6} \cdot(p-t) \cdot\left(6 \cdot a_{1}+6 \cdot a_{2}+3 \cdot a_{3} \cdot p^{2}+5 \cdot a_{3} p t+11 a_{3} t^{2}\right)-\right. \\
- & \left.\left(a_{0}+t \cdot\left(a_{1}+a_{2}+a_{3} \cdot t^{2}\right)\right) \log |t-p|\right)
\end{aligned}
$$

And, as above, to obtain the principal Cauchy value it is necessary to solve the improper integral:

$$
\begin{aligned}
& \widetilde{q p}(t)=\frac{1}{\pi} \cdot e^{-\xi \cdot w n \cdot t} \cdot\left[\int_{-\infty}^{t} \frac{\varphi(\eta)}{(t-p)} \partial p+\int_{t}^{\infty} \frac{\varphi(p)}{(t-\eta)} \partial p\right]= \\
= & \frac{1}{\pi} e^{-\xi \cdot w n \cdot t} \cdot\left(\lim _{p \rightarrow \infty} \widetilde{\varphi}(t, p)-\lim _{p \rightarrow-\infty} \widetilde{\varphi}(t, p)\right)
\end{aligned}
$$




\subsubsection{Validation}

\subsubsection{Window filter}

As seen until now, Hilbert envelope is smoother than the time series of response, thus its determination permits the definition of higher time steppings. However, its necessary to integrate the response along the whole bridge, this fact makes this method slower than the traditional Semi-Analytic method not because of the time stepping but the integration of the Hilbert transformation. Nevertheless, although the Hilbert transformation is defined along the whole domain of the function, the main contribution is focused nearby the pole of integration and its importance decreases with the distance from it. Therefore, a filter based on this concept has been developed, a window filTer. This filter consists on integrating the Hilbert transformation only along a fixed distance, $\mathrm{d}$, from the pole, taking into consideration the distance between the axes of the train $(\approx 10 \mathrm{~m})$, and neglecting the contribution of the rest of the domain.

$$
\widetilde{q h}_{\text {windowfilter }}(t)=\frac{1}{\pi} \cdot \int_{t-d / 2}^{t+d / 2} \frac{q h(\eta)}{(t-\eta)} \partial \eta
$$

This filter implies a loss of the demodulation of the signals, however, as seen before the Hilbert envelope is tangent to the original signal on the maximum values and it is always above it, hence, this loss will only approximate this two curves. From now on, the investigation is focused on the application of this methodology to the same tests analyzed with the latter methodology, dqd/dv, analyzing the minimum windows to obtain successful results and the actual reduction in time of calculus.

\subsubsection{Validation with numeric approximations}

In order to validate the two proposed methodologies, complete Hilbert transformation and window filter, they both have been compared with the numerical one obtained by MATLAB r2000 applied to the time series of the response of the bridge described in Eurocode 1. In this structure, the obtained Hilbert transformation did not present a so effective demodulation as in the calibration test, that is why it was necessary to check whether it could be a mistake or a limitation of the proposed method or simply the Hilbert transformation is no so capable to full demodulate the response of tougher structures. 


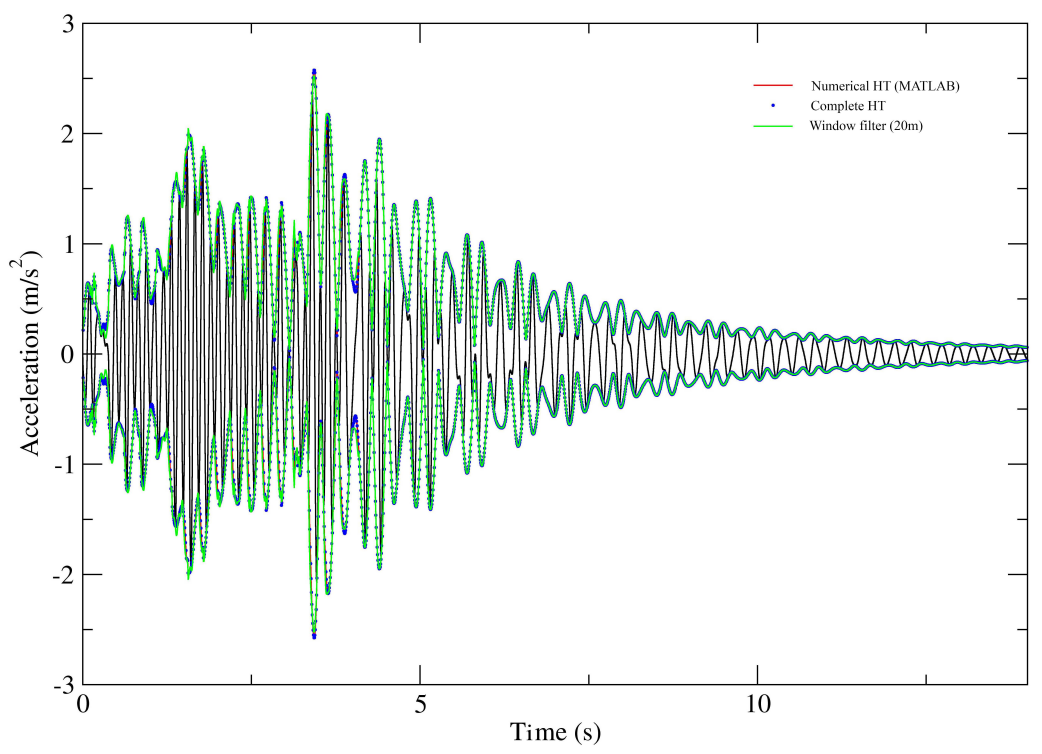

Figure 3.8: Acceleration time serie by complete Hilbert, window filter and numerical transformation of the eurocode 1 train under train A1 of HSML series and $v=420 \mathrm{~km} / \mathrm{h}$.

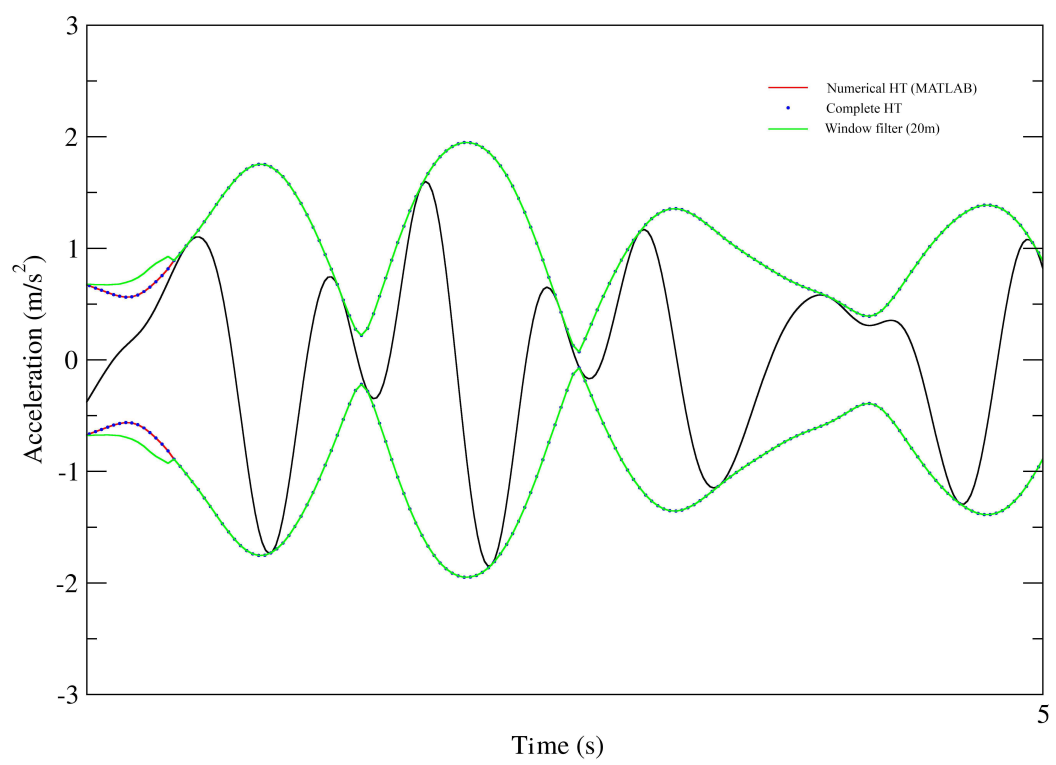

Figure 3.9: Detail of acceleration time serie by complete Hilbert, window filter and numerical transformation of the eurocode 1 train under train A1 of HSML series and v=420km/h. 


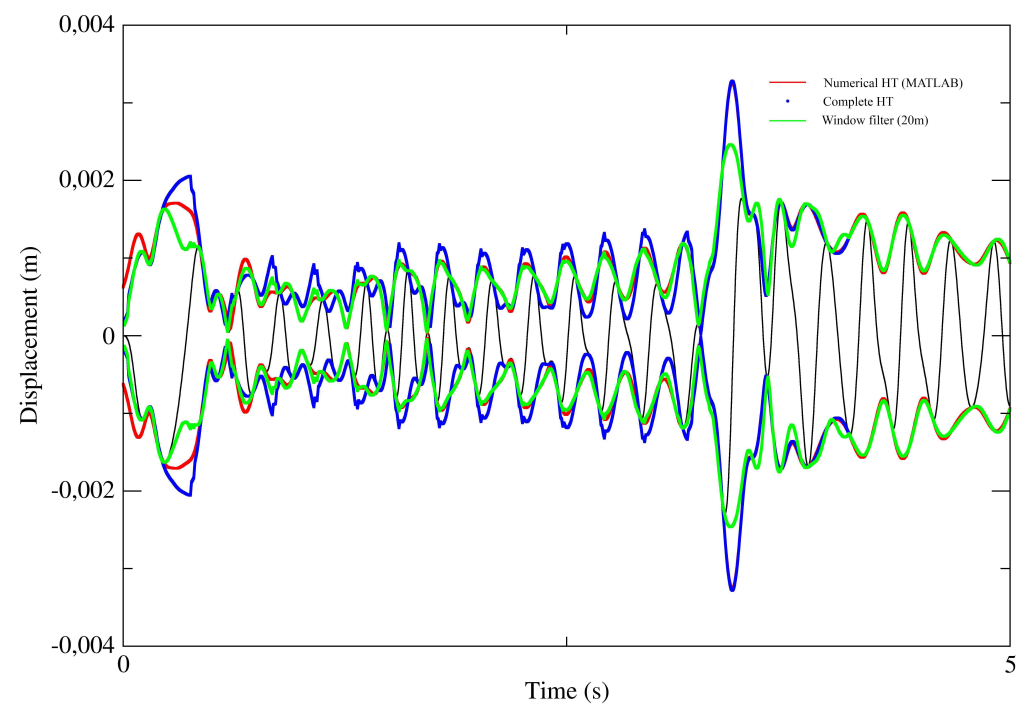

Figure 3.11: Detail of displacement time serie by complete Hilbert, window filter and numerical transformation of the eurocode 1 train under train A1 of HSML series and v=420km $/ \mathrm{h}$.

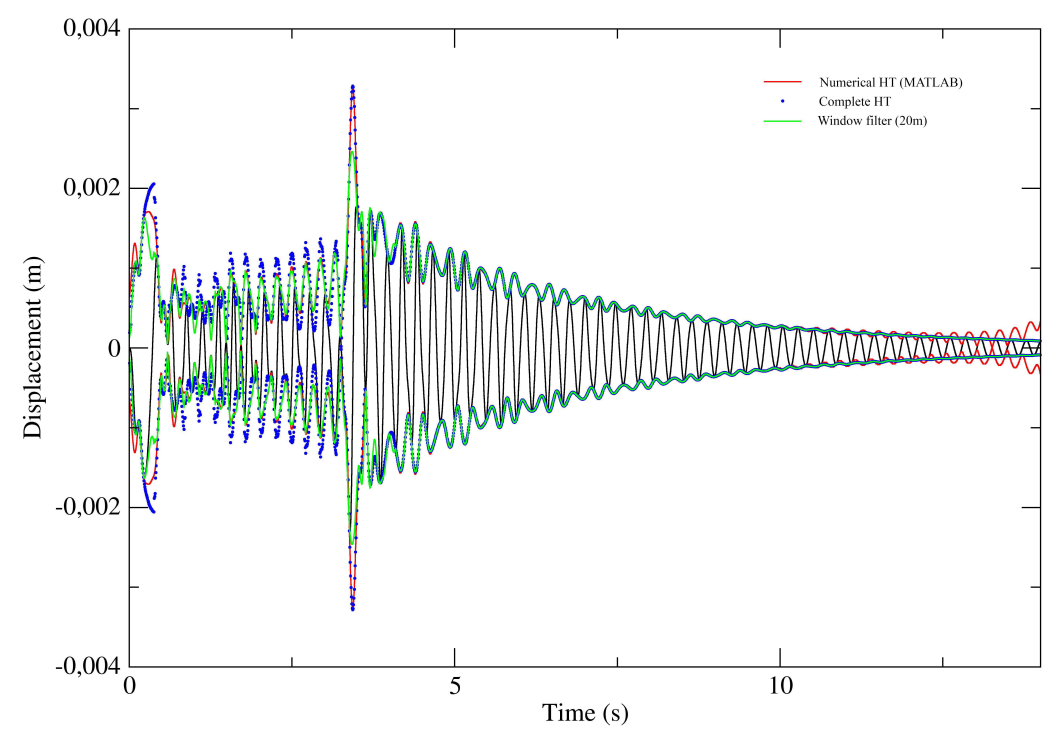

Figure 3.10: Displacement time serie by complete Hilbert, window filter and numerical transformation of the eurocode 1 train under train A1 of HSML series and v=420km $/ \mathrm{h}$.

The transformation are applied to the time series of acceleration and displacement. As can be see in figures 3.8, 3.9, 3.10 and 3.11, the Hilbert transformation coincide in almost all the domain, even the numerical Hilbert transformation presents worse results in high velocities producing ficticious excitations. In spite of the fact that the demodulation is not complete and the residual fluctuating behaviors is not negligible, the transformation is much smoother than the original one what allows the definition of higher time stepping in order to collect the mamximum values of the series. Besides, it is noticed that the results of the 
complete transformation and the window filer coincide in great part of the domain, what confirms the local character of this transformation and the utility of the proposed filter.

\subsection{Tests}

In this section, some of the studied tests for $\mathrm{dq} / \mathrm{dv}$ methodology are retaken in order to denote the properties and applicability of the defined Hilbert's transformation in is complete and window-filtered version.

\subsubsection{Simply supported beam (IAPF)}

First of all, it is studied the simply supported beam studied in the Spanish standard IAPF [18]. It has been determined the response of the structure to several load cases in terms of time series and maximum envelopes.

Response to one single load

The first load case is defined as a single load of $100 \mathrm{kN}$ crossing the structure at $150 \mathrm{~km} / \mathrm{h}$. Taking advantage of the simplicity of the problem, the results show very clearly the geometric properties of the Hilbert's envelope curve.

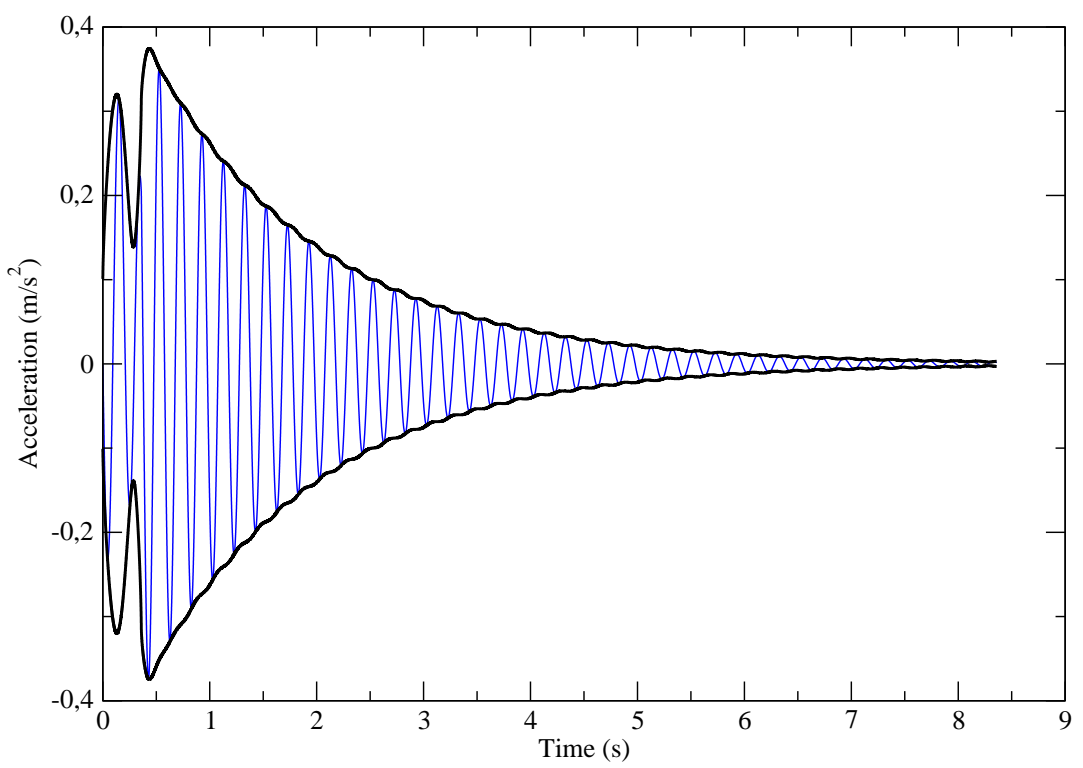

Figure 3.12: Semi-Analytic time series of acceleration and its complete Hilbert's transformation of mid span of simply supported beam (IAPF) under a single load of $100 \mathrm{kN}$ at $150 \mathrm{~km} / \mathrm{h}$ 


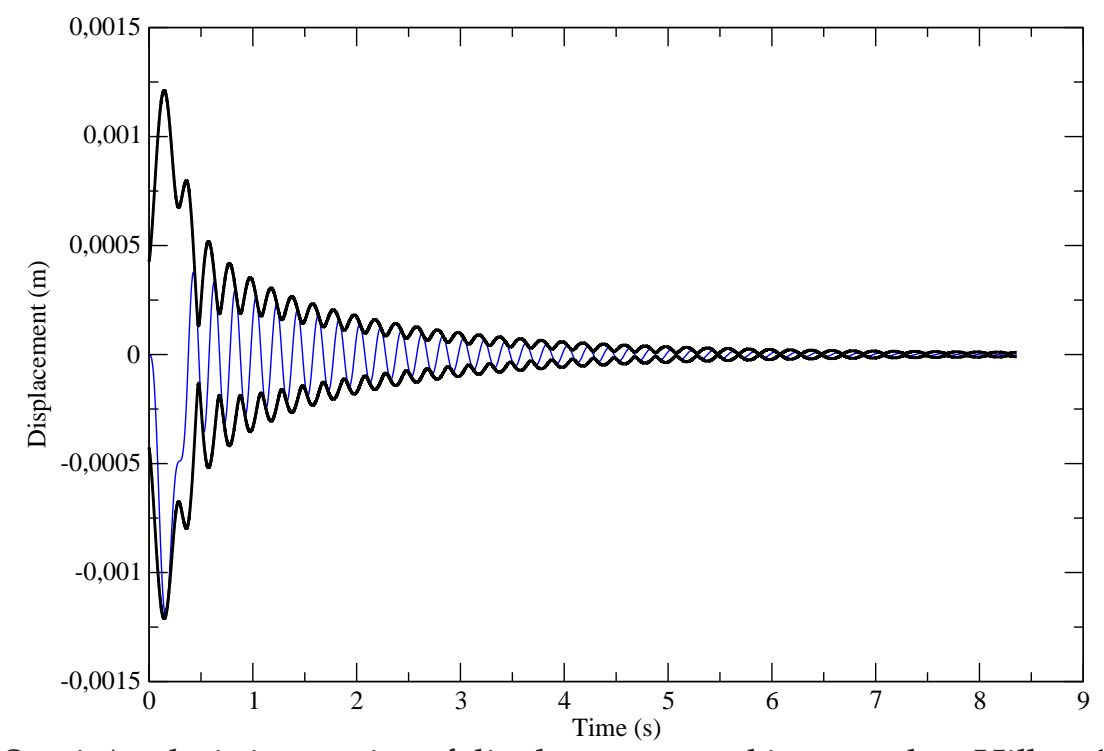

Figure 3.13: Semi-Analytic ime series of displacement and its complete Hilbert's transformation of mid span of simply supported beam (IAPF) under a single load of $100 \mathrm{kN}$ at $150 \mathrm{~km} / \mathrm{h}$

As seen in latter section, Hilbert's transformation allows the definition of the complex analytic signal and its module is called the Hilbert's envelope. Besides, Hilbert's transformation has the same power as the original signal but in difference of phase, this property provokes the demodulation of the original signal once they are combined in the module of the analytic signal, Hilbert's envelope. This effect is very clear in figure 3.12 where the fluctuating term is almost completely eliminated. In figure 3.13, it can be seen that the demodulation of Hilbert's envelope is not so effective as for acceleration. Nevertheless, the Hilbert's envelope is always above the original signal but tangent in some points. This last property can be seen more clearly maximum envelope analysis, 3.14 and 3.15, in which the envelope obtained by Hilbert's envelope always remains above the one obtained by classic Semi-Analytic method. 


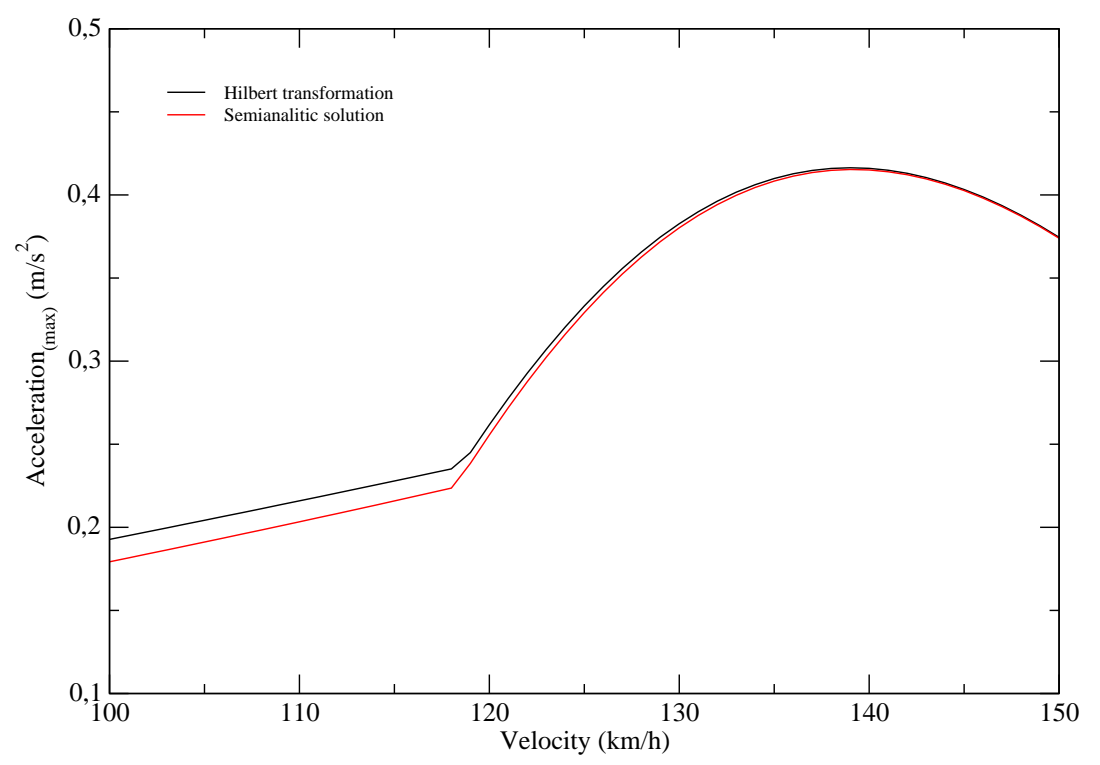

Figure 3.14: Acceleration envelope of mid span of simply supported beam (IAPF) under a single load o

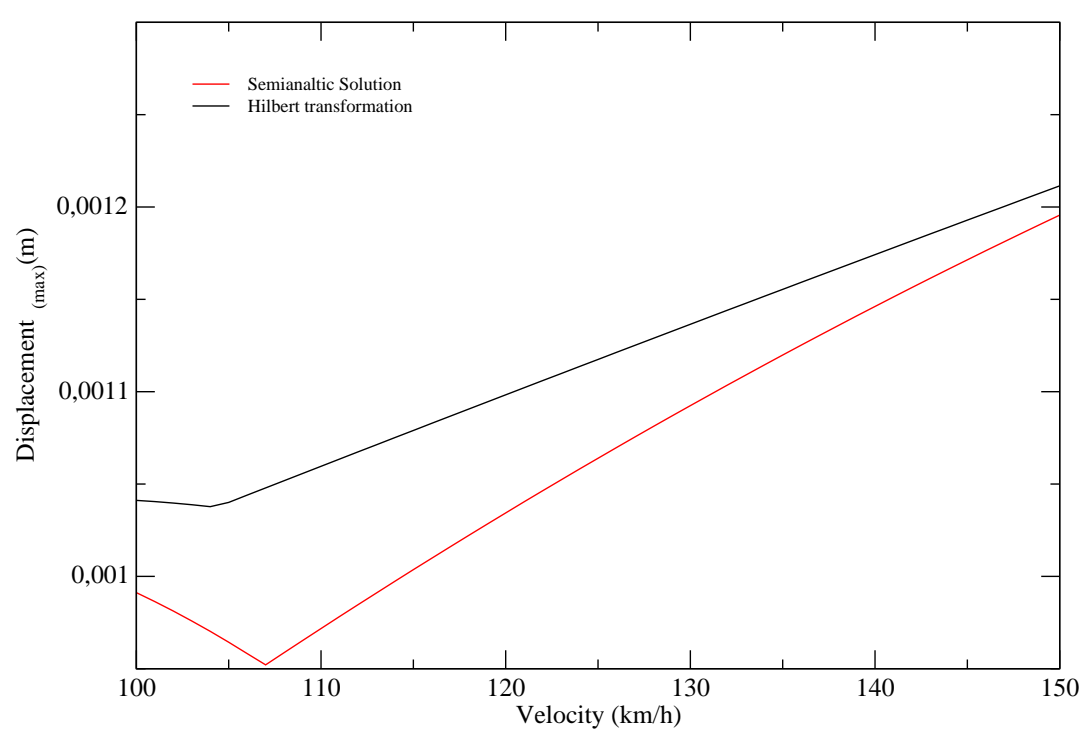

Figure 3.15: Displacement envelope of mid span of simply supported beam (IAPF) under a single load of $100 \mathrm{kN}$

\section{Response to train A1 from HSML series}

The second analyzed load case is first train of the HSML series. The more complex scheme of the loading the higher relevance of fluctuating behaviors, fact that can be seen in figures 3.16 and 3.17 in which the fluctuating behavior remains not negligible in the Hilbert's envelope. However, once again, the resultant signal keeps above the original signal but tangent in some points. 


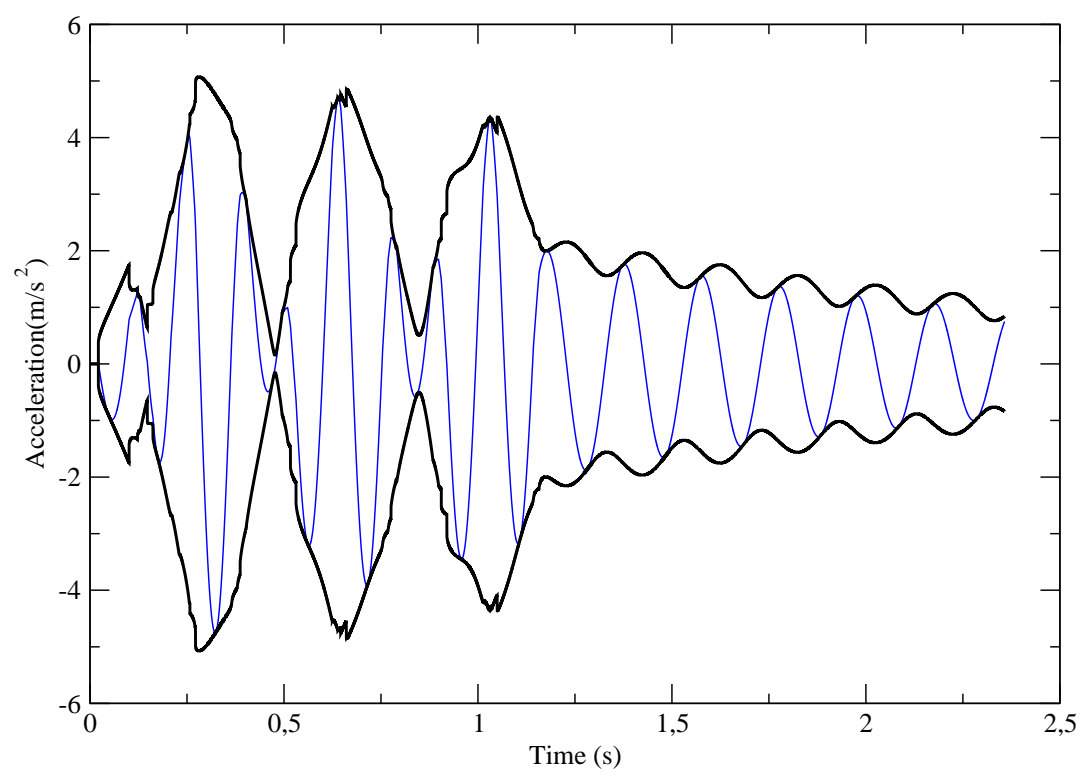

Figure 3.16: Time series of acceleration of mid span of simplv supported beam (IAPF) under the train A1

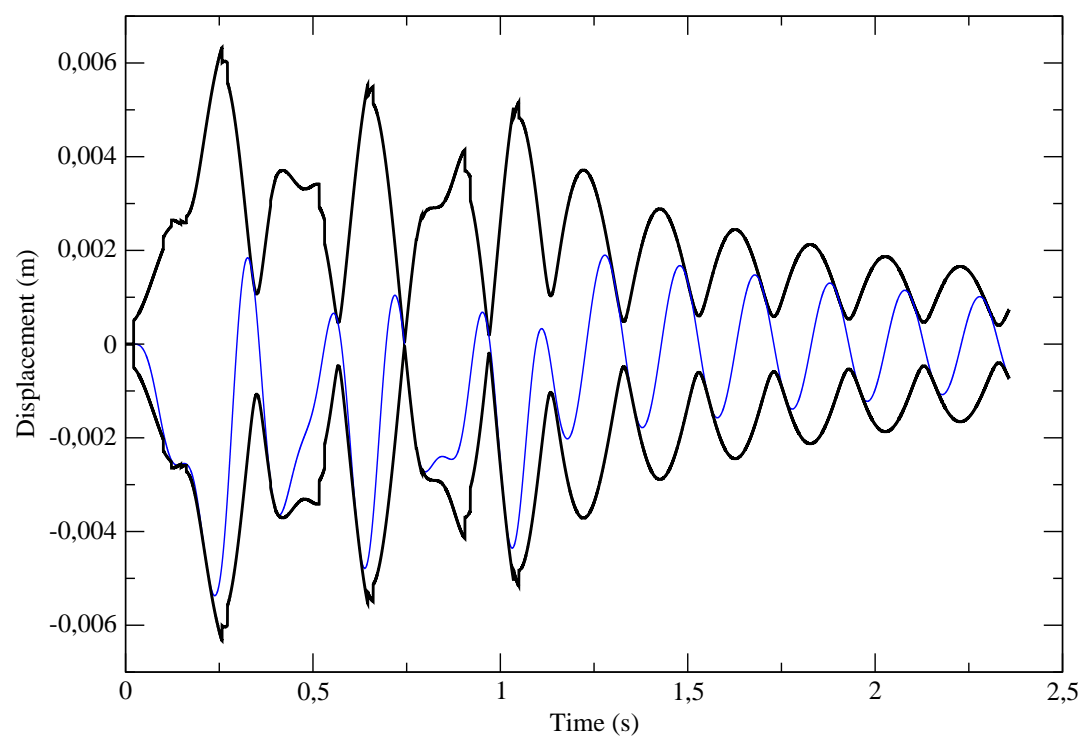

Figure 3.17: Time series of displacement of mid span of simply supported beam (IAPF) under the train A1 of the HSML series at $500 \mathrm{~km} / \mathrm{h}$

\section{Response to train ICE2}

Finally, the last load case is the train ICE2. It is studied because it is the chosen load case in the standard IAPF [18]. This train is composed by 56 axes and the structure is a simple $15 \mathrm{~m}$ length simply supported beam, hence, it was expected to obtain a highly fluctuating behavior. Because of this, as in latter examples, the demodulation of the Hilbert's envelope is limited as can be seen in figure 3.18. 


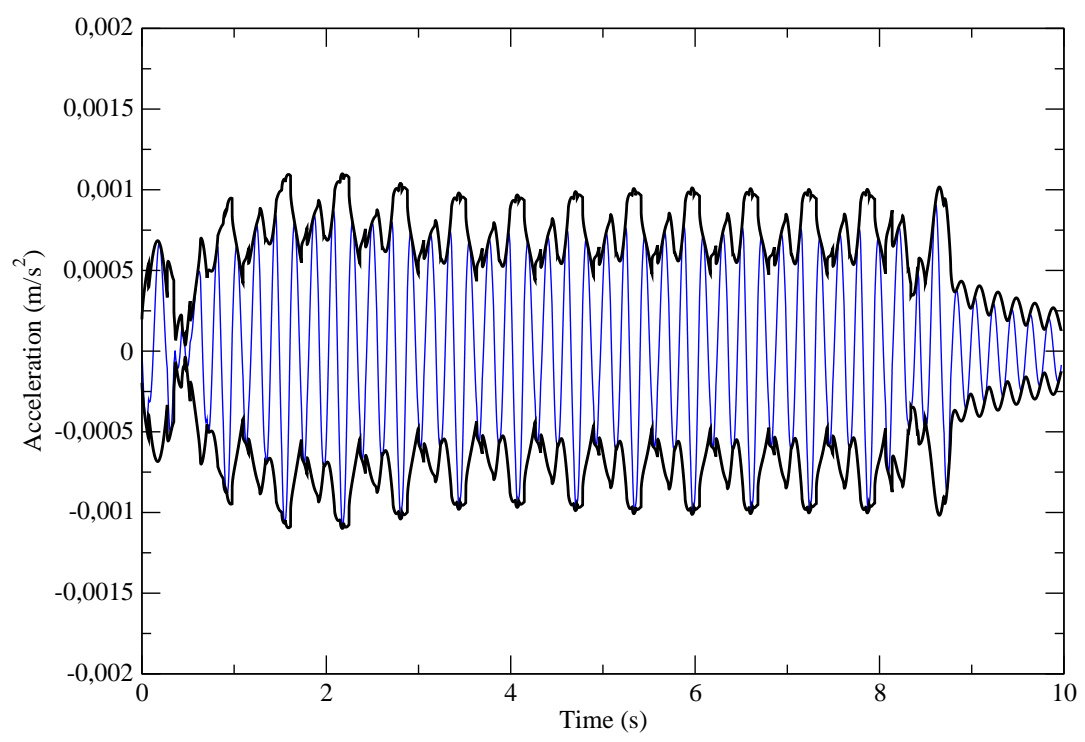

Figure 3.18: Time series of acceleration of mid span of simply supported beam (IAPF)under the train ICE2 at $150 \mathrm{~km} / \mathrm{h}$

\section{Effect of window filter}

Finally, the effect of window filter is analyzed. To that effect, the first load case of $100 \mathrm{kN}$ running at $150 \mathrm{~km} / \mathrm{h}$ is chosen because of its clearity in its Hilbert's transformation. In figure 3.19 it can be seen the time series of acceleration obtained by Semi-Analytical solution as well as its complete Hilbert's transformation and the window-filtered to 10m Hilbert's transformation. As it can be seen in the details of figure 3.19, the direct effect of it is the major demodulation of the signal. Moreover, the great advantage of this filter is that the integration of the Hilbert's transformation is limited to the set window of $10 \mathrm{~m}$ and not the whole domain of time as for the complete Hilbert's transformation. This fact points out the power of this filtered transformation which can obtain better results in a very shorted time of calculus. 


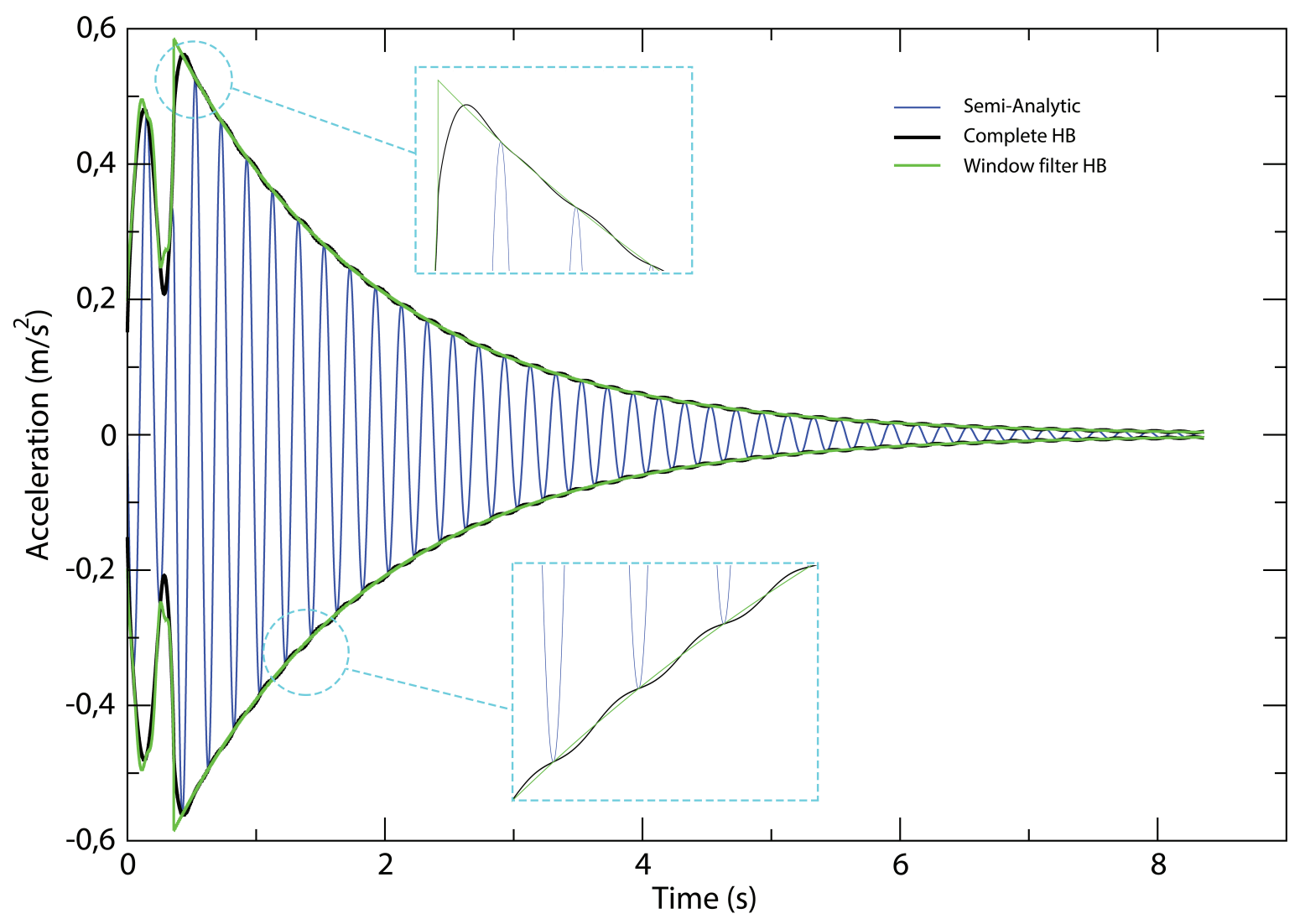

Figure 3.19: Acceleration time series of mid span of simply supported beam (IAPF) under a single load of $100 \mathrm{kN}$ at $150 \mathrm{~km} / \mathrm{h}$ obtained by Semi-Analytic solution, complete Hilbert transformation and Hilbert transformation with window filter of $10 \mathrm{~m}$.

\subsubsection{Effect of increasing time sampling}

The final aim of these transformations is the obtaining of the maximum envelope of response. Besides, as seen in latter sections, time series obtained by Hilbert's transformation are much smoother curves, what would allow to set higher time sampling for the collection of the maximum values with enough accuracy. Nevertheless, its obvious that a too high time stepping could not be able to collect all the peaks properly. Because of this, it results necessary to obtain ratios of applicability for different time sampling and structures. As the present research concerns, the three bidimensional structures analyzed in the $\mathrm{dq} / \mathrm{dq}$ method are once again analyzed with increasing time sampling of $T_{1} / 20, T_{1} / 10$ and $T_{1} / 20$ with window-filtered to 10m Hilbert's envelope. 


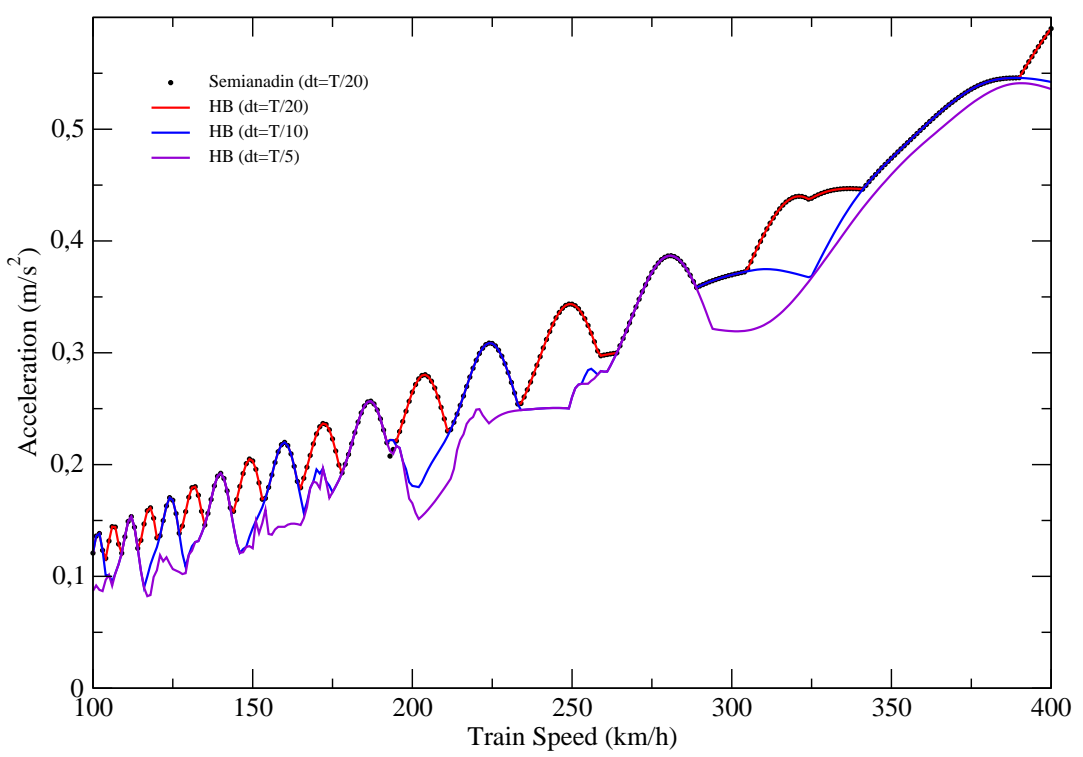

Figure 3.20: Acceleration envelope of mid central span of Henchi beam under a single load of $9.8 \mathrm{kN}$ by Semi-Analytic solution and Hilbert transformation for increasing time sampling

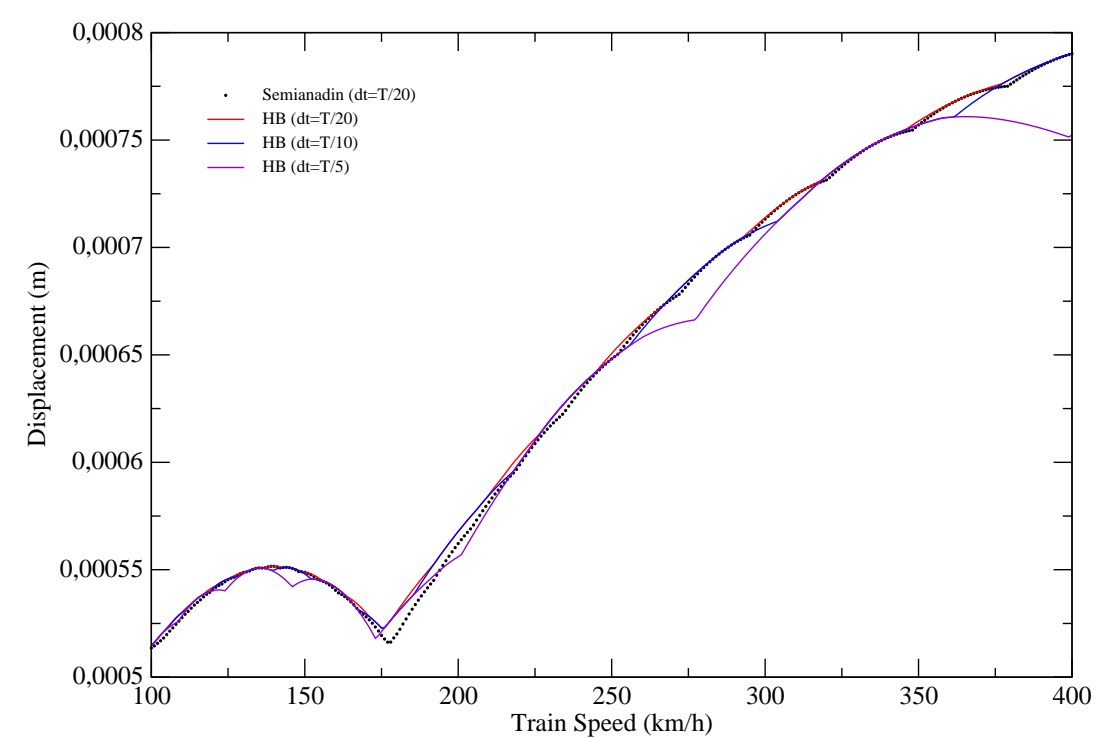

Figure 3.21: Displacement envelope of mid central span of Henchi beam under a single load of $9.8 \mathrm{kN}$ by Semi-Analytic solution and Hilbert transformation for increasing time sampling

In figure 3.21, it can be seen the limitations on the proposed methodology. Hilbert's envelope generates smoother time series which can be defined with higher time stepping. However, it can not be reduced too much because the signals would not be correctly sampled neglecting important peaks. This limitation explains why envelopes obtained by Hilbert's envelopes with time stepping of $T_{1} / 20$ has values under the one obtained by classic Semi-Analytic solution. This behavior is repeated in all the graphics. 


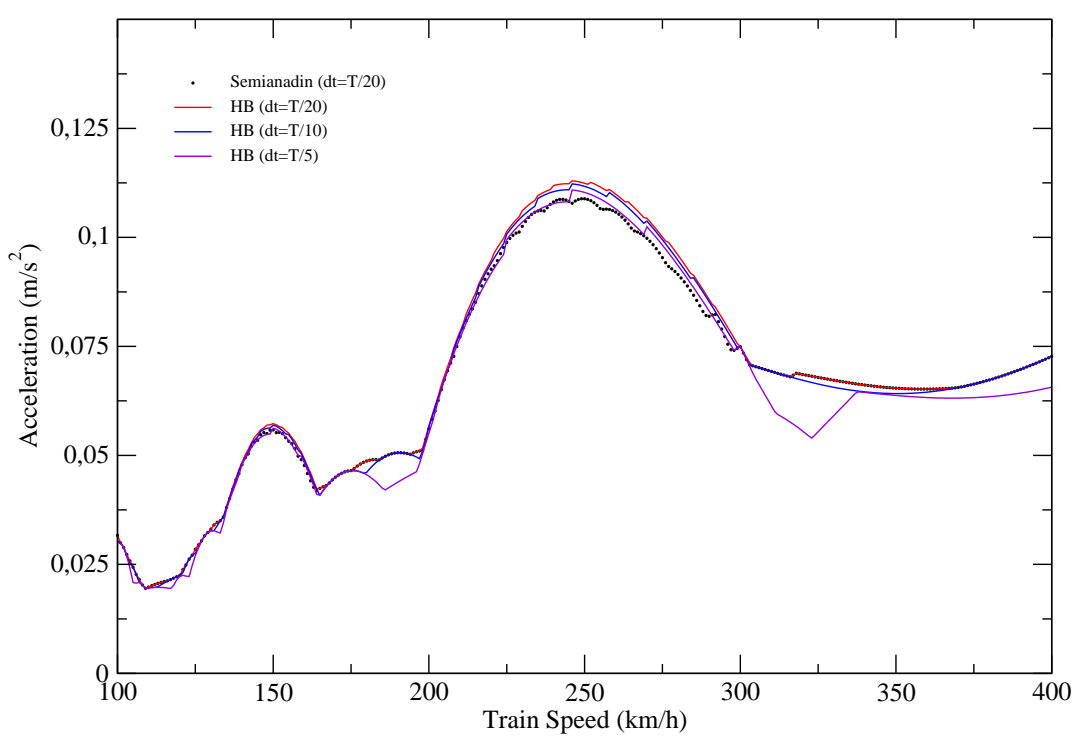

Figure 3.22: Acceleration envelope of mid span of Simply supported beam (IAPF) under a two loads of $9.8 \mathrm{kN}$ by Semi-Analytic solution and Hilbert transformation for increasing time sampling

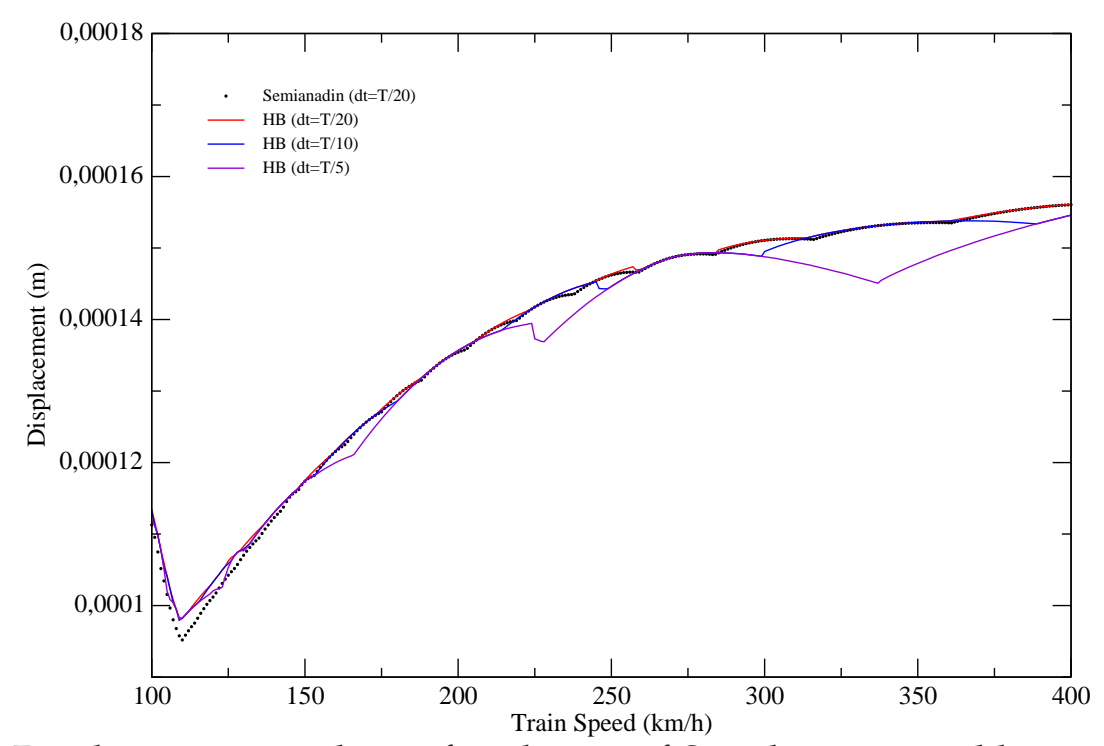

Figure 3.23: Displacement envelope of mid span of Simply supported beam (IAPF) under a two loads of $9.8 \mathrm{kN}$ by Semi-Analytic solution and Hilbert transformation for increasing time sampling 
Three-span bridge according to Eurocode 1.

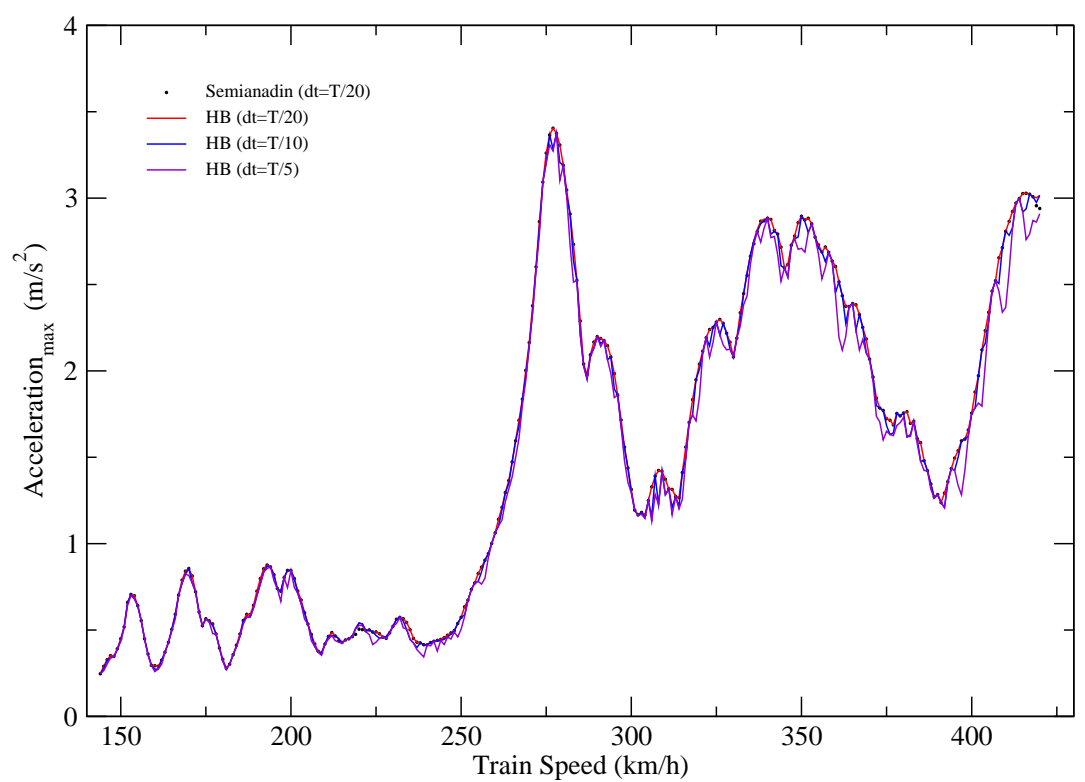

Figure 3.24: Acceleration envelope of mid span of Three-span bridge according to Eurocode 1 under the trains of HSML series by Semi-Analytic solution and Hilbert transformation for increasing time sampling

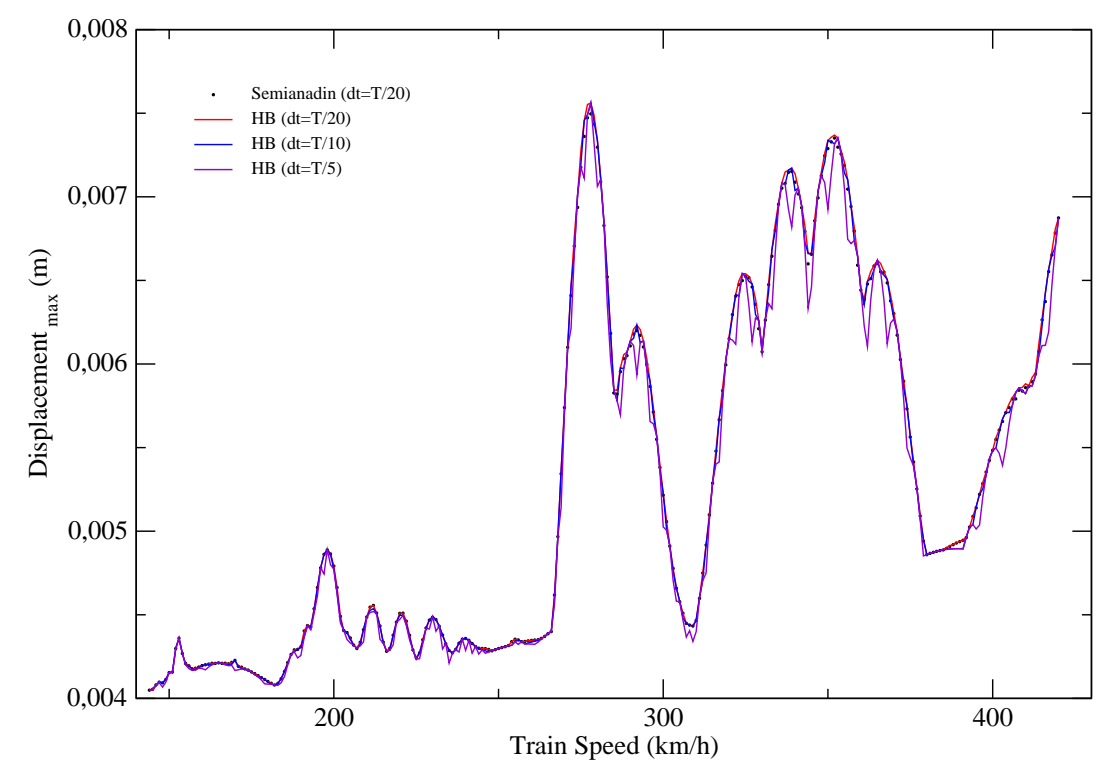

Figure 3.25: Displacement envelope of mid span of Three-span bridge according to Eurocode 1 under the trains of HSML series by Semi-Analytic solution and Hilbert transformation for increasing time sampling 


\section{Conclusion}

The envelopes of the dynamic response of structures obtained by window-filtered Hilbert's envelope can be defined with an equivalent accuracy to classical ones obtained by SemiAnalytical method but with higher time sampling. This fact is due to the demodulation of the signals provoked by Hilbert's envelope what generate smoother time series. Nevertheless, the fluctuating behavior of the response cannot be completely eliminated and it is more important the more complex is the scheme between load case and structure. Because of all this, as the time series are smoother, their definition can be obtained by shorter time stepping, although, it can't be too reduced because it would not be able to carry out the peaks' collection. In order to give some ratios of applicability, three bidimensional cases eere analyzed with increased time stepping, nevertheless, a more intensive research is needed in order to set rational ratios of time stepping in relation to the load cases and the forced structure.

The analysis took into consideration all the post-process points and trains, however, in this document only the results of the train A1 and the response in the mid-center span have been shown.

\subsubsection{Concrete box girder bridge. Rodenillo Bridge.}

The two following tests are tridimensional and correspond to the concrete box girder bridge and the steel concrete composite bridge analyzed in the previous method dq/dv. They two are analyzed with the proposed window-filtered Hilbert transformation method with increasing time sampling in order to observe the applicability of this new methodology. Some of the obtained results for this case can be seen in figures 3.26 and 3.27: 


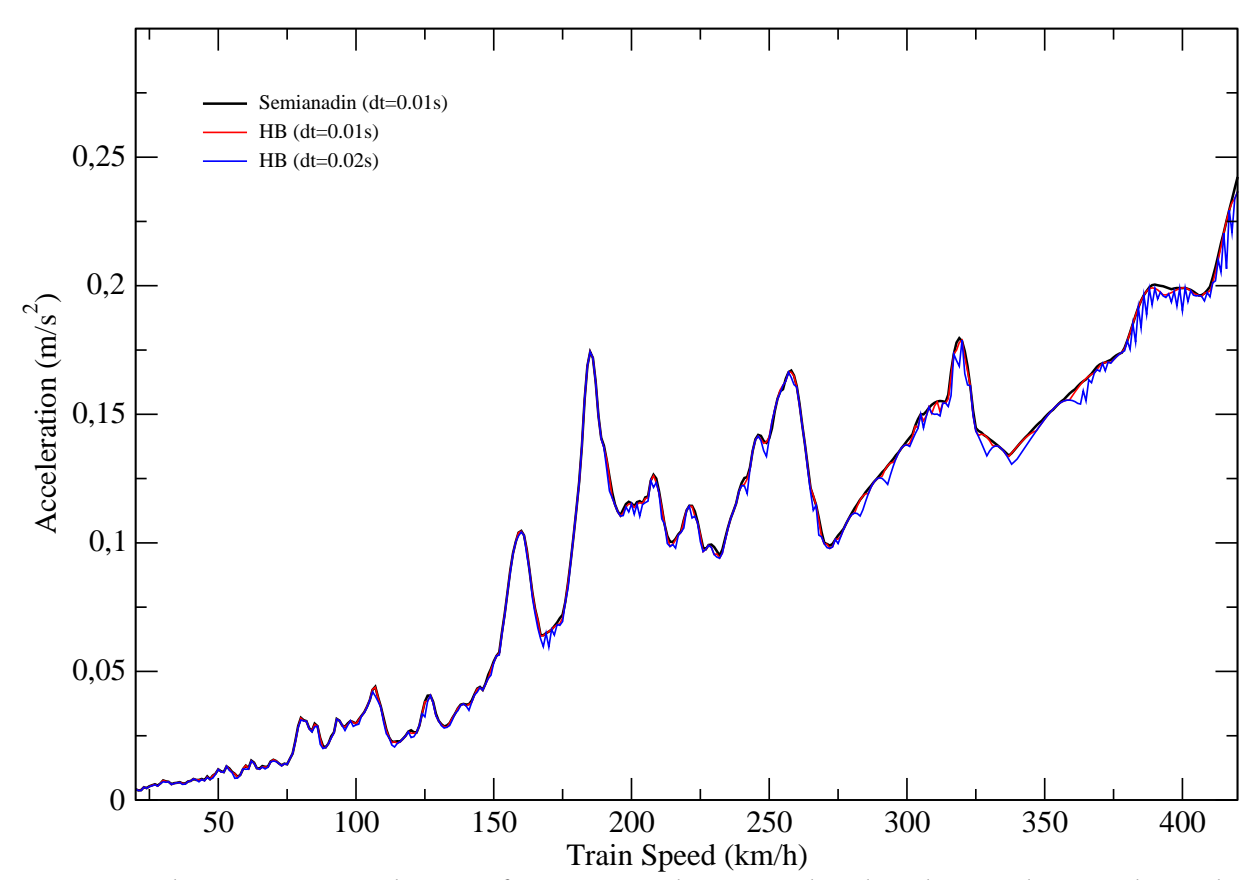

Figure 3.26: Acceleration envelope of concrete box girder bridge subjected to the passage of train A1 of HSML series by Semi-Analytic method and by $20 \mathrm{~m}$ window-filtered Hilbert envelope with increasing time sampling.

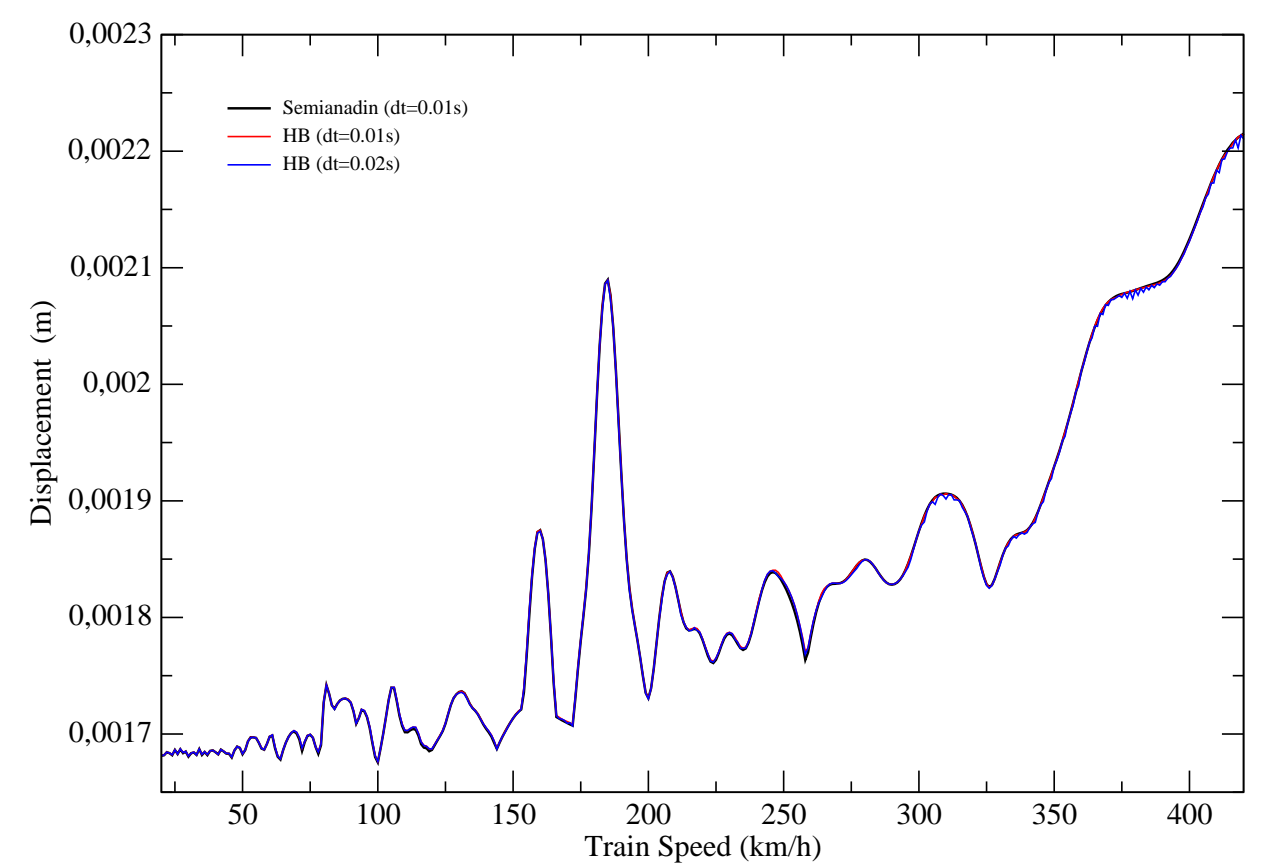

Figure 3.27: Displacement envelope of concrete box girder bridge subjected to the passage of train A1 of HSML series by Semi-Analytic method and by $20 \mathrm{~m}$ window-filtered Hilbert envelope with increasing time sampling.

\subsubsection{Steel-concrete composite bridge. Sesia viaduct.}

Finally, the same exactly process is followed in this last test. The results for the same train are exposed in 3.28 and 3.29: 


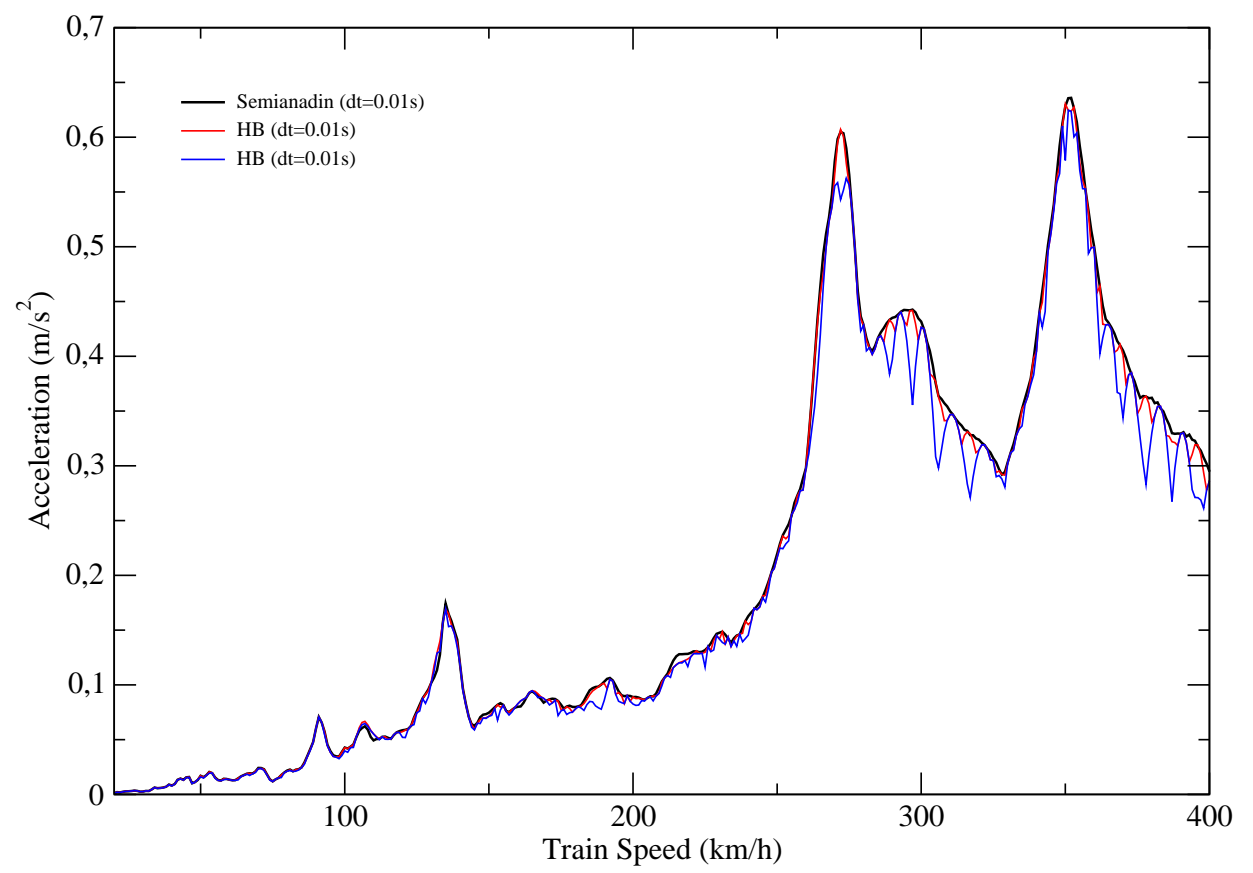

Figure 3.28: Acceleration envelope of steel-concrete composite bridge subjected to the passage of train A1 of HSML series by Semi-Analytic method and by 20m window-filtered Hilbert envelope with increasing time sampling.

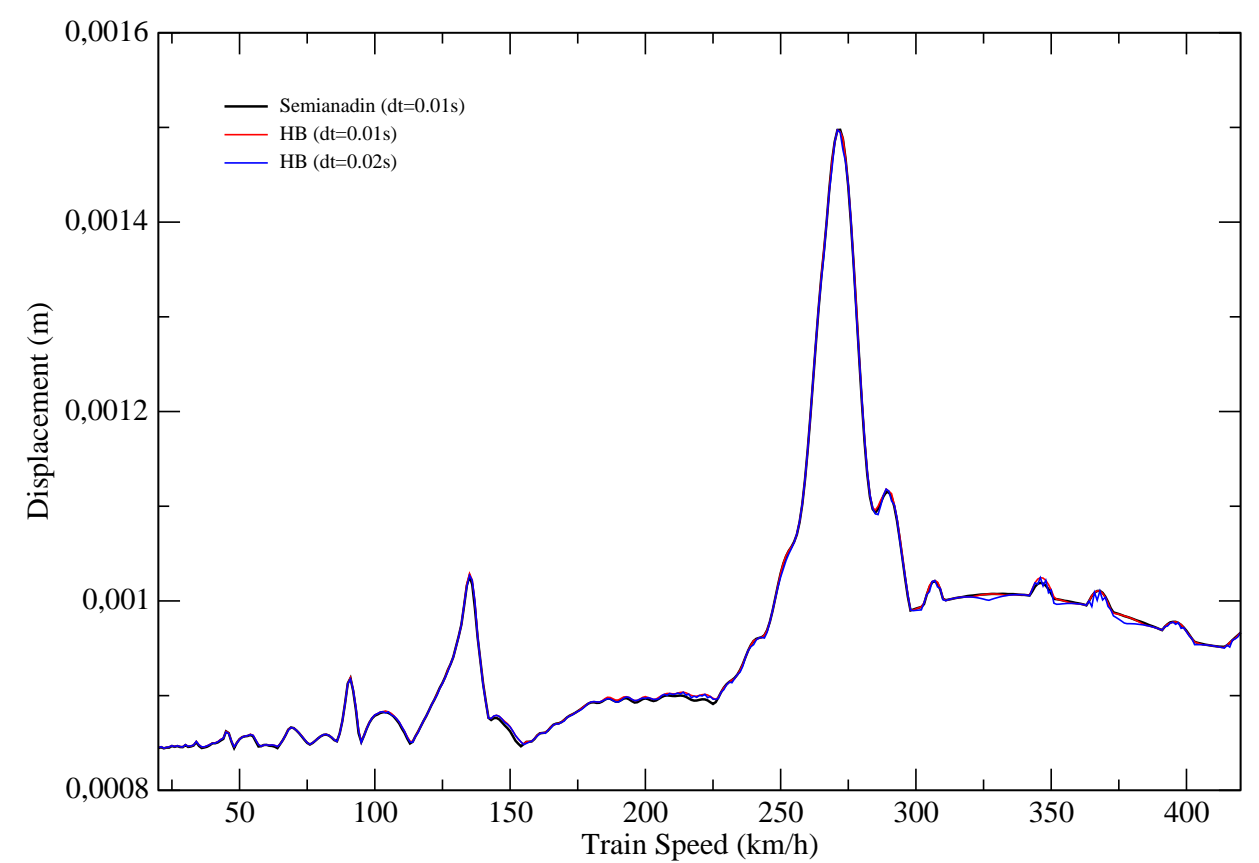

Figure 3.29: Displacement envelope of steel-concrete composite bridge subjected to the passage of train A1 of HSML series by Semi-Analytic method and by 20m window-filtered Hilbert envelope with increasing time sampling. 


\section{Conclusion}

In tridimensional structures is more remarkable the importance of defining minimum limits for time stepping. Specially, as seen in figures 3.26 and 3.28, excessive reduction in time sampling makes the method ignore non negligible peaks of the response. It is not aim of this present research, but in practical applications of this methodology a more rigorous investigation would be needed. 



\section{CHAPTER 4}

\section{Conclusions and future improvement}

\subsection{Conclusions}

In this research, two new methodologies of dynamic analysis of the response of structures subjected to moving load have been presented. On one hand, the dq/dq method obtains the response of the structure in terms of maximum values through the determination of the sensibility of the maximum response to velocity increments and a posterior cubic interpolation. On the other hand, a window-filtered Hilbert envelope method obtains the maximum envelope of the response through the analytical Hilbert transformation of the time series of response filtered to a fixed window in the time domain. This two new methodologies appears because of a need of reducing time of calculus and computational costs in dynamic analysis of pre-desing phases of structures. In this way, the two proposed method are thought to optimize the two main variables, velocity and time sampling respectively.

Several bidimensional and tridimensional test have been evaluated by this two methods in order to validate the results and to determine their applicability. The main conclusions are summarized below:

- Importance of refinement of boundary conditions in finite element models. Above all, this aspect is considerably relevant for $\mathrm{dq} / \mathrm{dv}$ method because of its direct proportional relationship with frequency. Sharp changes at endings of load line generates fictitious impulsive forces which excite the whole frequency spectrum, this fact enormously alter the sensitivity of the response, the more the higher derivative order of the response. A good solution of this problem has been seen to be the fixation of the vertical displacement and rotation at the cornes of the rail line.

- In the method $\mathrm{dq} / \mathrm{dv}$ the displacements are perfectly modeled unlike the response in terms of acceleration. This fact is due to the proportionality of the accelerations' sensitivity to train velocity in respect to the square value of frequency, unlike the sensitivity of displacements which are not directly proportional to frequency. This fact makes the solution in terms of acceleration be highly sensitive to fictitious excitement in the frequency domain because of a bad sampling of the series.

- Rayleigh's damping improves the fitting of the dq/dv methodology to the exact solution. This is due to the great mitigation of the contribution of high frequency modes imposed by this model, the ones whose disturbances more highly alter the sensitivity of the response to the train velocity. 
- $\mathrm{dq} / \mathrm{dv}$ method's fitting is not very accurate for low velocity for which the frequency spectrum is more distributed unlike for resonant velocities.

- Complete Hilbert transformation is not competitive to classical Semi-Analytic methods because it requires the integration of the signal along the whole time domain. Because of this, filters as window-filter must be applied in order to reduce time of calculus and computational costs.

- The Hilbert transformation of the homogeneous part of the Semi-Analytic solution is seen to be enough with the transformation of the separately trigonometric members.

- The proposed window filter applied to classic Hilbert transformation increases the demodulation of the response signals. Besides, the integration of the response is only carried out inside the window filter what considerably reduce the time of calculus.

- Hilbert transformation generates smoother time series what lets the setup of higher time stepping. However, the problem keeps on being a peak collection for which a minimum time stepping value has to de defined in order not to neglect important maximum values.

\subsection{Future improvement}

Two main lines of action for future research can be distinguished as follows:

1. Research on applicability of window-filtered Hilbert transform:

As it was mentioned in the last comment in the latter section, the application of Hilbert transformation generates smoother time series what allows the designer to set up higher time stepping. Nevertheless, it cannot be forgotten that the problem keeps on being a peak collection for which a minimum time stepping value must be defined so that not to neglect important maximum values. The definition of this limit values are not trivial because it depends on the fluctuating behavior of the response, what, a priori, seems to depend on the relationship between the structure and the characteristics of the structure. Besides, this determination also depends on the fixed window-filter what complicates its definition. Because of this, if this methodology wants to be applied for practical cases it should be previously researched the applicability of it in terms of time sampling and the length of the window filter.

2. Combination of the proposed two techniques:

This two methodologies optimize the dynamic analysis in two different and uncoupled ways. Because of this, it would be easy to combine them. In this way the scheme would be sequential: first of all the determination of the time series of the response would be carried out with a window-filtered Hilbert envelope method. This first phase tries to optimize the time of calculus in terms of time stepping. This process would be repeated for separated train velocities in order to finally adjust a cubic interpolation by the $\mathrm{dq} / \mathrm{dv}$ method. The derivative of the Hilbert transformation would be easy to obtain because it is still analytic in the time domain. This last stage would try to optimize the last variable, velocity stepping. A priori, it seems to be suitable the linkage 
of the two proposed method and it could suppose enormous reductions in time of calculus, fact that would result extremely interesting in pre-desing phases of structures subjected to dynamic actions. 



\section{Bibliography}

[1] P. Museros, A. Martínez-Castro, and A.Castillo-Linares. Design goes up a gear. Bridge. Design \& Engineering, Issue 33, Fourth Quarter:42-43, 2003.

[2] F. Bleich. Theorie und Berechnung der Eisernen Brücken. Springer, 1924.

[3] A. Kryloff. Über die erzwungenen schwingungen von gleichförmingen elastichen stäben. Mathematische Annalen, 1905.

[4] L. Frýba. Vibration of solids and structures under moving loads. 3rd ed. Thomas Telford, 1999.

[5] J. M. Biggs. Introduction to Structural Dynamics. Cambridge University Press, 1964.

[6] Y.B. Yang, J.D. Yau, and L.C. Hsu. Vibration of simple beams due to trains moving at high speeds. Engineering Structures, 19(11):936-944. doi:10.1016/S0141-0296(97)00001$1,1997$.

[7] Y.H. Chen and C.Y. Li. Dynamic response of elevated high-speed railway. Journal of Bridge Engineering, 5:124-130, 2000.

[8] T. Hayashikawa and N. Watanabe. Dynamic behavior of continuous beams with moving loads. Journal of the Engineering Mechanics Division, ASCE, 107:229-246, 1981.

[9] R.W.Clough and J.Penzien. Dynamics of Structures. Mac Graw-Hill, 1993.

[10] N. M. Newmark. A method of computation for structural dynamics. ASCE, 1959.

[11] R.Calcada, A.Cunha, and R.Delgado. Dynamic analysis of metallic arch railway bridge. Bridge Engineering., 7:214-222, 2002.

[12] Y.S. Wu, Y.B. Yang, and J.D.Yau. Three-dimensional analysis of train-rail-bridge interaction problems. Vehicle System Dynamics, 36(1):1-35, 2001.

[13] J.W. Kwark, E.S.Choi, Y.J.Kim, B.S.Kim, and S.I.Kim. Dynamic behavior of two-span continuous concrete bridges under moving high-speed train. Computer and Structures., 82:463-474, 2004.

[14] R. Karoumi. Response of cable-stayed and suspension bridges to moving vehicles. Analysis methods and practical modelling techniques. PhD thesis, Department of Structural Engineering, KTH Royal Institute of Technology, Stockholm, Sweden, 1998.

[15] M. Majka and M. Hartnett. Effects of speed, load and damping on the dynamic response of railway bridges and vehicles. Computer and Structures, 86:556-572, 2008.

[16] H. Xia, N. Zhang, and G. D. Roeck. Dynamic analysis of high speed railway bridge under articulated trains. Computers and Structures, 81:2467-2478, 2003.

[17] Y. S. Cheng, F. T. K. Au, and Y. K. Cheung. Vibration of railway bridges under a moving train by using bridgetrack-vehicle element. Engineering Structures, 23:1597-1606, 2001. 
[18] Instrucción de acciones a considerar en el proyecto de puentes de ferrocarril (IAPF), 2007.

[19] Y. Dugush and M. Eisenberger. Vibrations of non-uniform continuous beams under moving loads. Sound and Vibration., 254:911-926, 2002.

[20] P. Museros, A. Martínez-Castro, and A.Castillo-Linares. Semi-analytic solution in the time domain for non-uniform multi-span bernoulli-euler beams traversed by moving loads. Journal of Sound and Vibration, 294:278-297, 2006.

[21] P. Museros, A. Martínez-Castro, and A.Castillo-Linares. Solución semianalítica al problema de paso de trenes de alta velocidad sobre puentes hiperestáticos de sección variable. Actas del VI Congreso de Métodos Numéricos en Ingeniería, Lisboa, 2004.

[22] P. Museros, A. Martínez-Castro, and A.Castillo-Linares. Semi-analytic solution for nonuniform euler-bernoulli beams under moving forces. Proceedings of the 7 th International Conference on Computational Structures Technology, Lisboa, 294:278-297, 2004.

[23] A.Castillo-Linares, M.Villameriel-Fernández, G. Montero-Poyatos, M.García-Ramírez, J.de Dios-Moreno-Jiménez, and A.Martínez-Castro. Puente arco sobre la línea de alta velocidad córdoba-málaga. condicionantes dinámicos y constructivos para el diseño. Hormigón y acero, 258, 2010.

[24] Gabor. Theory of communication. Journal of Institute of Electrical Engineers, 93:429-457, 1946.

[25] E. Bedrosian. A product theorem for Hilbert transform., 1963.

[26] M. Feldman. Hilbert transform in vibration analysis. Mechanical Systems and Signal Processing, 25:(735-802), 2010.

[27] M. Simon and G. R. Tomlinson. Use of the hilbert transform in modal analysis of linear and non-linear structures. Journal of Sound and Vibration, 4:421-436, 1984.

[28] G. R. Tomlinson. Developments in the use of the hilbert transform for detecting and quantifying non-linearity associated with frequency response functions. Mechanical Systems and Signal Processing, 2:151-171, 1987.

[29] M. Feldman. Non-linear system vibration analysis usign hilbert transform-free vibration analysis method freevib. Mechanical Systems and Signal Processing, 2:119-127, 1994.

[30] N. E. Huan G and Z. Shen and R. L. Steven and W. C. Manli and H. S. Hsing and A. Quanan and Y. Nai-Chyuan and C. T. Chi and H. L. Henry. The empirical mode decomposition and the Hilbert spectrum for nonlinear and non-stationary time series analysis, 1998.

[31] P-W. Tseb Z. K. Penga and F. L. Chua. An improved hilbert-huang transdorm and its application in vibration signal analysis. Journal of Sound and Vibration, 286:187-205, 2005.

[32] R. R. Zhang and L. D. Olson. Dynamic bridge substructure condition assessment with hilbert -huang transform: Simulated flood earthquake damage to monitor structural health and security. Transportation Research Record: Journal of the Transportation Research Board., pages 153-159, 2007. 
[33] Z. K. Penga, P. W. Tsea, and F. L. Chub. Hilbert-huang transform and wavelet transfom: application to fault diagnosis for rolling bearing. Mechanical Systems and Signal Processing, 19:974-988, 2005.

[34] D. Yinfenga, L. Yingmina, X. Mingkuia, and L. Mingb. Analysis of earthquake ground motions using an improved hilbert-huang transform. Soil Dynamics and Earthquake Engineering, 28:7-19, 2008.

[35] C. Baoa, H. Hao, Z. X. Li, and X. Zhub. Time-varying system identification using a newly improved hht algorithm. Computer and Structures, 87:1611-1623, 2009.

[36] T.M. Apostol. Mathematical Analysis. Adisson Wesley, 1967.

[37] R. G. Bartle. The Elements of Real Analysis. John Milley, 1976.

[38] R.C. Buck. Advanced Calculus. 1978.

[39] J. de Burgos. Cálculo infinitisimal de varias variables. 1995.

[40] G. Brandley and K. Smith. Cálculo de varias variables.

[41] J. S. Canovas and R. Finney. Fundamentos matemáticos de la ingeniería.

[42] J. J. Duistermaat and J. A. C. Kolk. Multidimensional Real Analysis. Cambridge University Press, 2004.

[43] M. Krasnov, A.Kiseliov, G. Makarenko, and E. Shikin. Curso de matemáticas superiores para ingenieros. 1994.

[44] K. Henchi, M. Fafard, G. Dhatt, and M. Talbot. Dynamic behaviour of multispan beams under moving loads. Journal of Sound and Vibration, 199(1):33-50. doi:10.1006/jsvi.1996.0628, 1997.

[45] E. G. Macías. Dinámica de puentes arco-atirantados mixtos para el ferrocarril de alta velocidad. estudio de un caso, planteamiento de alternativas y conclusiones para el diseño. Master's thesis, University of Granada, Spain, 2012.

[46] H. Zhou, K. Liu, Y. Q. Wang, Shi Y. J, and G. De Roeck. Fatigue assesment of a composite railway bridge for high speed trains. part i: Modeling and fatigue critical details. Journal of Constructional Steel Research, 82:234-245, 2013.

[47] W. W. Guo, H. Xia, G. De Roeck, and K. Liu. Integral model for train-track-bridge interaction on the sesia viaduct: Dynamic simulation and critical assesment. Computers and Structures, 112-113:205-216, 2012.

[48] K. Liu, E. Reynders, G. De Roeck, and G. Lombaert. Experimental and numerical analysis of a composite bridge for high-speed trains. Journal of Sound and Vibration, 320:201220, 2008.

[49] K. Liu, H. Zhou, G. Shi, Y. Q. Wang, Y. J. Shi, and G. De Roeck. Fatigue assesment of a composite railway bridge for high speed trains. part ii: Conditions fo which a dynamic analysis is needed. Journal of Constructional Steel Research, 82:246-254, 2013.

[50] S. L. Hahn. Signals, Oscillarions and Waves. Artech House, 1996.

[51] M. Abramowitz and I. A. Stegun. Handbook of Mathematical Functions with Formulas, Graphs, and Mathematical Tables. 1972. 